\title{
ALPHA-CLUSTER PICKUP FROM Te AND Sn ISOTOPES WITH THE (d, $\left.{ }^{6} \mathrm{Li}\right)$ REACTION
}

\author{
J. JÄNECKE and F. D. BECCHETTI ${ }^{\dagger}$
}

Department of Physics, The University of Michigan, Ann Arbor, Michigan 48109

and

\section{E. THORN}

Department of Physics, Brookhaven National Laboratory, Upton, LI, New York $11973^{\text {t* }}$

Received 26 February 1979

\begin{abstract}
The reactions ${ }^{122,124,126,128,130} \mathrm{Te}\left(\mathrm{d},{ }^{6} \mathrm{Li}\right)^{118,120,122,124,126} \mathrm{Sn}$ and ${ }^{112,116,118,120,122,124 .}$ ${ }^{126} \mathrm{Sn}\left(\mathrm{d},{ }^{6} \mathrm{Li}\right){ }^{108.112 .114 .116 .118 .120} \mathrm{Cd}$ have been investigated at $E_{\mathrm{d}}=33 \mathrm{MeV}$ for states up to $E_{\mathrm{x}}=3 \mathrm{MeV}$ or higher using magnetic analysis. Alpha-particle spectroscopic factors and reduced $\alpha$-widths have been extracted with zero- and finite-range distorted wave Born approximation (DWBA) theory. Spectroscopic amplitudes were calculated from shell-model configurations including core excitations and a semi-microscopic analysis was performed for ground state transitions, proton pairing vibration states, as well as certain states with $J^{\star} \neq 0^{+}$. Good agreement is obtained for the transitions to the Cd ground states provided DWBA is normalized to $\alpha$-decay. The ground states and proton pairing vibration states in the heavy $\mathrm{Sn}$ isotopes appear to be strongly mixed. Increased collectivity, predicted by the interacting boson model, affects the excitation energies and transition strengths of the $0^{+}$proton pairing vibration states in $\mathrm{Sn}$ in the middle of the neutron shell. Selectivity and coherence phenomena are also prevalent for states with $J^{\boldsymbol{x}} \neq 0^{+}$and are interpreted microscopically. In particular, coherent contributions from proton pair and neutron pair excitations lead to enhancements not observed in two-nucleon transfer. The mass excess of ${ }^{120} \mathrm{Cd}$ was measured as $-83975 \pm 25 \mathrm{keV}$. About 25 previously unknown states were observed in the $\mathrm{Sn}$ and $\mathrm{Cd}$ isotopes and about 30 spin-parity assignments were made.
\end{abstract}

NUCLEAR REACTIONS ${ }^{122.124 .126 .128 .130} \mathrm{Te},{ }^{112.116 .118 .120 .122 .124} \mathrm{Sn}\left(\mathrm{d},{ }^{6} \mathrm{Li}\right)$, $E=33 \mathrm{MeV}$; measured $\sigma\left(E_{\mathrm{o}_{\mathrm{L},}}, \theta\right) .{ }^{118.120 .122,124,126} \mathrm{Sn}, 108,112,114,116,118,120 \mathrm{Cd}$

E deduced levels, $J^{\pi}, S_{x}, \gamma_{2}^{2}$. Measured $Q\left({ }^{124} \mathrm{Sn}\left(\mathrm{d},{ }^{6} \mathrm{Li}\right)^{120} \mathrm{Cd}=-5216 \pm 24 \mathrm{keV}, \Delta M\left({ }^{120} \mathrm{Cd}\right)\right.$ $=-83975 \pm 25 \mathrm{keV}$. DWBA analyses. Semi-microscopic analyses with shell model spectroscopic amplitudes.

\section{Introduction}

Correlations between nuclear wave functions reflect upon the existence of substructures in nuclei. Of these, neutron pairing correlations are by far the best understood correlations as they have been studied extensively throughout all regions of nuclei

+ Supported in part by the National Science Foundation under Grant PHY78-07754.

${ }^{\dagger+}$ Supported by USDOE. 
by means of two-neutron transfer reactions, most notably the $(p, t)$ and $(t, p)$ reactions. These reactions display a remarkable degree of selectivity. Ground states of even-even nuclei are usually strongly excited as are certain excited $0^{+}$states. The pairing vibrational model ${ }^{1,2}$ ) accounts for many of the observed phenomena. The microscopic description of two-neutron transfer reactions constitutes a sensitive probe of nuclear wave functions since coherent sums have to be taken over shell-model configurations as well as over different numbers of harmonic oscillator quanta required to describe the center-of-mass (c.m.) motion of the transferred pair.

Proton pairing correlations have been studied less extensively. This is at least partly due to experimental factors. The $\left({ }^{3} \mathrm{He}, \mathrm{n}\right)$ stripping reaction, for example, requires neutron time-of-flight spectroscopy, and the energy resolution, particularly at higher bombarding energies is much worse than that of charged-particle reactions. Heavy-ion reactions transferring two protons have been studied, but they often lead to rather structureless angular distributions and the analysis is complicated.

Recent experimental ${ }^{3-7}$ ) and theoretical ${ }^{8-13}$ ) investigations suggest a close relationship between $\alpha$-cluster and two-nucleon transfer reactions. Kurath and Towner ${ }^{9}$ ) have shown that $\alpha$-particle spectroscopic amplitudes involve a coherent sum of coupled two-neutron and two-proton spectroscopic amplitudes, with the neutrons and protons in singlet-even states $(S=0, T=1)$. Selectivity and coherence phenomena known in two-nucleon transfer are therefore expected to be even more enhanced in $\alpha$-transfer. This result also suggests that the existence of strong pairing correlations in nuclei could be responsible for a much simpler description and interpretation of $\alpha$-transfer processes than one might naively expect. While a strong correspondence between $\alpha$-transfer and these two-nucleon transfers has indeed been observed experimentally, even for odd- $A$ targets ${ }^{4}$ ), more experimental information is needed to better understand and subsequently exploit the phenomena inherent in $\alpha$-cluster transfers.

Reactions involving the transfer of a neutron-proton pair such as $(\alpha, d),(d, \alpha)$, $\left({ }^{3} \mathrm{He}, \mathrm{p}\right)$ and $\left(\mathrm{p},{ }^{3} \mathrm{He}\right)$ display selectivity and coherence phenomena but cross sections are generally smaller than for two-neutron and two-proton transfer. No obvious relation between $\alpha$-transfer and np transfer has been observed so far, but it is conceivable that such connections exist for transitions to certain high-spin states.

It is worth noting that $\alpha$-cluster transfer reactions are intimitely linked to $\alpha$-decay as both yield $\alpha$-particle reduced widths $\gamma_{\alpha}^{2}$. Thus, the comparison between $\alpha$-cluster pickup on radioactive targets [e.g. ${ }^{144} \mathrm{Nd},{ }^{148} \mathrm{Sm},{ }^{152} \mathrm{Gd}$, refs. ${ }^{6,14}$ ), or ${ }^{232} \mathrm{Th},{ }^{238} \mathrm{U}$, ref. $\left.\left.{ }^{15}\right)\right]$ and $\alpha$-decay provides a test for DWBA calculations and their sensitivity to wave functions, optical-model parameters, and so on. A reanalysis of the reaction ${ }^{148} \mathrm{Sm}\left(\mathrm{d},{ }^{6} \mathrm{Li}\right){ }^{144} \mathrm{Nd}$ studied earlier $\left.{ }^{6}\right)$ is included in the present work to permit the extraction of absolute spectroscopic information.

The $\alpha$-cluster pickup reactions Te(d, $\left.{ }^{6} \mathrm{Li}\right) \mathrm{Sn}$ and $\mathrm{Sn}\left(\mathrm{d},{ }^{6} \mathrm{Li}\right) \mathrm{Cd}$ on most even- $A$ targets were chosen for a systematic experimental investigation as the range of available isotopes is very broad and allows one to study the dependence on neutron 
excess. Also, an experimental study of the $(p, t)$ reaction on the even- $A$ Sn-isotopes has been performed together with a microscopic analysis $\left.{ }^{16}\right)$. In addition the $\left({ }^{3} \mathrm{He}, \mathrm{n}\right)$ reaction on $\mathrm{Cd}$ and $\mathrm{Sn}$ targets has recently been investigated ${ }^{17}$ ) with the observation of strong transitions to ground and certain excited $0^{+}$states. Theoretical BCS wave functions are available for nuclei in the $\mathrm{Sn}$ region ${ }^{18,19}$ ) and wave functions for the ground states of the even- $A \mathrm{Te}$ and $\mathrm{Cd}$ isotopes have also become available ${ }^{13,20}$ ). A unified description of collective nuclear states in terms of a system of interacting bosons has been introduced by Arima and Iachello ${ }^{21-24}$ ). The model appears to describe vibrational ${ }^{22}$ ), rotational ${ }^{23}$ ), and transitional ${ }^{24}$ ) nuclei quite well and one might expect that the properties of certain excited states in $\mathrm{Sn}$ and nearby isotopes can be described in the framework of this model.

The experimental procedures and results are presented in sects. 2 and 3 . The systematics of excitation energies is discussed in sect. 4. Various aspects of DWBA analyses for $\left(\mathrm{d},{ }^{6} \mathrm{Li}\right)$ are outlined in sect. 5. The results are discussed in sect. 6. A short summary is presented in sect. 7. Details of the microscopic analysis are included in the appendices. Earlier accounts of this work have been reported elsewhere ${ }^{25}$ ).

\section{Experimental procedures}

Spectra and angular distributions for ${ }^{6} \mathrm{Li}$ particles from the $\left(\mathrm{d},{ }^{6} \mathrm{Li}\right)$ reaction on Te and $\mathrm{Sn}$ isotopes were measured utilizing a $33 \mathrm{MeV}$ deuteron beam from the Brookhaven National Laboratory double tandem Van de Graaf facility. A sputter ion source employing a deuterated titanium cone provided beams of 200 to $300 \mathrm{nA}$ on target. Targets consisted of highly enriched Te and Sn metal 100 to $280 \mu \mathrm{g} / \mathrm{cm}^{2}$ thick evaporated onto carbon backings of $20 \mu \mathrm{g} / \mathrm{cm}^{2}$ thickness. The target thicknesses were obtained from the comparison of forward-angle deuteron elastic scattering with optical-model predictions as well as the energy loss of $5.5 \mathrm{MeV} \alpha$-particles. Unfortunately, burn spots developed on some of the Te targets due to the sharply focused deuteron beam. The beam was therefore slightly defocused during the later runs. Absolute cross sections are considered to be accurate to within $\pm 30 \%$. The energy loss of the outgoing ${ }^{6} \mathrm{Li}$ particles in the target was the major contribution to the energy resolution of 35 to $80 \mathrm{keV}$ FWHM. The ${ }^{6} \mathrm{Li}$ particles were detected and identified with a position-sensitive proportional counter detection system in the focal plane of the BNL QDDD magnetic spectrograph. The acceptance angle of the spectrometer was set at $\pm 3^{\circ}$ providing a solid angle of $10 \mathrm{msr}$. The effective length of the detector was $65 \mathrm{~cm}$ which covered a range of excitation energies of about $3 \mathrm{MeV}$. A monitor consisting of a small plastic scintillator and a photoelectron multiplier was used during most runs. 


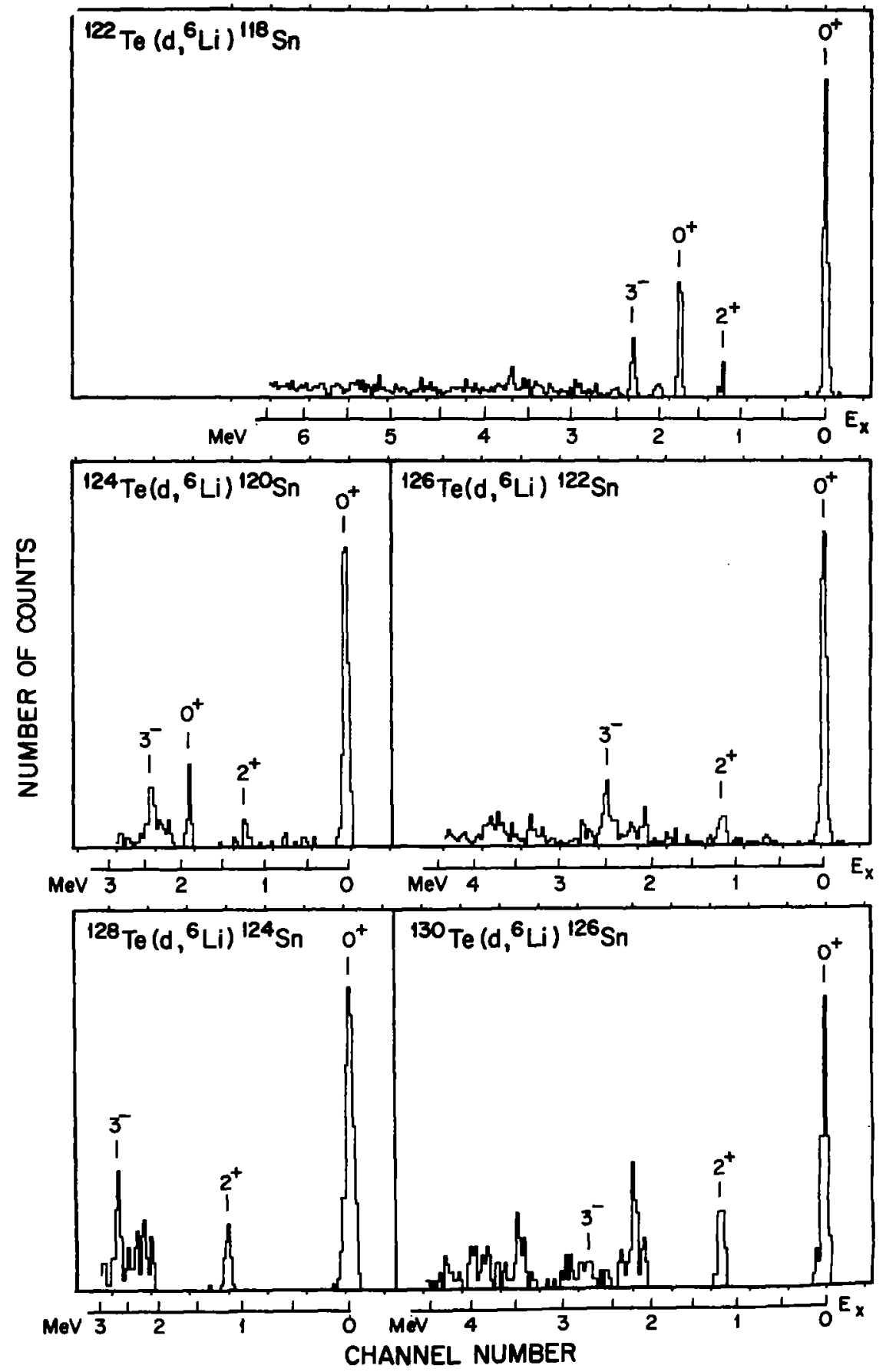

Fig. 1. Energy spectra for ${ }^{6} \mathrm{Li}$ particles from the Te(d, $\left.{ }^{6} \mathrm{Li}\right) \mathrm{Sn}$ reactions obtained at $E_{\mathrm{d}}=33 \mathrm{MeV}$ and $\theta_{\text {lab }}=16^{\circ}$. 


\section{Experimental results}

\subsection{ENERGY SPECTRA}

Fig. 1 shows ${ }^{6} \mathrm{Li}$ energy spectra obtained for the even- $A$ Te targets at $\theta_{1 \mathrm{ab}}=16^{\circ}$. Similarly, fig. 2 shows the results for the even- $A$ Sn targets. Only ${ }^{120} \mathrm{Te}$ and ${ }^{114} \mathrm{Sn}$ were not included in the investigation because of their low natural isotopic abundances of $0.09 \%$ and $0.66 \%$, respectively. Most spectra cover the region up to $E_{\mathrm{x}} \approx 3 \mathrm{MeV}$ and several extend beyond. The latter are the result of two or three overlapping exposures. The spectra in figs. 1 and 2 have been arbitrarily normalized to display approximately the same ground-state peak heights. All spectra are dominated by the strong $0^{+}$ground-state transitions. Low excited $0^{+}$states in ${ }^{118} \mathrm{Sn}$ and ${ }^{120} \mathrm{Sn}$ are also populated strongly, particularly in ${ }^{118} \mathrm{Sn}$ where the cross section is about $45 \%$ that of the ground state (g.s.). This state is also excited strongly in the $\left({ }^{3} \mathrm{He}, \mathrm{n}\right)$ reaction $\left.{ }^{17}\right)$ where it has been identified as a state which carries a large fraction of the proton pairing vibration strength. It is only weakly excited in the $(p, t)$ reaction $\left.{ }^{16}\right)$. Other states up to about $3 \mathrm{MeV}$ excitation energy are excited with up to $60 \%$ of the g.s. strength, particularly the $3^{-}$states at $E_{\mathrm{x}} \approx 2.0-2.5 \mathrm{MeV}$ and the $2^{+}$states at $E_{\mathrm{x}} \approx 1.2 \mathrm{MeV}$ in $\mathrm{Sn}$ and $E_{\mathrm{x}} \approx 0.6 \mathrm{MeV}$ in Cd. Very little strength is observed for transitions to states above $E_{\mathrm{x}} \approx 3 \mathrm{MeV}$. Interference from the much more intense $\left(\mathrm{d},{ }^{6} \mathrm{Li}\right)$ reactions on the carbon backing or contaminations presented no problem because of the much more negative $Q$-values for the latter. Only for the reaction with the most negative $Q$-value, ${ }^{124} \mathrm{Sn}\left(\mathrm{d},{ }^{6} \mathrm{Li}\right){ }^{120} \mathrm{Cd}$, was the ${ }^{16} \mathrm{O}\left(\mathrm{d},{ }^{6} \mathrm{Li}\right){ }^{12} \mathrm{C}_{\text {g.s. }}$. transition observed at higher excitation energy (see fig. 2).

It is interesting to note the basic differences between the spectra observed in $\alpha$ cluster and two-neutron pickup. The spectra measured in a systematic study of the $\mathrm{Sn}(\mathrm{p}, \mathrm{t}) \mathrm{Sn}$ reactions at $E_{\mathrm{p}}=20 \mathrm{MeV}$ on even- $A$ Sn targets ${ }^{16}$ ) show very strong g.s. transitions with cross sections 3 to 30 times that for the formation of $2^{+}$and $3^{-}$states. The results from other $(\mathrm{p}, \mathrm{t})$ and $(\mathrm{t}, \mathrm{p})$ investigations on even- $A$ Sn targets are very similar ${ }^{26-29}$ ). However, contrary to $\left(d,{ }^{6} \mathrm{Li}\right)$ (see figs. 1 and 2 ), transitions to excited states are much weaker in $(\mathrm{p}, \mathrm{t})$. Most cross sections are less than $10 \%$ of the g.s. cross sections, and only the transitions to the first excited $2^{+}$states in the heavy $\mathrm{Sn}$ isotopes exceed $20 \%$. Transitions to excited $0^{+}$states are characteristically the weakest in $(p, t)$ attaining, at most, $3 \%$ of the g.s. strength. In contrast, the $\left(d,{ }^{6} \mathrm{Li}\right)$ transition to the $0^{+}$state in ${ }^{118} \mathrm{Sn}$ at $E_{\mathrm{x}}=1758 \mathrm{keV}$ has a cross section of about $45 \%$ of the g.s. transition. It will be shown below that the differences between $(p, t)$ and $\left(\mathrm{d},{ }^{6} \mathrm{Li}\right)$ are due to a combination of kinematic and spectroscopic conditions. It is worth noting, though, that the $(p, t)$ cross sections for the ground state transitions are almost 3 orders of magnitude greater (first $l=0$ maximum outside $0^{\circ}$ ) than the corresponding $\left(\mathrm{d},{ }^{6} \mathrm{Li}\right)$ cross sections.

Angular distributions from $\theta_{1 \mathrm{lab}}=5^{\circ}$ to $61^{\circ}$ were measured for ${ }^{122} \mathrm{Te}\left(\mathrm{d},{ }^{6} \mathrm{Li}\right){ }^{118} \mathrm{Sn}$. They are displayed in figs. 3 and 4 along with DWBA curves. These will be discussed later. More limited data, namely spectra at one or two angles, were obtained for the 

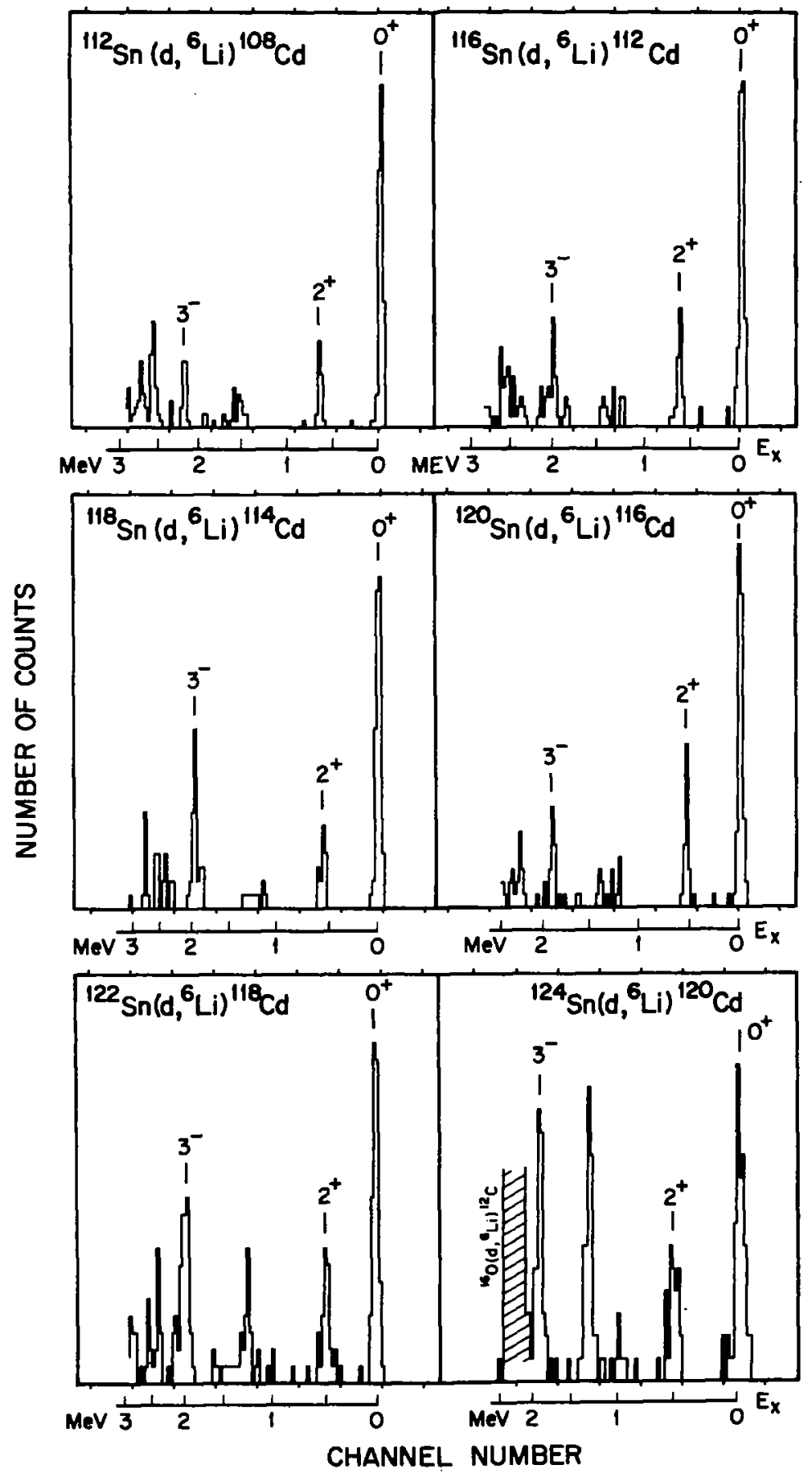

Fig. 2. Energy spectra for ${ }^{6} \mathrm{Li}$ particles from the $\mathrm{Sn}\left(\mathrm{d},{ }^{6} \mathrm{Li}\right) \mathrm{Cd}$ reactions obtained at $E_{\mathrm{d}}=33 \mathrm{MeV}$ and $\theta_{\text {leb }}=16^{\circ}$. 
other targets. Earlier data including angular distributions for even- $A$ Sn targets as well as ${ }^{117} \mathrm{Sn}$ and ${ }^{119} \mathrm{Sn}$ have been presented elsewhere ${ }^{3,4}$ ).
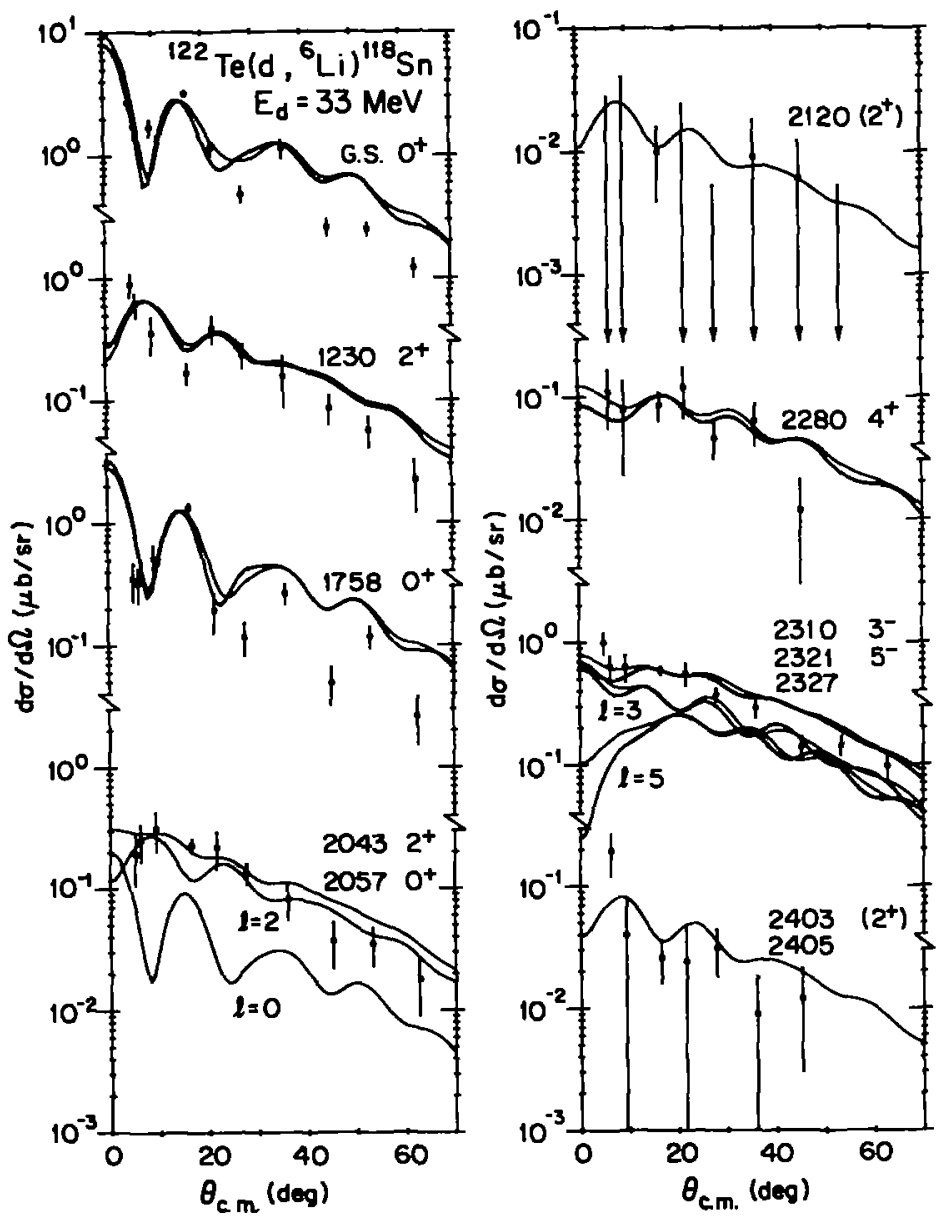

Fig. 3. Experimental and calculated angular distributions for transitions to states in ${ }^{118} \mathrm{Sn}$ from the ${ }^{122} \mathrm{Te}\left(\mathrm{d},{ }^{6} \mathrm{Li}\right){ }^{118} \mathrm{Sn}$ reaction at $E_{\mathrm{d}}=33 \mathrm{MeV}$. The curves are based on zero-range (thin lines) and finiterange (thick lines) DWBA calculations. They are averaged over $\Delta \theta= \pm 3^{\circ}$ to account for the experimental angular resolution.

\subsection{THE Te(d, $\left.{ }^{6} \mathrm{Li}\right) \operatorname{Sn}$ REACTIONS}

Tables 1 to 5 list for all Te targets the measured c.m. differential cross sections at $\theta_{\text {lab }}=16^{\circ}$ for states in the final Sn nuclei. Some of the observed transitions could not be resolved. Relative contributions are estimated wherever possible. These were obtained from the decomposition of the angular distributions $\left({ }^{122} \mathrm{Te}\right.$ target only) in conjunction with a careful study of the systematics of the $N$-dependence of cross 

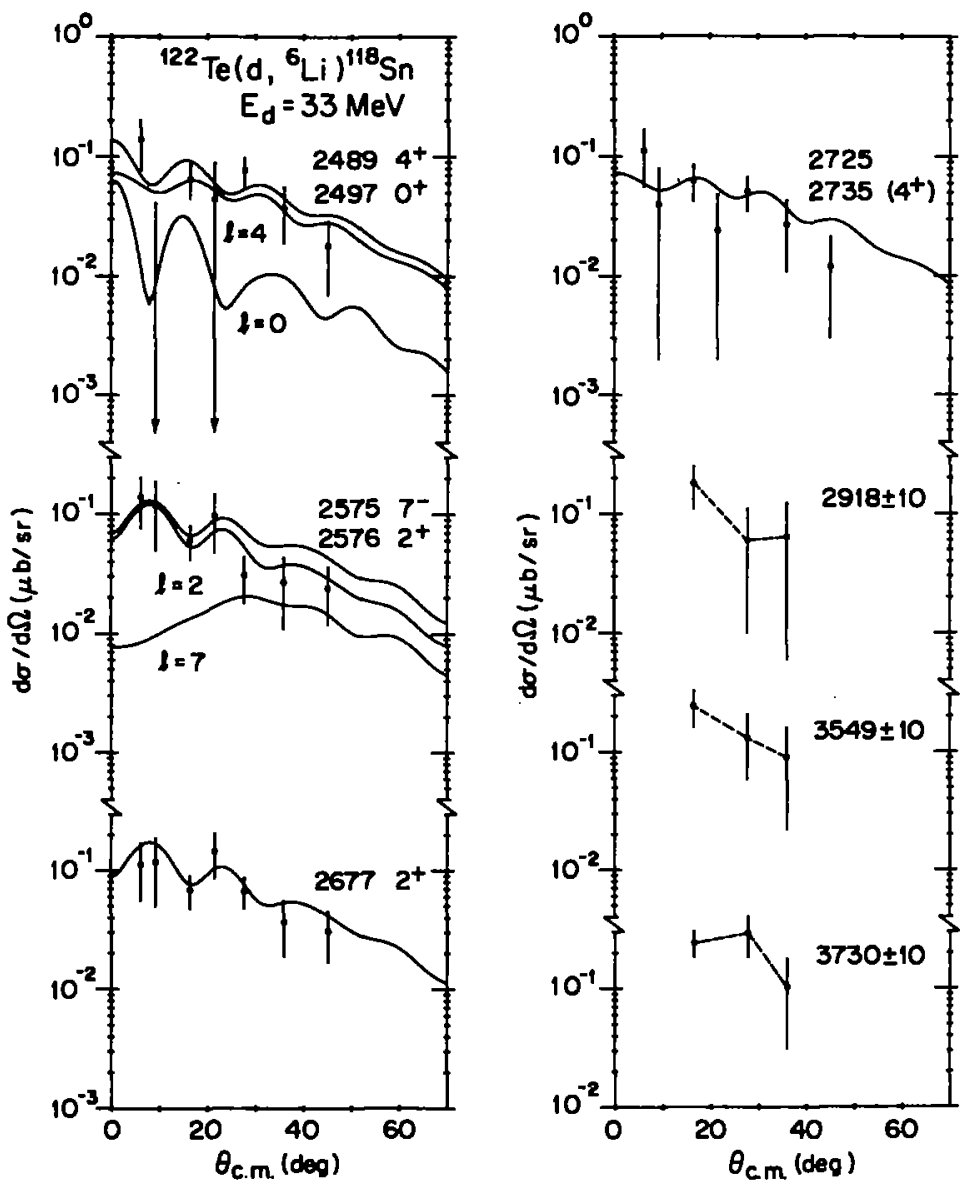

Fig. 4. See caption for fig. 3.

section ratios of the relevant states. The ratio obtained for the $3^{-} / 5^{-}$doublet in ${ }^{118} \mathrm{Sn}$, for example, agrees very well with the ratio deduced in the $(p, t)$ experiment $\left.{ }^{16}\right)$. Similar agreement was found for other doublets or multiplets. Many previously unknown states are observed, and the excitation energies are given with their experimental uncertainties. New spin-parity assignments are based on angular distributions $\left({ }^{122} \mathrm{Te}\right.$ target only) and on the systematics of the $N$-dependence of excitation energies and the absolute and relative cross sections. The spin-parity assignments of $4^{+}$and $5^{-}$for certain low excited states in ${ }^{124,126} \mathrm{Sn}$ appear to be at variance with the $l$-values from $(t, p)$ work ${ }^{27.28}$ ) but agree with assignments from inelastic proton scattering ${ }^{30}$ ).

The $\alpha$-particle apectroscopic factors $S_{\alpha}$ and reduced width $\gamma_{\alpha}^{2}$ of tables 1 to 11 will be discussed later. 
TABLE 1

Differential cross sections $\mathrm{d} \sigma / \mathrm{d} \Omega$, spectroscopic factors $S_{z}$ and reduced widths $\eta_{z}^{2}(s)$ and $\theta_{z}^{2}(s)$ for the reaction ${ }^{122} \mathrm{Te}\left(\mathrm{d},{ }^{6} \mathrm{Li}\right){ }^{118} \mathrm{Sn}$

\begin{tabular}{|c|c|c|c|c|c|c|c|c|c|}
\hline $\begin{array}{c}\left.E_{\mathbf{x}}{ }^{2}\right) \\
(\mathrm{keV})\end{array}$ & $\left.J^{\pi b}\right)$ & $J^{x}=$ & $\begin{array}{r}\mathrm{d} \sigma / \mathrm{d} \boldsymbol{\Omega}_{\mathrm{c.m}} \\
(\mu \mathrm{b} / \mathrm{sr})\end{array}$ & $\left(16^{\circ}\right)$ & $\left.S_{q^{d}}\right)$ & & $\left.N^{e}\right)$ & $\begin{array}{c}\left.\gamma_{a}^{2}(s)^{r}\right) \\
(\mathrm{eV})\end{array}$ & $\begin{array}{l}\left.\theta_{\alpha}^{2}(s)^{r}\right) \\
\left(10^{-3}\right)\end{array}$ \\
\hline 0 & $0^{+}$ & & $3.170 \pm 0.150$ & & 0.022 & $(0.022)$ & 8 & 404 & 1.74 \\
\hline 1230 & $2^{+}$ & & $0.166 \pm 0.031$ & & 0.012 & $(0.013)$ & 6 & 96 & 0.41 \\
\hline 1758 & $0^{+}$ & & $1.323 \pm 0.089$ & & 0.018 & $(0.020)$ & 7 & 154 & 0.66 \\
\hline 2043 & $\left(2^{+}\right)$ & $2^{+}$ & $0277+0030$ & $56 \%$ & 0.006 & & 6 & 43 & 0.19 \\
\hline 2057 & $0^{+}$ & & \pm 0.029 & $44 \%$ & 0.001 & & 7 & 11 & 0.05 \\
\hline 2120 & & $\left.\left(2^{+}\right)^{b}\right)$ & $0.010 \pm 0.006$ & & $\left.(0.0006)^{b}\right)$ & & 6 & (4) & $(0.02)$ \\
\hline 2280 & $4^{+}$ & & $0.087 \pm 0.023$ & & 0.008 & $(0.008)$ & 5 & 37 & 0.16 \\
\hline 2310 & $\left(3^{-}\right)$ & $3^{-}$ & & $\left.60 \%{ }^{8}\right)$ & 0.015 & $(0.017)$ & 6 & 120 & 0.52 \\
\hline 2321 & $5^{-}$ & & $0.590 \pm 0.046$ & $40 \%$ & 0.022 & $(0.026)$ & 5 & 121 & 0.52 \\
\hline 2327 & & & & & & & & & \\
\hline 2403 & $\left(2^{+}\right)$ & & $0.026+0.010$ & & $(0.002)$ & & 6 & (14) & $(0.06)$ \\
\hline 2405 & & & $0.020 \pm 0.010$ & & & & & & \\
\hline 2489 & $\left(4^{+}\right)$ & $4^{+}$ & $0.065+0.020$ & & 0.005 & & 5 & 22 & 0.10 \\
\hline 2497 & $0^{+}$ & & $0.005 \pm 0.020 \leqq$ & $\left.35 \%^{8}\right)$ & 0.0009 & & 7 & $\leq 6$ & $\leqq 0.03$ \\
\hline 2575 & $7^{-}$ & & 0. & $\left.20 \%^{8}\right)$ & 0.006 & & 4 & 18 & 0.08 \\
\hline 2576 & & $2^{+}$ & 0.0 & $\left.80 \%^{8}\right)$ & 0.003 & & 6 & 22 & 0.09 \\
\hline 2677 & $\left(2^{+}\right)$ & $2^{+}$ & $0.069 \pm 0.021$ & & 0.005 & & 6 & 31 & 0.13 \\
\hline 2725 & & & $0.063+0.0$ & & & & & & \\
\hline 2735 & $\left(4^{+}\right)$ & & $0.063 \pm 0.021$ & & $(0.006)$ & & 5 & (26) & $(0.11)$ \\
\hline $2918 \pm 15$ & & & $0.180 \pm 0.070$ & & & & & & \\
\hline $3549 \pm 15$ & & & $0.243 \pm 0.082$ & & & & & & \\
\hline $3730 \pm 15$ & & & $0.242 \pm 0.060$ & & & & & & \\
\hline
\end{tabular}

$Q=401 \mathrm{keV}\left(\right.$ All $Q$-values from ref. $\left.{ }^{41}\right)$ ).

-) Newly assigned states are given with experimental uncertainties. Known states are from the most recent Nuclear Data Sheets: ${ }^{118} \mathrm{Sn}: 17$ (1976) $1 ;{ }^{120} \mathrm{Sn}: 17$ (1976) $39 ;{ }^{122} \mathrm{Sn}: 7$ (1972) 49; ${ }^{124} \mathrm{Sn}: 10$ (1973) 91; ${ }^{126} \mathrm{Sn}: 9$ (1973) $125 ;{ }^{108} \mathrm{Cd}: 7$ (1972) 69; ${ }^{112} \mathrm{Cd}: 7$ (1972) 69; ${ }^{114} \mathrm{Cd}: 16$ (1975) $107 ;{ }^{116} \mathrm{Cd}: 14$ (1975) 247; ${ }^{118} \mathrm{Cd}: 17$ (1976) $1 ;{ }^{120} \mathrm{C}: 17$ (1976) 39.

b) Spin-parity assignment from Nuclear Data Sheets (see footnote a).

c) Spin-parity assignments from this work.

d) Spectroscopic factors from finite range calculations normalized independently to the $\alpha$-decay of ${ }^{148} \mathrm{Sm}$ are given in parenthesis.

) Assumed number of radial nodes in the $\alpha$-cluster wave function.

f) The channel radius was taken as $s=1.7 A^{1 / 3} \mathrm{fm}$.

) Relative contributions obtained from angular distributions and/or cross section systematics of corresponding states in neighboring isotopes.

h) Compatible with $J^{\pi}=2^{+}$and $S_{x} \approx 0.0006$.

\subsection{THE Sn(d, $\left.{ }^{6} \mathrm{Li}\right) \mathrm{Cd}$ REACTIONS}

Tables 6 to 11 list the differential cross sections for the Sn targets. Excitation energies of new levels and spin-parity assignments are indicated. In addition, the $Q$-value for the reaction ${ }^{124} \mathrm{Sn}\left(\mathrm{d},{ }^{6} \mathrm{Li}\right){ }^{120} \mathrm{Cd}$ and the mass excess of ${ }^{120} \mathrm{Cd}$ have been measured. The result is shown in table 12 together with two earlier measurements ${ }^{3,31}$ ). Several 
TABLE 2

${ }^{124} \mathrm{Te}\left(\mathrm{d},{ }^{6} \mathrm{Li}\right){ }^{120} \mathrm{Sn}$

\begin{tabular}{|c|c|c|c|c|c|c|c|}
\hline $\begin{array}{r}\left.E_{\mathrm{x}}{ }^{\mathrm{a}}\right) \\
(\mathrm{keV})\end{array}$ & $\left.J^{2} b\right)$ & $\left.J^{x c}\right)$ & $\begin{array}{c}\mathrm{d} \sigma / \mathrm{d} \Omega_{\text {c.m. }}\left(16^{\circ}\right) \\
(\mu \mathrm{b} / \mathrm{s} r)\end{array}$ & $S_{x}$ & $\left.N^{e}\right)$ & $\begin{array}{c}\left.\gamma_{2}^{2}(s)^{f}\right) \\
(\mathrm{eV})\end{array}$ & $\begin{array}{l}\left.\theta_{2}^{2}(s)^{f}\right) \\
\left(10^{-3}\right)\end{array}$ \\
\hline 0 & $0^{+}$ & & $2.416 \pm 0.168$ & 0.021 & 8 & 331 & 1.49 \\
\hline 1175 & $2^{+}$ & & $0.175 \pm 0.045$ & 0.011 & 6 & 71 & 0.31 \\
\hline 1875 & $0^{+}$ & & $0.373 \pm 0.066$ & 0.008 & 7 & 51 & 0.22 \\
\hline 2098 & $\left(2^{+}\right)$ & & $0.047 \pm 0.023$ & 0.004 & 6 & 21 & 0.09 \\
\hline 2160 & $0^{+}$ & & $50 \%$ & $\sim 0.002$ & 7 & $\sim 10$ & $\sim 0.05$ \\
\hline 2173 & & & $0.151 \pm 0.042$ & & & & \\
\hline 2195 & $4^{+}$ & & $50 \%$ & $\sim 0.007$ & 5 & $\sim 29$ & $\sim 0.13$ \\
\hline 2285 & $5^{-}$ & & $0.175 \pm 0.045$ & $\$ 0.023$ & 5 & $\leqslant 103$ & $\$ 0.45$ \\
\hline 2290 & $(0,1)^{+}$ & & - -0 & & & & \\
\hline $\begin{array}{l}(2323) \\
2356\end{array}$ & $2^{+}$ & & $0.128 \pm 0.039$ & $\leqq 0.011$ & 6 & $\leqq 59$ & $\leqq 0.26$ \\
\hline 2400 & $3^{-}$ & & $90 \%$ & $\sim 0.018$ & 6 & $\sim 114$ & $\sim 0.50$ \\
\hline 2421 & $(1,2)^{+}$ & & $10 \%$ & $(\sim 0.001)$ & 6 & $(\sim 4)$ & $(\sim 0.02)$ \\
\hline 2466 & $\left(4^{+}\right)$ & & $0.094+0.033 \sim 40 \%)$ & $(\sim 0.004)$ & 5 & $(\sim 15)$ & $(\sim 0.07)$ \\
\hline 2482 & $7^{-}$ & & $0.074 \pm 0.003 \sim 60 \%$ & $\sim 0.032$ & 4 & $\sim 83$ & $\sim 0.36$ \\
\hline 2548 & & & $0.047 \pm 0.023$ & & & & \\
\hline 2587 & $\left(0^{+}\right)$ & & & $(\sim 0.001)$ & 7 & $(\sim 7)$ & $(\sim 0.03)$ \\
\hline 2643 & $4^{+}$ & & $0.011 \pm 0.011$ & 0.001 & 5 & 4 & 0.02 \\
\hline 2697 & $\left(4^{+}\right)$ & & $0058+0026$ & $(\leqq 0.007)$ & 5 & $(\leqq 25)$ & $(\leqq 0.11)$ \\
\hline 2721 & $2^{+}$ & & 0.030 & $\leqq 0.006$ & 6 & $\leqq 28$ & $\leqq 0.12$ \\
\hline
\end{tabular}

$Q=-367 \mathrm{keV}$.

For table caption and footnotes ")

TABLE 3

${ }^{126} \mathrm{Te}\left(\mathrm{d},{ }^{6} \mathrm{Li}\right){ }^{122} \mathrm{Sn}$

\begin{tabular}{|c|c|c|c|c|c|c|c|}
\hline $\begin{array}{c}\left.E_{\mathrm{x}}{ }^{\prime}\right) \\
(\mathrm{keV})\end{array}$ & $\left.J^{x b}\right)$ & $\left.J^{x c}\right)$ & $\begin{array}{c}\mathrm{d} \sigma / \mathrm{d} \Omega_{\mathrm{c} \cdot \mathrm{m}}\left(16^{\circ}\right) \\
(\mu \mathrm{b} / \mathrm{sr})\end{array}$ & $S_{a}$ & $\left.N^{e}\right)$ & $\begin{array}{c}\left.\gamma_{x}^{2}(s)^{\mathrm{f}}\right) \\
(\mathrm{eV})\end{array}$ & $\begin{array}{l}\left.\theta_{g}^{2}(s)^{f}\right) \\
\left(10^{-3}\right)\end{array}$ \\
\hline 0 & $0^{+}$ & & $1.557 \pm 0.088$ & 0.015 & 8 & 202 & 0.89 \\
\hline 1140 & $2^{+}$ & & $0.194 \pm 0.031$ & 0.015 & 6 & 83 & 0.37 \\
\hline 2090 & $\left(0^{+}\right)$ & $0^{+}$ & $0.114 \pm 0.019$ & 0.003 & 7 & 17 & 0.08 \\
\hline 2145 & $\left(4^{+}\right)$ & $4^{+}$ & $0.058 \pm 0.012$ & 0.007 & 5 & 25 & 0.11 \\
\hline 2249 & $\left(5^{-}\right)$ & $5-$ & $0.105 \pm 0.015$ & 0.019 & 5 & 71 & 0.31 \\
\hline 2336 & $\left(4^{+}\right)$ & & $0.043 \pm 0.011$ & $(0.006)$ & 5 & (20) & $(0.09)$ \\
\hline 2400 & & $7^{-}$ & $0.113+0.015 \sim 50 \%$ & $\sim 0.038$ & 4 & $\sim 83$ & $\sim 0.36$ \\
\hline 2415 & $\left(2^{+}\right)$ & & $0.113 \pm 0.015 \sim 50 \%$ & $(\sim 0.006)$ & 6 & $(\sim 27)$ & $(\sim 0.12)$ \\
\hline 2492 & $3^{-}$ & & $0.311 \pm 0.026$ & 0.025 & 6 & 130 & 0.57 \\
\hline 2558 & & & $0.035 \pm 0.010$ & & & & \\
\hline $\begin{array}{l}2654 \\
2684\end{array}$ & $\left(0^{+}\right)$ & & $0.070 \pm 0.014$ & $(\leq 0.002)$ & 7 & $(\leq 11)$ & $(\leq 0.05)$ \\
\hline 2750 & & & $0.083 \pm 0.015$ & & & & \\
\hline $3319 \pm 25$ & & & $0.103 \pm 0.030$ & & & & \\
\hline $3714 \pm 25$ & & & $0.076 \pm 0.026$ & & & & \\
\hline
\end{tabular}

$Q=-1073 \mathrm{keV}$.

For table caption and footnotes ") ") table 1. 
TABLE 4

${ }^{128} \mathrm{Te}\left(\mathrm{d},{ }^{6} \mathrm{Li}\right){ }^{124} \mathrm{Sn}$

\begin{tabular}{|c|c|c|c|c|c|c|c|c|}
\hline $\begin{array}{r}\left.E_{\mathrm{x}}{ }^{\prime}\right) \\
(\mathrm{keV})\end{array}$ & $\left.J^{\pi b}\right)$ & $\left.J^{x c}\right)$ & $\begin{array}{r}\mathrm{d} \sigma / \mathrm{d} \boldsymbol{\Omega}_{\mathrm{c} . \mathrm{m}} \\
(\mu \mathrm{b} / \mathrm{sr}\end{array}$ & $\left.16^{\circ}\right)$ & $S_{\alpha}$ & $\left.N^{e}\right)$ & $\begin{array}{c}\left.\gamma_{\alpha}^{2}(s)^{\mathrm{f}}\right) \\
(\mathrm{eV})\end{array}$ & $\begin{array}{c}\left.\theta_{a}^{2}(s)^{f}\right) \\
\left(10^{-3}\right)\end{array}$ \\
\hline 0 & $0^{+}$ & & $0.987 \pm 0.049$ & & 0.011 & 8 & 127 & 0.57 \\
\hline 1131 & $2^{+}$ & & $0.156 \pm 0.020$ & & 0.015 & 6 & 68 & 0.30 \\
\hline $\begin{array}{l}2109 \\
2130\end{array}$ & & $4^{+}$ & $0.088 \pm 0.015$ & $\begin{array}{l}50 \% \\
\left.50 \%^{\prime \prime}\right)\end{array}$ & $\sim 0.007$ & 5 & $\sim 21$ & $\sim 0.09$ \\
\hline 2213 & & $5^{-}$ & $0.103 \pm 0.016$ & & 0.024 & 5 & 78 & 0.35 \\
\hline $\left.2300^{h}\right)$ & & $\left(0^{+}\right)$ & $<0.027$ & & $<0.001$ & 7 & $<5$ & $<0.02$ \\
\hline 2333 & $\left(7^{-}\right)$ & $7^{-}$ & $0.102 \pm 0.016$ & & 0.087 & 4 & 161 & 0.72 \\
\hline $\begin{array}{l}2438 \\
2455\end{array}$ & $\left(2^{+}\right)$ & & $0.061 \pm 0.013$ & & $(\leqq 0.008)$ & 6 & $(\leqq 29)$ & $(\leqq 0.13)$ \\
\hline 2612 & $3^{-}$ & & $0.227 \pm 0.024$ & & 0.023 & 6 & 102 & 0.46 \\
\hline $\begin{array}{l}2690 \\
2713\end{array}$ & & & $0.039 \pm 0.010$ & & & & & \\
\hline 2900 & & & $0.048 \pm 0.011$ & & & & & \\
\hline
\end{tabular}

$Q=-1703 \mathrm{keV}$.

For table caption and footnotes ${ }^{\text {a) }}{ }^{8}$ ) see table 1.

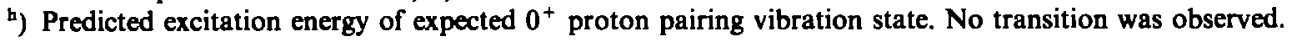
The upper limit for the spectroscopic strength is $S_{\alpha}<0.001$.

TABLE 5

${ }^{130} \mathrm{Te}\left(\mathrm{d},{ }^{6} \mathrm{Li}\right){ }^{126} \mathrm{Sn}$

\begin{tabular}{|c|c|c|c|c|c|c|c|}
\hline $\begin{array}{c}\left.E_{\mathrm{x}}^{4}\right) \\
(\mathrm{keV})\end{array}$ & $J^{\pi b}$ ) & $\left.J^{* c}\right)$ & $\begin{array}{c}\mathrm{d} \sigma / \mathrm{d} \Omega_{\mathrm{c}, \mathrm{m}}\left(16^{\circ}\right) \\
(\mu \mathrm{b} / \mathrm{sr})\end{array}$ & $S_{z}$ & $\left.N^{*}\right)$ & $\begin{array}{c}\left.\gamma_{a}^{2}(s)^{f}\right) \\
(\mathrm{eV})\end{array}$ & $\begin{array}{l}\left.\theta_{a}^{2}(s)^{f}\right) \\
\left(10^{-3}\right)\end{array}$ \\
\hline 0 & $0^{+}$) & & $0.406 \pm 0.049$ & 0.006 & 8 & 53 & 0.24 \\
\hline 1145 & $\left(2^{+}\right)$ & $2^{+}$ & $0.135 \pm 0.028$ & 0.015 & 6 & 60 & 0.27 \\
\hline 2054 & $\left(5^{-}\right)$ & $\left(4^{+}\right)$ & $0.032 \pm 0.009$ & $(0.007)$ & 5 & (17) & $(0.08)$ \\
\hline 2167 & $\left(6^{+}\right)$ & $5^{-}$ & $0.074 \pm 0.013$ & 0.022 & 5 & 62 & 0.28 \\
\hline 2222 & $\left(7^{-}\right)$ & $7^{-}$ & $0.079 \pm 0.013$ & 0.076 & 4 & 123 & 0.55 \\
\hline $2298 \pm 25$ & & & $0.016 \pm 0.006$ & & & & \\
\hline 2378 & $\left(2^{+}\right)$ & & $0.039 \pm 0.010$ & $(0.006)$ & 6 & (18) & $(0.08)$ \\
\hline $2550 \pm 25$ & & & $0.023 \pm 0.007$ & & & & \\
\hline 2659 & & & $0.016 \pm 0.006$ & & & & \\
\hline 2720 & $\left(3^{-}\right)$ & $3^{-}$ & $0.053 \pm 0.012$ & 0.007 & 6 & 26 & 0.12 \\
\hline $2795 \pm 25$ & & & $0.027 \pm 0.010$ & & & & \\
\hline 2892 & & & $0.034 \pm 0.011$ & & & & \\
\hline $2971 \pm 25$ & & & $0.027 \pm 0.010$ & & & & \\
\hline 3278 & & & $0.015 \pm 0.008$ & & & & \\
\hline $3385 \pm 25$ & & & $0.030 \pm 0.011$ & & & & \\
\hline 3424 & $\left(4^{+}\right)$ & & $0.080 \pm 0.017$ & $(0.031)$ & 5 & (62) & $(0.28)$ \\
\hline 3790 & & & $0.050 \pm 0.014$ & & & & \\
\hline $3985 \pm 25$ & & & $0.046 \pm 0.013$ & & & & \\
\hline
\end{tabular}

$Q=-2276 \mathrm{keV}$.

For table caption and footnotes ")-") see table 1 . 
TABLE 6

${ }^{112} \mathrm{Sn}\left(\mathrm{d},{ }^{6} \mathrm{Li}\right){ }^{108} \mathrm{Cd}$

\begin{tabular}{|c|c|c|c|c|c|c|c|}
\hline $\begin{array}{c}\left.E_{\mathbf{1}}^{*}\right) \\
(\mathrm{keV})\end{array}$ & $J^{x}$ b) & $\left.J^{\mathbf{x}} \mathbf{c}\right)$ & $\begin{array}{c}\mathrm{d} \sigma / \mathrm{d} \Omega_{\text {c.m.m }}\left(16^{\circ}\right) \\
(\mu \mathrm{b} / \mathrm{sr})\end{array}$ & $S_{\alpha}$ & $\left.N^{e}\right)$ & $\begin{array}{c}\left.\gamma_{x}^{2}(s)^{f}\right) \\
(\mathrm{eV})\end{array}$ & $\begin{array}{l}\left.\theta_{a}^{2}(s)^{\prime}\right) \\
\left(10^{-3}\right)\end{array}$ \\
\hline 0 & $0^{+}$ & & $1.629 \pm 0.151$ & 0.019 & 7 & 279 & 1.13 \\
\hline 633 & $2^{+}$ & & $0.334 \pm 0.068$ & 0.010 & 6 & 121 & 0.49 \\
\hline 1509 & $\left(4^{+}\right)$ & $4^{+}$ & $0.181 \pm 0.050$ & 0.009 & 5 & 65 & 0.26 \\
\hline 1607 & $2^{+}$ & & $0.111+0.039$ & 0.005 & 6 & 48 & 0.19 \\
\hline $1704 \pm 25$ & & & $0.042 \pm 0.024$ & & & & \\
\hline $1830 \pm 30$ & & & $0.014 \pm 0.014$ & & & & \\
\hline $\left.1938 \pm 25^{h}\right)$ & & $\left(0^{+}\right)$ & $0.056 \pm 0.028$ & $(0.001)$ & 7 & (9) & $(0.04)$ \\
\hline $\begin{array}{l}2228 \\
2239\end{array}$ & $\left(3^{-}\right)$ & $3^{-}$ & $0.334 \pm 0.068$ & 0.011 & 6 & 123 & 0.50 \\
\hline 2414 & & & $0.056 \pm 0.028$ & & & & \\
\hline $\begin{array}{l}2541 \\
2566\end{array}$ & $(6)^{+}$ & & $0.153 \pm 0.046$ & $(0.042)$ & 4 & (166) & $(0.67)$ \\
\hline $\begin{array}{l}2602 \\
2738 \pm 25 \\
2808 \\
2921 \pm 25\end{array}$ & (5) & & $\begin{array}{l}0.362 \pm 0.071 \\
0.251 \pm 0.059 \\
0.125 \pm 0.042 \\
0.139 \pm 0.044\end{array}$ & $(0.033)$ & 5 & (237) & (0.96) \\
\hline
\end{tabular}

$Q=-357 \mathrm{keV}$.

For table caption and footnotes ")

b) Most likely candidate for the $0^{+}$proton-pairing-vibration state observed at $E_{\mathrm{x}} \approx 1.9 \mathrm{MeV}$ with the $\left({ }^{3} \mathrm{He}, \mathrm{n}\right)$ reaction $\left.{ }^{17}\right)$. The spectroscopic strength is $S_{x} \approx 0.001$.

recent mass predictions ${ }^{32}$ ) are in reasonable agreement with the experimental mass value as is expected for nuclei which are close in nucleon number to known nuclei.

\section{Systematics of excitation energies}

The energy spectra of the various $\mathrm{Sn}$ and $\mathrm{Cd}$ isotopes display a systematic dependence on neutron number $N$. Both excitation energies and cross sections for states with the same spin and parity $J^{\pi}$ vary in a rather smooth and often correlated manner. This fact was occasionally used (tables 1 to 11) to support new spin-parity assignments or to estimate relative contributions to the cross section from unresolved levels.

Fig. 5 displays theoretical and experimental excitation energies for ground and excited $0^{+}$states (upper part) and the energetically lowest or yrast states with $J^{\pi} \neq 0^{+}$ (lower part) as a function of neutron number $N$. The theoretical curves of fig. 5a are from the BCS calculations of Clement and Baranger ${ }^{18}$ ) (open circles) and from the interacting boson model of Arima and Iachello ${ }^{21-24}$ ) (filled circles). Figs. 5b and $5 \mathrm{c}$ show the experimental excitation energies for the even- $A \mathrm{Sn}$ and $\mathrm{Cd}$ isotopes. The energies are taken from recent compilations, from the present investigation and, for excited $0^{+}$states, from $\left({ }^{3} \mathrm{He}, \mathrm{n}\right)$ two-proton transfer data $\left.{ }^{17}\right)$ (filled circles).

The $0^{+}$ground states in the $\mathrm{Sn}$ isotopes can be described ${ }^{18}$ ) by zero-quasiparticle 
TABLE 7

${ }^{116} \mathrm{Sn}\left(\mathrm{d},{ }^{6} \mathrm{Li}\right){ }^{112} \mathrm{Cd}$

\begin{tabular}{|c|c|c|c|c|c|c|c|c|}
\hline $\begin{array}{c}\left.E_{\mathrm{x}}{ }^{2}\right) \\
(\mathrm{keV})\end{array}$ & $J^{x \text { b) }}$ & $J^{x}$ c) & $\begin{array}{r}\mathrm{d} \sigma / \mathrm{d} \Omega_{\text {c.m.m. }} \\
(\mu \mathrm{b} / \mathrm{s} r\end{array}$ & 16) & $S_{x}$ & $\left.N^{\circ}\right)$ & $\begin{array}{c}\left.\gamma_{x}^{2}(s)^{\mathrm{f}}\right) \\
(\mathrm{eV})\end{array}$ & $\begin{array}{l}\left.\theta_{x}^{2}(s)^{\prime}\right) \\
\left(10^{-3}\right)\end{array}$ \\
\hline 0 & $0^{+}$ & & $1.586 \pm 0.165$ & & 0.021 & 7 & 215 & 0.89 \\
\hline 617 & $2^{+}$ & & $0.431 \pm 0.086$ & & 0.023 & 6 & 184 & 0.76 \\
\hline 1223 & $0^{+}$ & & $0.103 \pm 0.042$ & & 0.002 & 7 & 14 & 0.06 \\
\hline 1312 & $2^{+}$ & & $0.069 \pm 0.034$ & & 0.005 & 6 & 34 & 0.14 \\
\hline 1414 & $4^{+}$ & & $0086+0052$ & $\left.55 \%{ }^{8}\right)$ & $\sim 0.004$ & 5 & $\sim 21$ & $\sim 0.09$ \\
\hline 1432 & $(0)^{+}$ & & $0.086 \pm 0.052$ & $45 \%$ & $(\sim 0.001)$ & 7 & $(\sim 6)$ & $(\sim 0.02)$ \\
\hline 1468 & $2^{+}$ & & $0.052 \pm 0.046$ & & 0.004 & 6 & 26 & 0.11 \\
\hline $1812 ?$ & & & $0.052 \pm 0.030$ & & & & & \\
\hline 1870 & $0^{+}$ & & $0.052 \pm 0.030$ & & 0.001 & 7 & 7 & 0.03 \\
\hline $\begin{array}{l}1971 \\
2004\end{array}$ & $3^{-}$ & & $0.345 \pm 0.077$ & & $\leqq 0.021$ & 6 & $\leqq 157$ & $\leqq 0.66$ \\
\hline $2047 ?$ & & & & & & & & \\
\hline 2063 & & & $0.121 \pm 0.046$ & & & & & \\
\hline 2087 & & & & & & & & \\
\hline $\begin{array}{l}2123 \\
2149\end{array}$ & & & $0.103 \pm 0.042$ & & & & & \\
\hline $\begin{array}{l}2149 \\
2229\end{array}$ & & & 0.017 & & & & & \\
\hline 2302 & $\left(0^{+}\right)$ & & $0.052 \pm 0.030$ & & & & & \\
\hline 2374 & & & $0.086 \pm 0.039$ & & & & & \\
\hline $\begin{array}{l}2377 \\
2415\end{array}$ & & & $0.103 \pm 0.042$ & & & & & \\
\hline 2507 & & & $0.172 \pm 0.055$ & & & & & \\
\hline 2573 & & & $0.086 \pm 0.039$ & & & & & \\
\hline 2608 & & & & & & & & \\
\hline 2637 & & & $0.172 \pm 0.055$ & & & & & \\
\hline 2657 & & & & & & & & \\
\hline
\end{tabular}

$Q=-1898 \mathrm{keV}$.

For table caption and footnotes $\left.{ }^{2}\right)^{8}$ ) see table 1 .

BCS wave functions as demonstrated by $(p, t)$ and $(t, p)$ two-neutron transfer data [refs. $\left.{ }^{16,26-29}\right)$ ]. Excited states are described ${ }^{18}$ ) by two-quasiparticle BCS wave functions based on 12 neutron orbitals and 12 proton orbitals thus allowing for core excitations. Proton excitations are restricted to particle-hole excitations. The latter contribute about $15 \%$ in intensity to the lowest $2^{+}$and about $35 \%$ to the lowest $3^{-}$states. The calculated energies of the $2^{+}$and $3^{-}$states with their weak dependence on neutron number correspond quite well to the respective experimental states in the $\mathrm{Sn}$ and also $\mathrm{Cd}$ isotopes. The relatively sharp decrease of the experimental $7^{-}$ state excitation energies in $\mathrm{Sn}$ is worth noting. It also appears that the neutron-rich isotopes ${ }^{118} \mathrm{Cd}$ and ${ }^{120} \mathrm{Cd}$ have very low excited $7^{-}$states at $E_{\mathrm{x}} \approx 1300 \mathrm{keV}$.

There is no obvious correspondence between the calculated and observed excited $0^{+}$states. From the five neutron valence orbits $0 \mathrm{~g}_{\frac{\gamma}{\gamma}}, 1 \mathrm{~d}_{\frac{1}{\xi}}, 2 \mathrm{~s}_{\frac{1}{2}}, 1 \mathrm{~d}_{\frac{3}{4}}$ and $0 \mathrm{~h}_{\frac{11}{2}}$ one expects five $0^{+}$states. One of these is the "coherent" BCS ground state and, separated by 
TABLE 8

${ }^{118} \mathrm{Sn}\left(\mathrm{d},{ }^{6} \mathrm{Li}\right){ }^{114} \mathrm{Cd}$

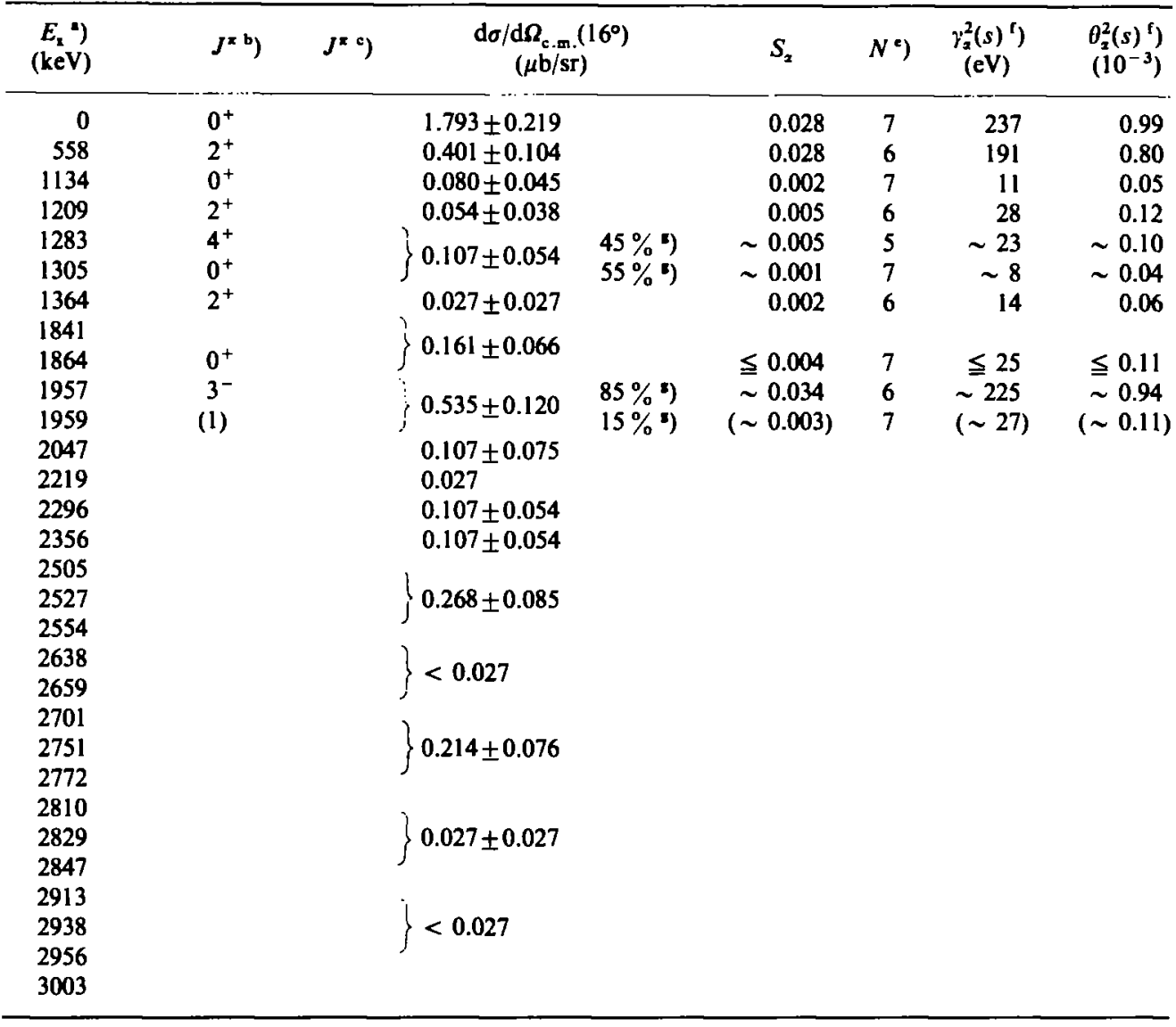

$Q=-2586 \mathrm{keV}$.

For table caption and footnotes ")-") see table 1 .

about twice the pairing gap, four more $0^{+}$states (three are shown in fig. 5a with open circles) are expected at higher excitation energy with a strong dependence of excitation on neutron number. Because of the low spin and the nature of the pairing interaction, the $0^{+}$two-quasiparticle state based on $\left(2 \mathrm{~s}_{\frac{1}{2}}\right)^{2}$ shold be relatively pure. Indeed, the states with the minimum in energy at $N \approx 66$ have about $85 \%$ of calculated $\left(2 s_{1}\right)^{2}$ strength. Similarly, the states with the minimum at lower $N$ have about $80 \%$ of combined $\left(1 \mathrm{~d}_{\frac{3}{3}}\right)^{2}$ and $\left(\lg _{\frac{7}{2}}\right)^{2}$ strength, while those with the minimum at higher $N$ have about $70 \%$ of $\left(1 \mathrm{~d}_{\frac{3}{3}}\right)^{2}$ and about $15 \%$ of $\left(\mathrm{h}_{\frac{12}{2}}\right)^{2}$ strength. Strong mixing occurs at the intersections of the three lines. The experimental $0^{+}$states in ${ }^{114,116,118,120} \mathrm{Sn}$ at 2.0 to $2.2 \mathrm{MeV}$ and in ${ }^{116,118} \mathrm{Cd}$ at 1.3 to $1.4 \mathrm{MeV}$ (open circles) may correspond 
TABLE 9

${ }^{120} \mathrm{Sn}\left(\mathrm{d},{ }^{6} \mathrm{Li}\right){ }^{116} \mathrm{Cd}$

\begin{tabular}{|c|c|c|c|c|c|c|c|c|}
\hline $\begin{array}{c}\left.E_{\mathrm{x}}{ }^{\prime}\right) \\
(\mathrm{keV})\end{array}$ & $\left.J^{x b}\right)$ & $\left.J^{\pi c}\right)$ & $\begin{array}{r}\mathrm{d} \sigma / \mathrm{d} \Omega_{\text {e.m.m. }} . \\
(\mu \mathrm{b} / \mathrm{sr}\end{array}$ & 16') & $S_{\alpha}$ & $\left.N^{*}\right)$ & $\begin{array}{c}\left.\gamma_{z}^{2}(s)^{f}\right) \\
(\mathrm{eV})\end{array}$ & $\begin{array}{l}\left.\theta_{a}^{2}(s)^{f}\right) \\
\left(10^{-3}\right)\end{array}$ \\
\hline $\mathbf{0}$ & $\mathbf{0}^{+}$ & & $1.130 \pm 0.135$ & & 0.022 & 7 & 152 & 0.64 \\
\hline 514 & $2^{+}$ & & $0.355 \pm 0.076$ & & 0.032 & 6 & 178 & 0.76 \\
\hline 1214 & $2^{+}$ & & 00 & $40 \%$ & $\sim 0.004$ & 6 & $\sim 17$ & $\sim 0.08$ \\
\hline 1220 & $4^{+}$ & & & $60 \%$ & $\sim 0.007$ & 5 & $\sim 26$ & $\sim 0.11$ \\
\hline $\left.1283^{b}\right)$ & & $\left(0^{+}\right)$ & $0.065 \pm 0.032$ & & $(0.002)$ & 7 & (11) & $(0.05)$ \\
\hline 1381 & $0^{+}$ & & $0.081 \pm 0.039$ & & 0.002 & 7 & 14 & 0.06 \\
\hline $\begin{array}{l}1641 \\
\left.(1780 \pm 25)^{\prime}\right) \\
\left.(1853 \pm 25)^{1}\right)\end{array}$ & & $\left(2^{+}\right)$ & $\begin{array}{l}0.032 \pm 0.023 \\
<0.032 \\
<0.032\end{array}$ & & $(0.004)$ & 6 & (18) & $(0.08)$ \\
\hline $\left.1920^{J}\right)$ & $\left(3^{-}\right)$ & $3^{-}$ & $0.258 \pm 0.065$ & & 0.026 & 6 & 142 & 0.60 \\
\hline 2028 & $\left(1^{-}\right)$ & & $0.032 \pm 0.023$ & & $(0.002)$ & 7 & (15) & $(0.06)$ \\
\hline 2115 & & & $<0.016$ & & & & & \\
\hline 2250 & & & $0.048 \pm 0.028$ & & & & & \\
\hline 2296 & $\left(3^{-}\right)$ & & $0.113 \pm 0.043$ & & $(0.013)$ & 6 & (68) & $(0.29)$ \\
\hline \multicolumn{9}{|l|}{2338} \\
\hline 2371 & & & \multirow{3}{*}{\multicolumn{6}{|c|}{$0.129 \pm 0.046$}} \\
\hline 2386 & & & & & & & & \\
\hline 2434 & & & & & & & & \\
\hline
\end{tabular}

$Q=-3334 \mathrm{keV}$.

For table caption and footnotes ")-") see table 1.

b) Likely candidate for the $0^{+}$proton pairing vibration state.

') States uncertain.

J) $\gamma$-deexcitation from states at 1917,1923 and $1930 \mathrm{keV}$ has been measured recently ${ }^{83}$ ) and only the decay of the state at $1923 \mathrm{keV}$ was found to be consistent with a $3^{-}$assignment.

TABLE 10

${ }^{122} \mathrm{Sn}\left(\mathrm{d},{ }^{6} \mathrm{Li}\right){ }^{118} \mathrm{Cd}$

\begin{tabular}{|c|c|c|c|c|c|c|c|}
\hline $\begin{array}{r}\left.E_{\mathrm{z}}^{2}\right) \\
(\mathrm{keV})\end{array}$ & $J^{x}$ b) & $\left.J^{\pi c}\right)$ & $\underset{(\mu \mathrm{b} / \mathrm{sr})}{\mathrm{d} \sigma / \mathrm{d} \Omega_{\mathrm{c.m}}}\left(16^{\circ}\right)$ & $S_{2}$ & $\left.N^{\top}\right)$ & $\begin{array}{c}\left.\gamma_{a}^{2}(s) f\right) \\
(e V)\end{array}$ & $\begin{array}{l}\left.\theta_{a}^{2}(s)^{f}\right) \\
\left(10^{-3}\right)\end{array}$ \\
\hline 0 & $0^{+}$ & & $0.724 \pm 0.077$ & 0.019 & 7 & 109 & 0.46 \\
\hline 488 & $2^{+}$ & & $0.324 \pm 0.051$ & 0.037 & 6 & 170 & 0.73 \\
\hline 1165 & & & $0.050 \pm 0.022$ & & & & \\
\hline $\begin{array}{l}1269 \\
1286\end{array}$ & & $\left(7^{-}\right)$ & $0.271 \pm 0.047$ & & & & \\
\hline $\begin{array}{l}\left.1460 \pm 30^{b}\right) \\
1600 \pm 30\end{array}$ & & $\left(0^{+}\right)$ & $\begin{array}{l}0.050 \pm 0.022 \\
0.053 \pm 0.022\end{array}$ & $(0.002)$ & 7 & $(10)$ & $(0.04)$ \\
\hline $\begin{array}{l}1935 \\
1973\end{array}$ & & $3^{-}$ & $0.456 \pm 0.064 \quad 90 \%$ & $\sim 0.061$ & 6 & $(\sim 263)$ & $(\sim 1.13)$ \\
\hline $\begin{array}{l}2110 \pm 30 \\
2223 \\
2395 \pm 30 \\
2575 \pm 30\end{array}$ & & & $\begin{array}{r}0.090 \pm 0.032 \\
0.034 \pm 0.020 \\
0.158 \pm 0.042 \\
0.101 \pm 0.034\end{array}$ & & & & \\
\hline
\end{tabular}

$Q=-4189 \mathrm{keV}$.

For table caption and footnotes ") see table 1.

h) Candidate for the $\mathrm{O}^{+}$proton pairing vibration state. 
TABLE 11

${ }^{124} \mathrm{Sn}\left(\mathrm{d},{ }^{6} \mathrm{Li}\right){ }^{120} \mathrm{Cd}$

\begin{tabular}{|c|c|c|c|c|c|c|c|}
\hline $\begin{array}{c}\left.E_{\mathrm{x}}{ }^{\mathrm{q}}\right) \\
(\mathrm{keV})\end{array}$ & $\left.J^{* \mathbf{b}}\right)$ & $\left.J^{* c}\right)$ & $\underset{(\mu \mathrm{b} / \mathrm{sr})}{\mathrm{d} \sigma / \mathrm{d} \Omega_{\text {c.m. }}\left(16^{\circ}\right)}$ & $S_{\alpha}$ & $\left.N^{\circ}\right)$ & $\begin{array}{c}\left.\gamma_{x}^{2}(s)^{\mathrm{f}}\right) \\
(\mathrm{keV})\end{array}$ & $\begin{array}{r}\left.\theta_{a}^{2}(s)^{\mathrm{f}}\right) \\
\left(10^{-3}\right)\end{array}$ \\
\hline 0 & $0^{+}$ & & $0.475 \pm 0.052$ & 0.017 & 7 & 83 & 0.36 \\
\hline 506 & $2^{+}$ & & $0.237 \pm 0.037$ & 0.032 & 6 & 123 & 0.53 \\
\hline$\left.(975 \pm 25)^{b}\right)$ & & & $0.062 \pm 0.023$ & & & & \\
\hline 1204 & & & $0.041 \pm 0.017$ & & & & \\
\hline 1323 & & $\left(7^{-}\right)$ & $0.315 \pm 0.042$ & & & & \\
\hline $1920 \pm 25$ & & $3^{-}$ & $0.291 \pm 0.051$ & 0.055 & 6 & 205 & 0.89 \\
\hline 2034 & & & $0.088 \pm 0.028$ & & & & \\
\hline
\end{tabular}

$Q=-5202 \pm \mathrm{keV} ; Q$-value from this work and refs. ${ }^{3,31,41}$ ).

For table caption and footnotes ")-") see table 1.

b) State uncertain.

to the calculated two-quasiparticle states, the latter with two proton holes coupled to it.

Two classes of excited states are not included in the BCS calculations as they are not part of the assumed basis. The lowest $2^{+}$states calculated at $E_{\mathrm{x}} \approx 1.0 \mathrm{MeV}$ are "coherent" two-quasiparticle states (seniority $v=2$ ). However, higher excited states with $v>2$ and similar characteristics do also exist. In the vibrational limit these "coherent" states are called two- and three-phonon quadrupole vibrational states, and their excitation energies are then integer multiples of those of the onephonon $2^{+}$state at $E_{\mathrm{x}} \gtrless 1.0 \mathrm{MeV}$ in $\mathrm{Sn}$ and $E_{\mathrm{x}} \gtrless 0.5 \mathrm{MeV}$ in Cd. The $0^{+}$states mentioned above (open circles) in certain $\mathrm{Sn}$ and $\mathrm{Cd}$ isotopes are also candidates for this type of excitation. Similar comments apply to the lowest $3^{-}$octupole vibration states calculated at $E_{\mathrm{x}} \approx 2.1 \mathrm{MeV}$. All these states have in common the fact that their excitation energies change only weakly with neutron number ${ }^{33}$ ).

Another important class of excited states is that which includes proton pair excitations. Of these states, the $0^{+}$proton pairing vibration state is the most interesting one. It is expected ${ }^{13,20}$ ) to carry about $85 \%$ of $\left(0 \mathrm{~g}_{\frac{7}{2}}\right)^{2}$ and $\left(1 \mathrm{~d}_{\frac{1}{2}}\right)^{2}$ strength (proton addition phonons) and about $80 \%$ of $\left(\mathrm{Og}_{\mathrm{g}}\right)^{-2}$ strength (proton removal phonons) coupled to the neutron BCS g.s. Much of the expected proton pairing strength has been observed in the $\mathrm{Cd}\left({ }^{3} \mathrm{He}, \mathrm{n}\right)$ reaction $\left.{ }^{17}\right)$ with $\gtrsim 50 \%$ of the fragmented strength concentrated in the energetically lowest $0^{+}$component. The low excitation energy and the quadratic dependence on neutron number with a minimum near the middle of the neutron shell is not fully understood. However, it appears that the interacting boson approximation (IBA) of Arima and Iachello $21-24.34-37$ ) provides a semiquantitative phenomenological description of the observed excitation energies and transition strengths.

The excitation energies $E_{x}$ of neutron pairing vibration states are given by the 
TABLE 12

$Q$-values for ${ }^{124} \mathrm{Sn}\left(\mathrm{d},{ }^{6} \mathrm{Li}\right){ }^{120} \mathrm{Cd}$ and mass excess for ${ }^{120} \mathrm{Cd}$

\begin{tabular}{|c|c|c|c|}
\hline$\underset{(\mathrm{keV})}{Q}$ & $\begin{array}{l}\Delta M \\
(\mathrm{keV})\end{array}$ & $\begin{array}{c}\Delta M(\mathrm{calc})-\Delta M(\exp ) \\
(\mathrm{keV})\end{array}$ & Ref. \\
\hline \multicolumn{4}{|l|}{ experimental: } \\
\hline $\begin{array}{l}-5210 \pm 30 \\
-5186 \pm 22 \\
-5216 \pm 24 \\
-5202 \pm 14\end{array}$ & $\begin{array}{l}-83981 \pm 30 \\
-84004 \pm 23 \\
-83975 \pm 25 \\
-83988 \pm 15\end{array}$ & & $\begin{array}{l}31,41 \text { ) } \\
{ }^{3} \text { ) } \\
\text { this work } \\
\text { average }\end{array}$ \\
\hline calculated: & $\begin{array}{l}-85140 \\
-85010 \\
-84000 \\
-83890 \\
-81900 \\
-84050 \\
-84370 \pm 840 \\
-84310\end{array}$ & $\begin{array}{r}-1152 \\
-1022 \\
-12 \\
98 \\
2088 \\
-62 \\
-382 \\
-322\end{array}$ & $\begin{array}{l}\left.3^{32}\right)(\mathrm{M}) \\
\left.{ }^{32}\right)(\mathrm{GHT}) \\
\left.{ }^{32}\right)(\mathrm{SH}) \\
\left.{ }^{32}\right)(\mathrm{LZ}) \\
\left.{ }^{32}\right)(\mathrm{BLM}) \\
3^{32} \text { ) (JGK) } \\
\left.{ }^{32}\right)(\mathrm{CK}) \\
32)(\mathrm{JE})\end{array}$ \\
\hline
\end{tabular}

well-known expression ${ }^{38}$ )

$$
E_{\mathbf{z}}^{\mathrm{vib}}(Z, N)=E(Z, N+2)-2 E(Z, N)+E(Z, N-2),
$$

while for proton pairing vibration states the energy is reduced due to the Coulomb contributions to the particle-hole interaction ${ }^{39.40}$ ),

$$
E_{\mathrm{x}}^{\mathrm{vib}}(Z, N)=E(Z+2, N)-2 E(Z, N)+E(Z-2, N)-4 E(\mathrm{p}-\mathrm{h}) .
$$

Here, $E(Z, N)$ is the ground state and $E(\mathrm{p}-\mathrm{h})$ the particle-hole energy. Flynn and $\mathrm{Kunz}^{40}$ ) have estimated the excitation energies for the proton pairing vibration states in the $\mathrm{Sn}$ isotopes as $E_{\mathrm{x}}^{\mathrm{vib}} \approx 3.1 \mathrm{MeV}$. Yet it is only for ${ }^{100} \mathrm{Sn}$ and ${ }^{132} \mathrm{Sn}$ that these states should actually appear at the calculated energy with $100 \%$ of the expected pairing strength. When neutron pairs are added the strong proton-neutron interaction will lead to increased collectivity (deformation), particularly since proton and neutron pairs occupy the same shell-model orbits. This will reduce the pairing strength and lower the excitation energies according to eq. (2.30) of ref. ${ }^{22}$ ) or eq. (9) of ref. ${ }^{35}$ )

$$
E_{\mathbf{x}}(Z, N)=E_{\mathbf{x}}^{\mathrm{vib}}(Z, N)-\kappa\left(4 n^{2}+6 n\right) \text {. }
$$

Here, $\kappa$ is the strength of the boson quadrupole-quadrupole interaction ${ }^{22,35}$ ) and $n=n_{\mathrm{k}}+n_{\mathrm{v}}$ is the number of "active" proton and neutron bosons (or boson holes beyond the middle of the neutron shell). Eq. (3) describes the experimental excitations energies quite well (see filled circles in fig. 5a and thin line in fig. 5b). The data do not 


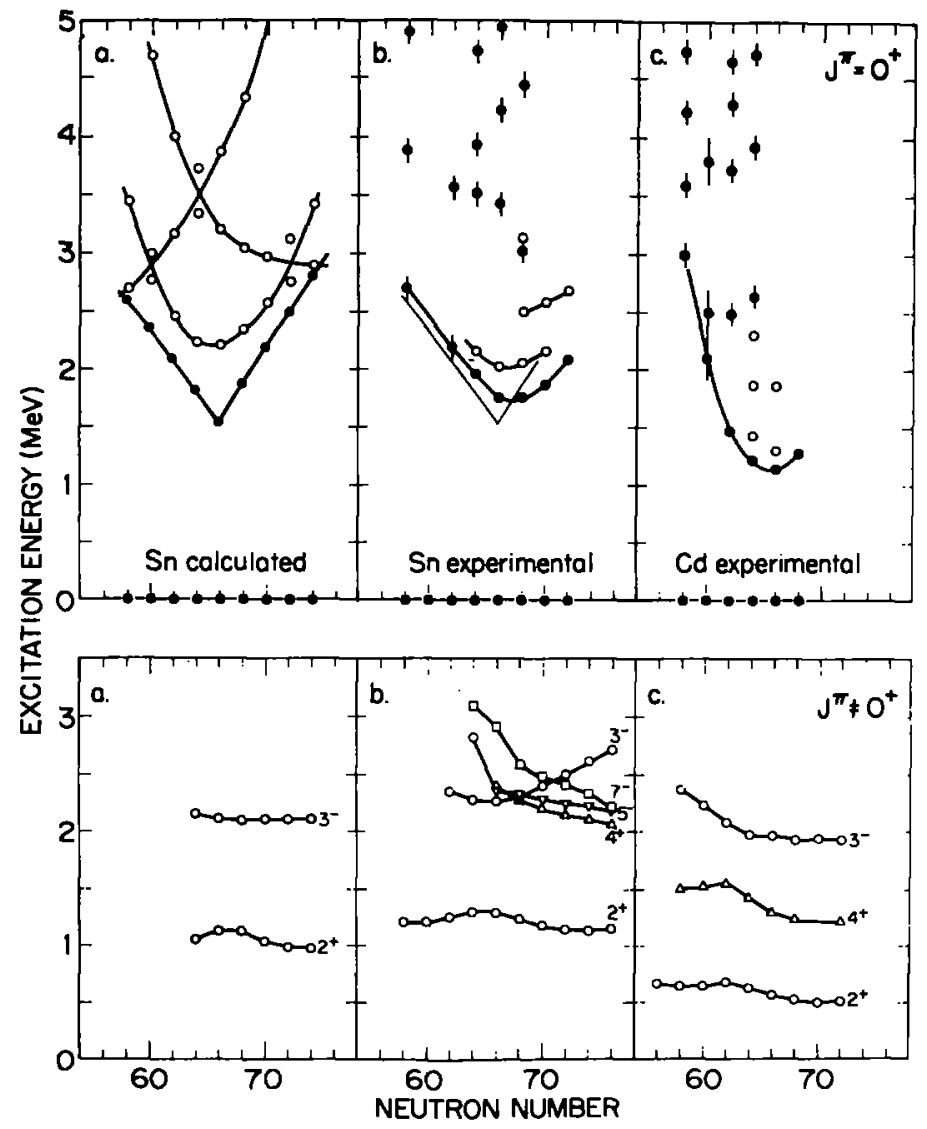

Fig. 5. Calculated and experimental excitation energies for states in $\mathrm{Sn}$ and $\mathrm{Cd}$ isotopes. TOP: $0^{+}$states; BOTTOM : energetically lowest or yrast states with $J^{x}>0$. (a) Sn isotopes, calculated (open circles BCS; filled circles IBA; see text); (b) Sn isotopes, experimental; (c) Cd isotopes, experimental. The experimental $0^{+}$states given as filled circles are seen in two-proton stripping ${ }^{17}$ ). The lines connecting data points are included to guide the eye.

show the downward spike in the middle of the neutron shell. This is not too serious as here the proton boson-neutron boson coupling changes its character from particleparticle to particle-hole. Also, the interaction strength $\kappa$ may depend on boson number. The vibrational contributions to eq. (3) were calculated with eq. (2) using experimental masses of ground states ${ }^{41}$ ), predictions from the Garvey-Kelson mass equation ${ }^{32}$ ) as well as the value for the particle-hole interaction of Flynn and $\mathrm{Kunz}^{40}$ ). For simplicity the values so obtained were fitted by a quadratic expression $\left(E_{\mathbf{x}}^{\mathbf{v}}=\right.$ $3.55,3.05,3.95 \mathrm{MeV}$ for $N=50,66,82$, respectively). The rotational contribution was calculated with $\kappa=\mathbf{4 k e V}$. This value is smaller than for the extreme rotational limit of the interacting boson approximation ${ }^{23}$ ). The situation is reminiscent of the 
$\mathrm{Sm}$ isotopes ${ }^{24}$ ) with $\kappa=9.75 \mathrm{keV}$ and the associated transition from vibrational to rotational nuclei. The static deformation for these $0^{+}$states has been observed experimentally ${ }^{42}$ ). The states constitute the band heads of rotational bands, and the deformation reaches a maximum near the middle of the neutron shell where the band head excitation energy is lowest. However, the level spacing indicates softness against deformation ${ }^{42}$ ) which is characteristic for transitional nuclei rather than for closed shell nuclei. Whereas rotational bands based on the corresponding $0^{+}$ states in the neighboring nuclei with $Z=48,52$ and 54 have not yet been seen, their existence is practically certain on the basis of systematics ${ }^{43}$ ). Increased collectivity resulting in even lower excitation energies is indeed expected ${ }^{24}$ ) for $Z>50$ (and $Z<50$ ) as the number of interacting bosons increases. Deformed $\frac{9}{2}^{+}$states with associated rotational bands have been observed ${ }^{43}$ ) in the even- $N$ isotopes with $Z=51,53$, and 55 . These bands appear to be intimitely related to those in the even- $Z$ isotopes. The only difference between the $\frac{9}{2}^{+}$and $0^{+}$states seems to be the proton-hole configuration, $\left(\mathrm{gg}_{\frac{q}{2}}\right)^{-1}$ for odd- $Z$ and a pair of proton holes with about $80 \%\left(0 \mathrm{~g}_{3}\right)^{-2}$ for even- $Z$ while the proton and neutron bosons beyond $Z=50$ and $N=\mathbf{5 0}$ form a highly collective entity of interacting bosons.

It is worth noting that the neutron configuration of the $0^{+}$"pairing vibration" states in the Sn isotopes differs the most from that of the BCS g.s. in the middle of the neutron shell. The ground and "pairing vibration states" become increasingly similar in neutron configuration with decreasing and increasing number of neutrons thus enhancing the possibility for mixing (see subsect. 6.5).

\section{DWBA analysis}

\subsection{GENERAL CONSIDERATIONS}

The data were subjected to various kinds of DWBA analyses. A zero-range analyses (subsect. 5.2) using $\alpha$-cluster wave functions was carried out for all data. The normalization constant $\mathcal{N}$ was derived from a comparison with the $\alpha$-decay of ${ }^{148} \mathrm{Sm}$ (subsect. 5.6). A finite-range analysis (subsect. 5.7) was applied to transitions leading to certain states in ${ }^{118} \mathrm{Sn}$. The dependence on the cluster representation of ${ }^{6} \mathrm{Li}$ and on the $\alpha$-cluster wave function in the heavy nucleus was studied. A semimicroscopic treatment (subsect. 5.8) based on proton pairing and neutron BCS wave functions was performed for all $\mathrm{Te}\left(\mathrm{d},{ }^{6} \mathrm{Li}\right) \mathrm{Sn}$ and $\mathrm{Sn}\left(\mathrm{d},{ }^{6} \mathrm{Li}\right) \mathrm{Cd}$ ground state transitions, for a number of transitions to excited $0^{+}$states as well as to the energetically lowest $2^{+}$and $3^{-}$states in ${ }^{118} \mathrm{Sn}$. The details of the theoretical formulation of the microscopic analysis are presented in the appendices $A$ to $D$.

\subsection{ZERO-RANGE ANALYSIS}

A zero-range DWBA analysis of the data was performed ${ }^{44}$ ) with form factors 
assumed to be simple $\alpha$-cluster wave functions. For $\alpha$-particle pickup reactions on $0^{+}$targets we have ${ }^{6.44}$ )

$$
\begin{gathered}
\frac{\mathrm{d} \sigma_{\exp }(\theta)}{\mathrm{d} \Omega}=\mathcal{N} \frac{S_{\alpha}}{2 J+1} \frac{\mathrm{d} \sigma^{\mathrm{DW}}(\theta)}{\mathrm{d} \Omega}, \\
\gamma_{x}^{2}(s)=\frac{\hbar^{2} s}{2 \mu} S_{\alpha}\left|R^{\mathrm{DW}}(s)\right|^{2}, \\
\theta_{\alpha}^{2}(s)=\gamma_{\alpha}^{2}(s) / \gamma_{w}^{2}(s)=\frac{1}{3} S_{\alpha} s^{3}\left|R^{\mathrm{DW}}(s)\right|^{2}, \\
\gamma_{w}^{2}(s)=3 \hbar^{2} / 2 \mu s^{2} .
\end{gathered}
$$

Here, $S_{\alpha}, \gamma_{\alpha}^{2}(s)$ and $\theta_{\alpha}^{2}(s)$ are the phenomenologically defined $\alpha$-particle spectroscopic factor, reduced width and the dimensionless reduced width, respectively; $\gamma_{w}^{2}(s)$ is the Wigner limit $\left.{ }^{45-48}\right)$. Furthermore, $\mathrm{d} \sigma^{\mathrm{DW}}(\theta) / \mathrm{d} \Omega$ is the DWBA cross section; $\mathscr{N}$ is a normalization factor; $s$ is the channel radius; $\mu$ is the reduced $\alpha$-particle mass; $R^{\mathrm{DW}}(r)$ is the radial part of the normalized $\alpha$-cluster bound state wave function. The spectroscopic factors $S_{\alpha}$ defined by eq. (4) are model-dependent as they depend strongly on the assume $\alpha$-cluster wave function. The channel radius $\mathrm{s}$ is in principle arbitrary. The value used in this work is $s=s_{0} A^{t}\left(s_{0}=1.7 \mathrm{fm}\right)$. This is in the region where the $\alpha$-cluster is picked up, and the reduced widths $\gamma_{\alpha}^{2}(s)$ and $\theta_{a}^{2}(s)$ of eqs. (5) and (6) calculated at this channel radius should be particularly reliable and model independent ${ }^{6}$ ). Note that the dimensionless reduced width $\theta_{a}^{2}(s)$ (for a fixed value of $s_{0}$ ) is roughly proportional to the spectroscopic factor $S_{\alpha}$ as the $A$-dependence of the remaining terms in eq. (3) cancels approximately. Microscopic calculations discussed below in subsect. 5.7 make use of theoretical spectroscopic amplitudes and are, in principle at least, model independent.

The normalization factor $\mathcal{N}$ accounts for the overlap of ${ }^{6} \mathrm{Li}$ with an $\alpha$-particle and $a$ free deuteron ${ }^{49}$ ) and the strength of the effective interaction between the deuteron and $\alpha$-particle, $V_{\mathrm{d} \alpha}$. A finite-range calculation (subsect. 5.6) based on a reliable ${ }^{6} \mathrm{Li}$ cluster wave function would make the normalization $\mathcal{N}$ superfluous in principle.

The significance of the zero-range analysis results from the fact that it accounts for most of the kinematic dependence of the $\left(\mathrm{d},{ }^{6} \mathrm{Li}\right)$ cross sections. Unlike heavy ion reactions only one angular momentum transfer is allowed, $L=J$, in either zero range or finite range calculations. The phenomenological spectroscopic factors $S_{\alpha}$ and reduced widths $\gamma_{a}^{2}$ therefore provide a simple measure for the amount of $\alpha$-particle correlations.

\subsection{OPTICAL-MODEL PARAMETERS}

The deuteron and ${ }^{6} \mathrm{Li}$ optical-model parameters used for $\left(\mathrm{d},{ }^{6} \mathrm{Li}\right)$ are listed in table 13. The deuteron parameter set was obtained by Childs and Daehnick ${ }^{50}$ ) from a 
TABLE 13

Optical-model and bound state parameters

\begin{tabular}{|c|c|c|c|c|c|c|c|c|c|c|c|c|c|c|c|}
\hline Particle & Set & $\begin{array}{c}V \\
(\mathrm{MeV})\end{array}$ & $\begin{array}{c}r_{\mathbf{R}} \\
(\mathbf{f m})\end{array}$ & $\begin{array}{c}a_{\mathrm{R}} \\
(\mathrm{fm})\end{array}$ & $\underset{(\mathrm{MeV})}{V_{\text {s.o. }}}$ & $\begin{array}{l}r_{\mathrm{x.o}} \\
(\mathrm{fm})\end{array}$ & $\begin{array}{l}a_{\mathrm{y.o}} \\
(\mathrm{fm})\end{array}$ & $\begin{array}{c}W \\
(\mathrm{MeV})\end{array}$ & $\begin{array}{c}r_{W} \\
(\mathrm{fm})\end{array}$ & $\begin{array}{c}a_{W} \\
(\mathrm{fm})\end{array}$ & $\begin{array}{c}W^{\prime} \\
(\mathrm{MeV})\end{array}$ & $\underset{(\mathrm{fm})}{r_{W}^{\prime}}$ & $\underset{(\mathrm{fm})}{a_{w}^{\prime}}$ & $\begin{array}{c}r_{\mathrm{c}} \\
(\mathrm{fm})\end{array}$ & Ref. \\
\hline$\left.d^{*}\right)$ & & -92.395 & 1.15 & 0.79 & -5.5 & 1.10 & 0.55 & -1.008 & 1.33 & 0.85 & 12.722 & 1.33 & 0.85 & 1.20 & $\left.{ }^{50}\right)$ \\
\hline${ }^{6} \mathrm{Li}$ & & -240.0 & 1.30 & 0.65 & & & & -16.0 & 1.70 & 0.90 & & & & 1.40 & 51) \\
\hline p & & -55.7 & 1.20 & 0.70 & -12.0 & 1.10 & 0.70 & & & & 11.3 & 1.25 & 0.70 & 1.20 & $\left.{ }^{16}\right)$ \\
\hline t & & -176.0 & 1.14 & 0.72 & & & & -18.0 & 1.61 & 0.82 & & & & 1.14 & 16) \\
\hline$\alpha$-cluster & A & $\left.\sim-145^{b}\right)$ & 1.20 & 0.65 & & & & & & & & & & & \\
\hline$(B=A+x)$ & B & $\left.\sim-128^{b}\right)$ & 1.30 & 0.73 & & & & & & & & & & 1.30 & 52) \\
\hline & C & $\left.\sim-115^{b}\right)$ & 1.40 & 0.65 & & & & & & & & & & & \\
\hline$\alpha$-cluster & $\mathbf{K}$ & $\left.\sim-78^{\circ}\right)$ & 1.508 & 0.65 & & & & & & & & & & 1.508 & $\left.{ }^{49}\right)$ \\
\hline$\left({ }^{6} \mathrm{Li}=\mathrm{d}+x\right)$ & $w$ & $\left.\sim+26^{d}\right)$ & 1.00 & 0.01 & & & & & & & & & & 1.508 & \\
\hline & & $\left.\sim-26^{d}\right)$ & 1.55 & 0.70 & & & & & & & & & & & \\
\hline 2 n cluster & $\mathbf{N}$ & $\left.\sim-73^{c}\right)$ & 1.30 & 0.73 & & & & & & & & & & 1.30 & \\
\hline
\end{tabular}

The analytical form of the optical-model potential is

$$
\begin{aligned}
U_{\mathrm{opt}}(r)=V f\left(r, R_{\mathrm{r}}, a_{\mathrm{r}}\right)+V_{\mathrm{s.0.}} 4 \sigma \cdot l\left(h /\left(m_{\mathrm{x}} c\right)\right)^{2}(1 / r)(\mathrm{d} / \mathrm{d} r) f\left(r, R_{\mathrm{s} .0 .}, a_{\mathrm{s.o.}}\right) \\
+i W f\left(r, R_{W}, a_{W}\right)+i W^{\prime} 4 a_{W}^{\prime}(\mathrm{d} / \mathrm{d} r) f\left(r, R_{W}^{\prime}, a_{W}^{\prime}\right)+V_{\mathrm{c}}\left(r, R_{\mathrm{c}}\right),
\end{aligned}
$$

with

$$
f\left(r, R_{\mathrm{x}}, a_{\mathrm{x}}\right)=\left(1+\exp \left[\left(r-R_{\mathrm{x}}\right) / a_{\mathrm{x}}\right]\right)^{-1}, \quad R_{\mathrm{x}}=r_{\mathrm{x}} A^{1 ; 3} .
$$

2) The parameter set represents potential set $\mathrm{E}$ of Childs and Daehnick ${ }^{50}$ ) for ${ }^{122} \mathrm{Te}+\mathrm{d}$ at $E_{\mathrm{d}}=33 \mathrm{MeV}$.

b) Adjusted to give $\alpha$-particle binding energy; see text. The approximate values listed are for ${ }^{122} \mathrm{Te}={ }^{118} \mathrm{Sn}+\alpha$.

c) Adjusted to give $\alpha$-particle binding energy for ${ }^{6} \mathrm{Li}=\mathrm{d}+\alpha$ of $B_{x}=1.474 \mathrm{MeV}$ with $(N, L)=(1,0)$; see text and ref. ${ }^{49}$ ).

d) Same as footnote $\left.{ }^{c}\right)$ except $(N, L)=(0,0)$; see text.

-) Adjusted to give two-neutron binding energy; see text. The approximate value listed is for ${ }^{118} \mathrm{Sn}={ }^{116} \mathrm{Sn}+(2 \mathrm{n})$.

global fit to scattering and polarization data ( $E_{\mathrm{d}}=11.8$ to $52 \mathrm{MeV}, A=27$ to 232). Various other parameter sets were also employed but are not listed here. The ${ }^{6} \mathrm{Li}$ parameter set was obtained by Chua et al. ${ }^{51}$ ) at $50.6 \mathrm{MeV}$. The proton and triton parameter sets are those used by Fleming et al. ${ }^{16,29}$ ) (see references quoted therein) in their analysis of $(p, t)$ data.

\subsection{BOUND STATE WAVE FUNCTIONS AND QUANTUM NUMBERS}

Simple $\alpha$-cluster wave functions with a specified number of radial nodes bound in a Woods-Saxon plus Coulomb potential well were used as DWBA form factors. The radius and diffuseness parameters of set $B$ in table 13 are those used in the analysis of certain $(\alpha, 2 \alpha)$ reactions ${ }^{52}$ ). The nuclear well depths were adjusted to fit 
the $\alpha$-cluster binding energies. Parameters set B was employed most extensively while sets $\mathrm{A}$ and $\mathrm{C}$ were used only for comparison.

Special techniques for unbound states ${ }^{6}$ ) were used for ${ }^{148} \mathrm{Sm}\left(\mathrm{d},{ }^{6} \mathrm{Li}\right)^{144} \mathrm{Nd}$ which was reanalyzed for normalization purposes.

The quantum numbers for the $\alpha$-cluster wave functions were obtained by using the harmonic oscillator relation

$$
(2 N+L)+(2 n+l)=\sum\left(2 n_{i}+l_{i}\right)
$$

Here, $N, L$ and $n, l$ are the radial and angular momentum quantum numbers for the relative and internal motion of the cluster. The respective quantum numbers for the nucleons which are picked up are $n_{i}$ and $l_{i}$, respectively. Assuming an internal 0 s motion $(n=l=0)$ and specifying the shell-model orbitals of the transferred nucleons, the total number of oscillator quanta $Q=2 N+L$ is uniquely defined. The number of radial nodes $N$ which was used in the calculation are included in tables 1 to 11 . They are based on the simplest shell-model predictions, but it is clear from the semimicroscopic analysis described below that a more realistic description of the form factor must include the coherent superposition of contributions from five or more harmonic oscillator quantum numbers. However, the simple assumptions are still very useful as they allow a consistent comparison between four-nucleon and twonucleon pickup data. Also, the reduced widths $\gamma_{\alpha}^{2}$ and $\theta_{\alpha}^{2}$ extracted from the analysis are expected to be quite reliable since they depend only weakly on the assumed form factor $^{6}$ ).

\subsection{ZERO-RANGE ANALYSIS OF TWO-NEUTRON TRANSFER}

In order to compare $\alpha$-pickup and two-neutron pickup data, it was considered desirable to subject the latter to an analysis essentially identical to that used for $\left(\mathrm{d},{ }^{6} \mathrm{Li}\right)$. The $(\mathrm{p}, \mathrm{t})$ data obtained at $20 \mathrm{MeV}$ by Fleming et al. ${ }^{16,29}$ ) were therefore used in a zero-range DWBA analysis assuming a two-neutron cluster transfer. The $(t, p)$ two-neutron stripping data of Bjerregaard et al. $\left.{ }^{26,}{ }^{27}\right)$ on g.s. $\rightarrow$ g.s. transitions were also subjected to such an analysis. A two-neutron cluster bound in a Woods-Saxon potential well by the two-neutron separation energy $B_{2 n}$ was used as form factor. The number of radial nodes was taken to be that of the assumed neutron component in the $\alpha$-cluster transfer.

\subsection{ALPHA-DECAY AND NORMALIZATION}

The zero-range DWBA normalization factor (see appendix B) deduced from the $\alpha$-cluster wave function B of table 12 is $\mathcal{N}=6.72$. This value together with the normalization factors obtained for the wave functions $A$ and $C$ is included in table 14. As before ${ }^{6}$ ), the value for $\mathcal{N}$ was obtained from the requirement that the 
TABLE 14

Reduced widths $\gamma_{z}^{2}(s)$ at $s=1.7 A_{\text {core }}^{1 / 3}$, spectroscopic factors $S_{x}$ and normalization constants $\mathcal{A}^{\prime \prime}$ for ${ }^{122} \mathrm{Te}\left(\mathrm{d},{ }^{6} \mathrm{Li}\right){ }^{118} \mathrm{Sn}_{\text {e.s. }}$ and ${ }^{148} \mathrm{Sm}\left(\mathrm{d},{ }^{6} \mathrm{Li}\right){ }^{144} \mathrm{Nd}$...

\begin{tabular}{|c|c|c|c|c|c|c|c|c|c|}
\hline \multicolumn{4}{|c|}{ ZRDW } & \multicolumn{3}{|c|}{ FRDW-K } & \multicolumn{3}{|c|}{ FRDW-W } \\
\hline BSWF & A & B & $\mathrm{C}$ & $\mathbf{A}$ & B & C & A & B & $\mathrm{C}$ \\
\hline \multicolumn{10}{|c|}{${ }^{122} \mathrm{Te}\left(d,{ }^{6} \mathrm{~L} i\right)^{118} \mathrm{Sn} \quad E_{\mathrm{d}}=33 \mathrm{MeV}$} \\
\hline $\begin{array}{l}\gamma_{a}^{2}(s) \\
S_{2} \\
\mathcal{L}\end{array}$ & $\begin{array}{l}1010 \\
0.39 \\
2.27\end{array}$ & $\begin{array}{l}405 \\
0.022 \\
6.72\end{array}$ & $\begin{array}{l}490 \\
0.012 \\
4.35\end{array}$ & $\begin{array}{l}\left.3430^{\circ}\right) \\
\left(.3^{\prime \prime)}\right.\end{array}$ & $\begin{array}{l}\left.6430^{2}\right) \\
\left.0.34^{*}\right)\end{array}$ & $\begin{array}{l}\left.9030^{\prime \prime}\right) \\
\left.0.22^{2}\right)\end{array}$ & $\begin{array}{l}7630 \\
2.97\end{array}$ & $\begin{array}{l}6830 \\
0.36 \\
(16.7)\end{array}$ & $\begin{array}{l}6710 \mathrm{eV} \\
0.16\end{array}$ \\
\hline \multicolumn{10}{|c|}{${ }^{148} \mathrm{Sm}\left(d,{ }^{6} \mathrm{Li}\right)^{144} \mathrm{Nd} \quad E_{\mathrm{d}}=35 \mathrm{MeV}$} \\
\hline $\begin{array}{l}\gamma_{a}^{2}(s) \\
S_{a} \\
\mathcal{H}\end{array}$ & $\begin{array}{l}\left.1070^{b \cdot c}\right) \\
\left.0.60^{b \cdot c}\right) \\
\left.2.27^{b}\right)\end{array}$ & $\begin{array}{l}\left.785^{\circ}\right) \\
\left.0.053^{\circ}\right) \\
6.72\end{array}$ & $\begin{array}{l}\left.725^{\mathrm{c}}\right) \\
\left.0.021^{\mathrm{c}}\right) \\
4.35\end{array}$ & $\begin{array}{l}\left.2960^{\circ}\right) \\
\left.1.66^{\circ}\right)\end{array}$ & $\begin{array}{l}\left.12370^{\circ}\right) \\
\left.0.83^{\circ}\right)\end{array}$ & $\begin{array}{l}\left.21810^{\circ}\right) \\
\left.0.64^{4}\right)\end{array}$ & $\begin{array}{l}\left.14140^{b}\right) \\
\left.7.94^{b}\right)\end{array}$ & $\begin{array}{l}13160 \\
0.88 \\
(16.7)\end{array}$ & $\begin{array}{l}11310 \mathrm{eV} \\
0.33\end{array}$ \\
\hline
\end{tabular}

The nine zero-range and finite-range DWBA calculations correspond to those of figs. 6 and 7.

") Fair agreement between experimental and calculated angular distributions.

b) Poor agreement between experimental and calculated angular distributions.

c) Normalized to the $\alpha$-decay ${ }^{148} \mathrm{Sm} \rightarrow{ }^{144} \mathrm{Nd}=\alpha$.

reduced width $\gamma_{\alpha}^{2}$ at a suitably chosen channel radius s determined from the known $\alpha$-decay lifetime of ${ }^{148} \mathrm{Sm}$ via

$$
\Gamma_{\alpha}=\hbar \lambda_{\alpha}=2 \gamma_{\alpha}^{2}(s) P_{L}\left(Q_{\alpha}, s\right)
$$

is equal to that from the ${ }^{148} \mathrm{Sm}\left(\mathrm{d},{ }^{6} \mathrm{Li}\right)$ reaction via eqs. (4) and (5). Here,

$$
P_{L}\left(Q_{\alpha}, s\right)=\lim _{r \rightarrow \infty}\left[k s\left|\frac{r R_{L}(r)}{s R_{L}(s)}\right|^{2}\right]
$$

is the penetrability calculated from the same $\alpha$-cluster wave function B. The spectroscopic factors $S_{\alpha}$ and reduced widths $\gamma_{\alpha}^{2}$ and $\theta_{\alpha}^{2}$ of tables 1 to 11 are therefore "absolute". The quantity $S_{\alpha}$ is, of course, still phenomenologically defined and model-dependent. A method for describing both $\alpha$-decay and $\alpha$-cluster transfer reactions reported to be independent of channel radii and $R$-matrix theory has been introduced recently by Jackson and Rhoades-Brown ${ }^{53,54}$ ).

\subsection{FINITE-RANGE ANALYSIS AND CLUSTER REPRESENTATION OF ${ }^{6} \mathrm{Li}$}

A finite-range DWBA analysis ${ }^{44}$ ) was carried out for ${ }^{122} \mathrm{Te}\left(\mathrm{d},{ }^{6} \mathrm{Li}\right)$ transitions to a number of states in ${ }^{118} \mathrm{Sn}$ as well as for the ${ }^{148} \mathrm{Sm}\left(\mathrm{d},{ }^{6} \mathrm{Li}\right){ }^{144} \mathrm{Nd}$ ground state transitions. The cross sections for $\alpha$-particle pickup are given by

$$
\frac{\mathrm{d} \sigma_{\text {exp }}(\theta)}{\mathrm{d} \Omega}=S_{\alpha}^{\prime} S_{\alpha} \frac{\mathrm{d} \sigma^{\mathrm{DW}}(\theta)}{\mathrm{d} \Omega},
$$

where $S_{\alpha}$ is again the spectroscopic factor for the target. The quantity $S_{\alpha}^{\prime}$ describes 


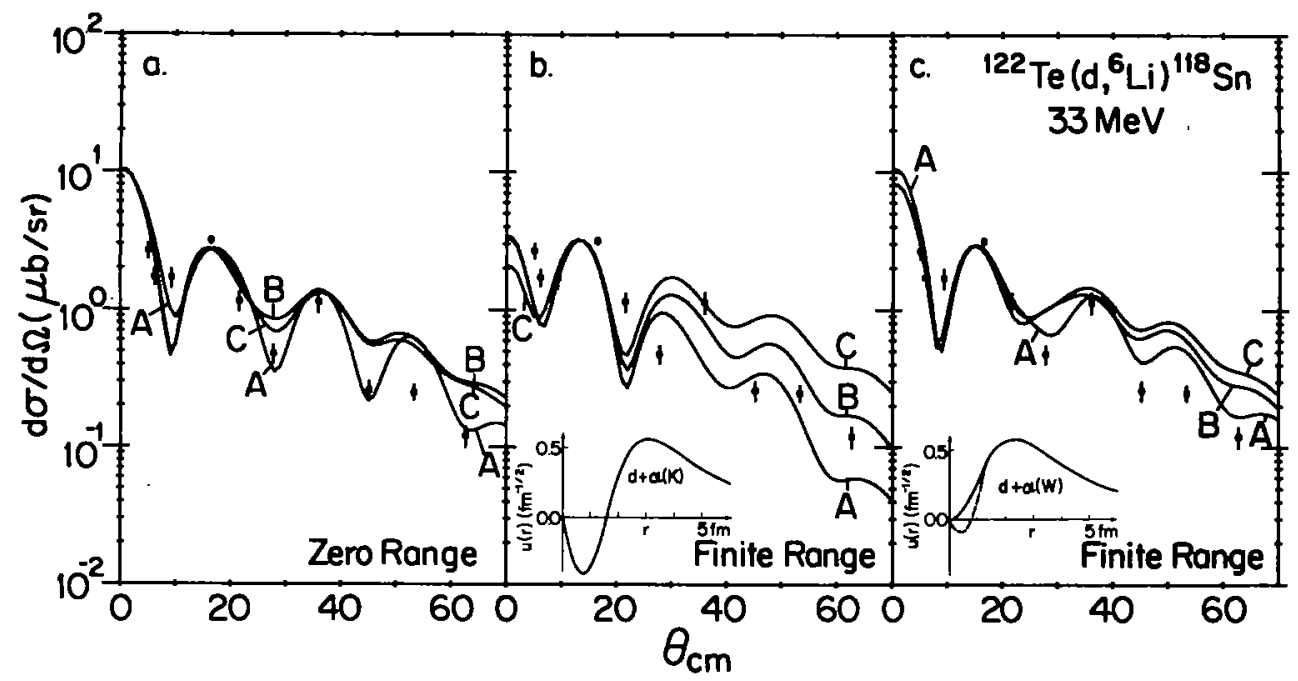

Fig. 6. Experimental and calculated angular distributions for ${ }^{122} \mathrm{Te}\left(\mathrm{d},{ }^{6} \mathrm{Li}\right){ }^{18} \mathrm{Sn}_{\text {,... }}$ at $E_{\mathrm{d}}=33 \mathrm{MeV}$. The DWBA calculations are based on (a) zero-range; (b) finite-range with $V_{\mathrm{du}}$ potential $K$; (c) finite range with $V_{\mathrm{d} x}$ potential $\mathrm{W}$. The calculation are performed with different $\alpha$-cluster potentials: (A) $r_{0}=1.2 \mathrm{fm}, a=0.65 \mathrm{fm}$; (B) $r_{0}=1.3 \mathrm{fm}, a=0.73 \mathrm{fm}$; (C) $r_{0}=1.4 \mathrm{fm}, a=0.65 \mathrm{fm}$. Compare figs. 6 and 7 with table 14 .

the spectroscopic overlap of ${ }^{6} \mathrm{Li}$ and $\mathrm{d}+\alpha$. Shell-model calculations ${ }^{55-57}$ ) assign to it a value $S_{\alpha}^{\prime} \lessgtr 1.0$. Reduced widths $\gamma_{\alpha}^{2}(s)$ and dimensionless reduced widths $\theta_{\alpha}^{2}(s)$ were obtained from $S_{\alpha}$ and eqs. (5) and (6). Finite range calculations are sensitive not only to the $\alpha$-cluster wave function in the heavy particle, but also to the relative $\alpha$ cluster wave function in ${ }^{6} \mathrm{Li}$. The parameters for two such cluster representations are included in table 13 . The effective deuteron $\alpha$-particle potential $V_{\mathrm{d} \alpha}$ denoted $K$ has been discussed by Kubo and Hirata ${ }^{49}$ ). It generates a 1s wave function (one radial node) and it is shown graphically as an inset in fig. 6. One of several other potentials $V_{\mathrm{dz}}$ which was used is the one denoted $W$. It includes a soft core which appears to be important ${ }^{36,57}$ ) to account for antisymmetrization in the relative motion of the deuteron and $\alpha$-particle. The wave function at small relative distances is reduced, and the $1 \mathrm{~s}$ wave function (dashed line in the inset of fig. 7) is approximated for use in this study by a 0 s wave function (solid line). The wave function is similar to one used by Watson et al. ${ }^{56}$ ) in a study of $\alpha$-particle knockout from ${ }^{6} \mathrm{Li}$.

The deuteron and ${ }^{6} \mathrm{Li}$ optical-model parameters used in the finite-range analysis are again those of table 13.

\subsection{ANALYSIS WITH $\alpha$-PARTICLE SPECTROSCOPIC AMPLITUDES FROM SHELL-MODEL WAVE FUNCTIONS AND MICROSCOPIC FORM FACTORS}

Microscopic and macroscopic analyses differ in two main respects. The nuclear plus Coulomb interaction $V_{\mathrm{d} z}$ between the centers of masses of the deuteron projectile 


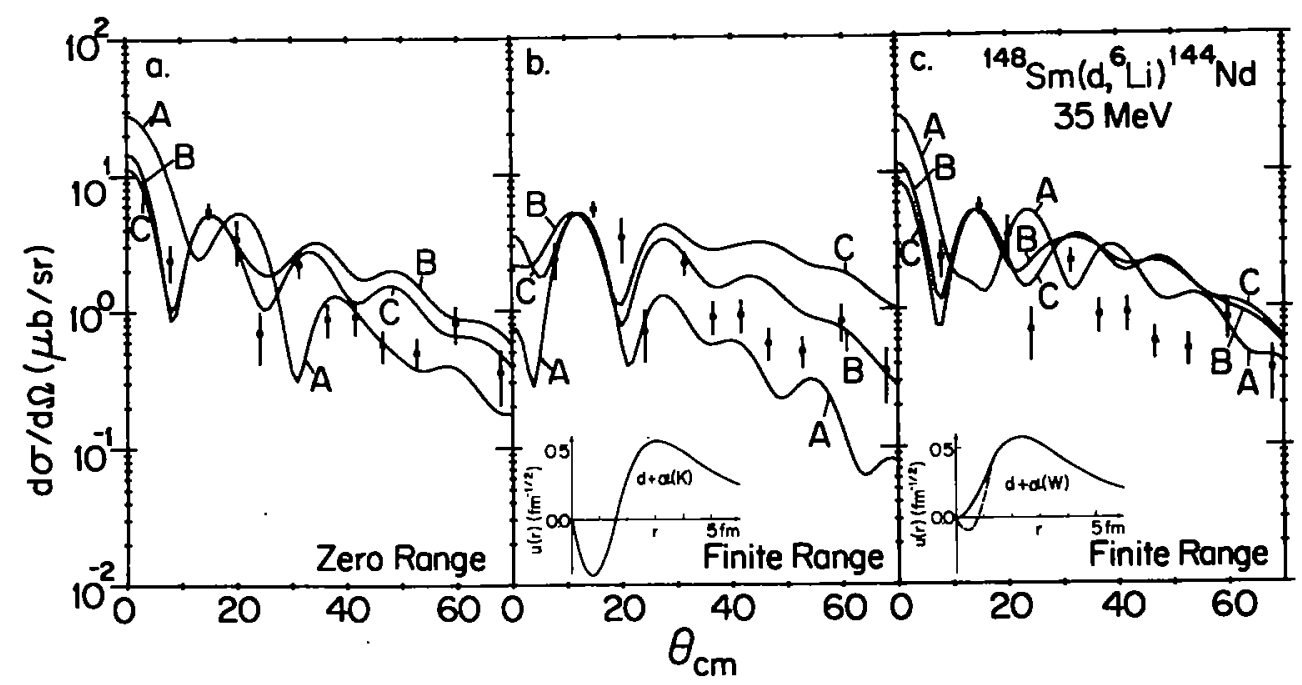

Fig. 7. Experimental (ref. $\left.{ }^{6}\right)$ ) and calculated angular distributions for ${ }^{148} \mathrm{Sm}\left(\mathrm{d},{ }^{6} \mathrm{Li}\right)^{144} \mathrm{Nd}$ at $E_{\mathrm{d}}=35 \mathrm{MeV}$. See caption for fig. 6 for details.

and the four nucleons constituting the $\alpha$-cluster which is picked up must be replaced by the sum of the two times four interactions between individual nucleons. This is a formidable task, and even in two-nucleon transfer reactions a description using a microscopic interaction has been used only occasionally, mostly in heavy-ion reactions ${ }^{58-63}$ ). No attempts in this respect will be made here.

The other important difference between the macroscopic and microscopic analysis relates to the nuclear wave functions. The phenomenological cluster wave functions which are assumed in a macroscopic treatment have to be replaced by more realistic microscopic cluster wave functions which can, for example, be constructed from shell-model configurations. Such procedures are well established for two-nucleon transfer reactions ${ }^{64-69}$ ) where it is assumed in first order that the transition proceeds by the transfer of a nucleon pair without rearrangement of the core.

Kurath and Towner ${ }^{9}$ ) have developed a procedure for expressing $\alpha$-particle spectroscopic amplitudes as a coherent sum of two-proton and two-neutron spectroscopic amplitudes. The availability of theoretical two-nucleon spectroscopic amplitudes in the Sn region (appendix D) makes it possible to apply this formalism to the present data.

The equations used in this work are essentially equivalent to eqs. (5) to (8) and eq. (21) of ref. $\left.{ }^{9}\right)$ specialized to the $\alpha$-particle pickup reaction $B\left(d,{ }^{6} \mathrm{Li}\right) \mathrm{A}$ with $\mathrm{B}=$ $\mathrm{A}+\alpha$ and ${ }^{6} \mathrm{Li}=\mathrm{d}+\alpha$. They are presented in appendices $\mathrm{A}$ and $\mathrm{B}$. Although some simplifications have been included, the more general equations can be reintroduced if the availability of the relevant structure information warrants it or if more general procedures for constructing form factors are considered.

The microscopic form factors in this work are assumed to depend only on the 
angular momentum transfer $L, M$ and the total number of harmonic oscillator quanta $Q$ but not on the quantum numbers of the individual nucleons. (See appendix $A$ and fig. 12 for notation.) The depth of a Woods-Saxon plus Coulomb potential well for each value of $Q$ is adjusted to give the correct $\alpha$-particle binding energy $B_{\alpha}$. More sophisticated methods for generating microscopic form factors are of course possible as extensions of methods used in two-nucleon transfer. Such methods might include the use of harmonic oscillator wave functions ${ }^{64,66}$ ) with properly chosen size parameters, the tail region matched to a Coulomb function, or the use of Woods-Saxon single-particle wave functions ${ }^{65}$ ) bound at properly chosen fractions of the $\alpha$-particle separation energy. However, it was found in the present analysis that the calculated cross sections are almost independent of the $\alpha$-cluster wave function in the interior as the pickup takes place mostly in the tail region. The main effect of the microscopic form factor is therefore to provide the proper normalization for the important exterior region. This provides a partial justification for the above assumption. Instead of about 60 different form factors for transitions to $0^{+}$states and 200 to 300 for $J^{x} \neq 0$ states, with the simplified procedure only about five form factors are required for each transition as all contributions with the same value of $Q$ are combined.

The general spectroscopic and kinematic equations presented in the appendices $A$ and $B$ do not include the effects introduced by the change in size of the $\alpha$-cluster before and after the transfer. However, the ensuing reductions in cross sections have been estimated and the results are included in appendix $\mathbf{C}$.

The two-nucleon spectroscopic amplitudes needed for the calculations of the microscopic $\alpha$-cluster spectroscopic amplitudes are discussed in appendix $D$. The two-neutron amplitudes were obtained ${ }^{18}$ ) from BCS wave functions for the $\mathrm{Sn}$ isotopes. The two-proton amplitudes were obtained ${ }^{13,20}$ ) from the addition and removal phonon amplitudes calculated with an assumed magic proton number $Z=50$.

\section{Discussion}

\subsection{ZERO-RANGE AND FINITE-RANGE DWBA ANALYSIS}

Transitions to two $0^{+}$ground states were selected for a more detailed study of the appropriate $\alpha$-cluster wave function and ${ }^{6} \mathrm{Li}$ cluster representation for use in zero-range and finite-range analyses. These are ${ }^{122} \mathrm{Te}\left(\mathrm{d},{ }^{6} \mathrm{Li}\right){ }^{118} \mathrm{Sn}_{\text {g.s. }}$ and ${ }^{148} \mathrm{Sm}(\mathrm{d}$, $\left.{ }^{6} \mathrm{Li}\right)^{144} \mathrm{Nd}_{\text {g.s. }}$. The latter reaction was studied previously ${ }^{6}$ ) at $E_{\mathrm{d}}=35 \mathrm{MeV}$. It was included because it provides a convenient calibration for absolute spectroscopic factors and reduced widths since ${ }^{148} \mathrm{Sm}$ is a long-lived $\alpha$-emitter with known $\alpha$-decay rate $^{70}$ ).

Figs. 6 and 7 display the experimental and calculated angular distributions for the two reactions. They were obtained using (a) zero-range DWBA, (b) finite-range 
DWBA, $V_{\mathrm{d} \alpha}$ potential $K$ with $(N, L)=(1,0)$, and (c) finite-range DWBA, $V_{\mathrm{d} \alpha}$ potential $W$ with $(N, L)=(0,0)$. The respective wave functions for the relative $\mathrm{d}-\alpha$ motion are shown as insets (see also subsect. 5.7). The curves labeled A, B and C are for $\alpha$ cluster bound states with radius parameters $r_{0}=1.2,1.3$ and $1.4 \mathrm{fm}$ (potentials $A, B$ and $C$ of table 13 ). The $\alpha$-cluster wave function for ${ }^{144} \mathrm{Nd}+\alpha$ is only quasibound. It was constructed ${ }^{6}$ ) by normalizing the wave function within a cutoff radius of $20 \mathrm{fm}$. At this radius the $\alpha$-cluster wave function is about 10 orders of magnitude smaller than in the interior.

All angular distributions of figs. 3, 4,6 and 7 were obtained with the optical model parameter sets of table 13 . Several other sets were tried and found to have only relatively small effects on the calculated distributions with changes in spectroscopic strength of about $\pm 30 \%$. Neglecting the spin-orbit term in the deuteron potential changes the cross section only little but leads to more pronounced maxima and minima at larger angles. Neglecting the Coulomb term in the effective interaction $V_{\text {da }}$ leads to a $30 \%$ increase. However, the calculated distributions change considerably with the assumed form factors, and the agreement with the data is strongly effected. All zero-range curves agree very well except curve $A$ for ${ }^{148} \mathrm{Sm}$. The good agreement between data and zero-range calculations appears to be a more general feature of $\left(\mathrm{d},{ }^{6} \mathrm{Li}\right)$ reactions. The reasons are unclear as the transfer of a point-like $\alpha$-particle is not a viable assumption. Finite range calculations with the $V_{\mathrm{d} \alpha}$ potential $K$ of Kubo and Hirata ${ }^{49}$ ) and one radial node in the relative $d-\alpha$ motion give a first maximum at an angle which is too small, and the second maximum is out of phase. Similar results were obtained ${ }^{71}$ ) for ${ }^{208} \mathrm{~Pb}\left(\mathrm{~d},{ }^{6} \mathrm{Li}\right){ }^{204} \mathrm{Hg}$ at higher energy. Good agreement with the data and with zero-range calculations is obtained (not shown) for potential $\mathrm{K}$ and no radial node. The finite-range calculations with the $V_{\mathrm{d} \alpha}$ potential $\mathrm{W}$ containing a soft core are in reasonable agreement with the data except again for curve $A$ for ${ }^{148} \mathrm{Sm}$. Potentials $B$ and $W$ of table 13 were chosen as standard sets for all other transitions.

Reduced widths $\gamma_{\alpha}^{2}(s)$ and spectroscopic factors $S_{\alpha}$ were extracted for all angular distributions of figs. 6 and 7 and compared to the values deduced from the $\alpha$-decay of ${ }^{148} \mathrm{Sm}$. The results are displayed in table 14. The values of $\gamma_{\alpha}^{2}(s)$ and $S_{\alpha}$ listed for ${ }^{148} \mathrm{Sm}(\mathrm{ZRDW})$ were obtained from eq. (9) and the known half-life ${ }^{70}$ ) of $T_{t}=$ $(8 \pm 2) \times 10^{15}$ y. The penetrabilities $P_{0}\left(Q_{a}, s\right)$ at the channel radius $s=1.7 A^{t^{2}} \mathrm{fm}$ depend only weakly on the assumed geometry $\left(\mathrm{A}: P_{0}=0.85 \times 10^{-42} ; \mathrm{B}: P_{0}=\right.$ $1.15 \times 10^{-42} ; \mathrm{C}: P_{0}=1.25 \times 10^{-42}$ ) leading to slightly different reduced widths $\gamma_{\alpha}^{2}(s)$. As is well known ${ }^{6}$ ), spectroscopic factors are far more sensitive to the various parameters resulting in variations by a factor of 30 . The normalization constants $\mathscr{N}$ are obtained from the requirement that reaction and decay yield the same width. As mentioned before, an added uncertainty of $\pm 30 \%$ in these values results from the dependence on optical model parameters.

The zero-range reduced widths and spectroscopic factors for ${ }^{122} \mathrm{Te}$ were obtained with the above normalization constants. They are absolute as they are normalized 
to $\alpha$-decay. Again, contrary to the reduced widths, the strong dependence of spectroscopic factors on the geometry of the bound state wave function is evident.

The reduced widths obtained from the finite-range analyses should, in principle, be absolute and therefore agree with those from the $\alpha$-decay of ${ }^{148} \mathrm{Sm}$ and the normalized zero-range analysis. Instead, they are a factor of $\sim 16$ larger indicating that finite-range calculations underestimate the cross section considerably. The reasons are not clear but may be related to the relative motion wave function for the $\alpha+d$ component of the ${ }^{6} \mathrm{Li}$ ground state wave function. It is hoped new data on heavier radioactive targets $\left({ }^{238} \mathrm{U}\right.$ and ${ }^{232} \mathrm{Th}$; ref. $\left.{ }^{15}\right)$ may help to resolve this problem. Similar problems have been encountered in the analysis of $\left({ }^{16} \mathrm{O},{ }^{12} \mathrm{C}\right)$ data $\left.{ }^{72}\right)$. Some of the observed discrepancy may result from contributions from two-step processes which are important in two-nucleon transfer ${ }^{73-75}$ ). However, the systematic observation of highly selective $l=0$ transitions ${ }^{3}$ ) suggests that sequential nucleon transfer is not likely to be dominant in $\left(\alpha,{ }^{6} \mathrm{Li}\right)$.

The spectroscopic factors for the finite range analysis are again strongly dependent on the bound state wave function but they do not change much with the ${ }^{6} \mathrm{Li}$ cluster representation. The reduced width for the $V_{d \alpha}$ potential $W$ are quite stable, and the bound state wave function $B$ leads to a ratio of 16.7 between the respective finiterange and normalized zero-range calculations. This value can be introduced as an ad hoc normalization (given in parenthesis in table 14) which will normalize the finiterange calculations to the $\alpha$-decay of ${ }^{148} \mathrm{Sm}$.

\subsection{ANGULAR DISTRIBUTIONS, REDUCED WIDTHS AND SPECTROSCOPIC FACTORS}

The experimental and calculated angular distributions for ${ }^{122} \mathrm{Te}\left(\mathrm{d},{ }^{6} \mathrm{Li}\right){ }^{18} \mathrm{Sn}$ are displayed in figs. 3 and 4 . Several states could not be resolved, and the comparisons are therefore made with the incoherent superposition of two calculated distributions. The agreement is quite good, both for the zero-range calculations (thin lines) and the finite-range calculations (thick lines).

The spectroscopic factors $S_{a}$, reduced widths $\gamma_{a}^{2}(s)$ and dimensionless reduced widths $\theta_{a}^{2}(s)$ obtained from ZRDW for these transitions as well as for the more limited data from all other targets are included in tables 1 to 11 . The number of assumed radial nodes $N$ in the $\alpha$-cluster bound state wave function is indicated. When normalized independently to the $\alpha$-decay of ${ }^{148} \mathrm{Sm}$, zero-range and finite-range spectroscopic factors are in excellent agreement, with the latter on the average about $10 \%$ larger. Table 1 includes $S_{\alpha}$ from FRDW for a few selected transitions in parenthesis.

\subsection{COMPARISON BETWEEN (d, $\left.{ }^{6} \mathrm{Li}\right)$ AND (p, t) REACTIONS}

As already mentioned in subsect. 3.1 there exist basic differences between the spectra observed in $\alpha$-cluster and two-neutron pickup. These differences concern mostly 
the strength of transitions to excited states relative to the ground states strengths. The effects can be quantified by comparing spectroscopic factors.

Fig. 8 displays spectroscopic factors $S_{\alpha}$ from tables 1 to 11 for Te(d, $\left.{ }^{6} \mathrm{Li}\right) \mathrm{Sn}$ and $\operatorname{Sn}\left(\mathrm{d},{ }^{6} \mathrm{Li}\right) \mathrm{Cd}$. The final states are essentially the $0^{+}, 2^{+}, 4^{+}, 3^{-}, 5^{-}$and $7^{-}$states included in fig. 5. Results for two $0^{+}$states are shown, the ground states and the $0_{2}^{+}$ states which carry proton pairing vibration strength $\left.{ }^{17}\right)$ as seen in $\left({ }^{3} \mathrm{He}, \mathrm{n}\right)$. A $2_{2}^{+}$ state is included for two $\mathrm{Sn}$ isotopes. These are possible candidates for quadrupole proton pairing vibration strength although the state in ${ }^{118} \mathrm{Sn}$ has also been identified $^{42}$ ) as a member of a rotational band based on the $0_{2}^{+}$state.

Fig. 8 includes the spectroscopic strength for ${ }^{A} \operatorname{Sn}\left(\mathrm{p}, \mathrm{t}^{A-2} \mathrm{Sn}\right.$ reactions extracted from the data of Fleming $e t a l .{ }^{16,29}$ ) as described in subsect. 5.5. The results are

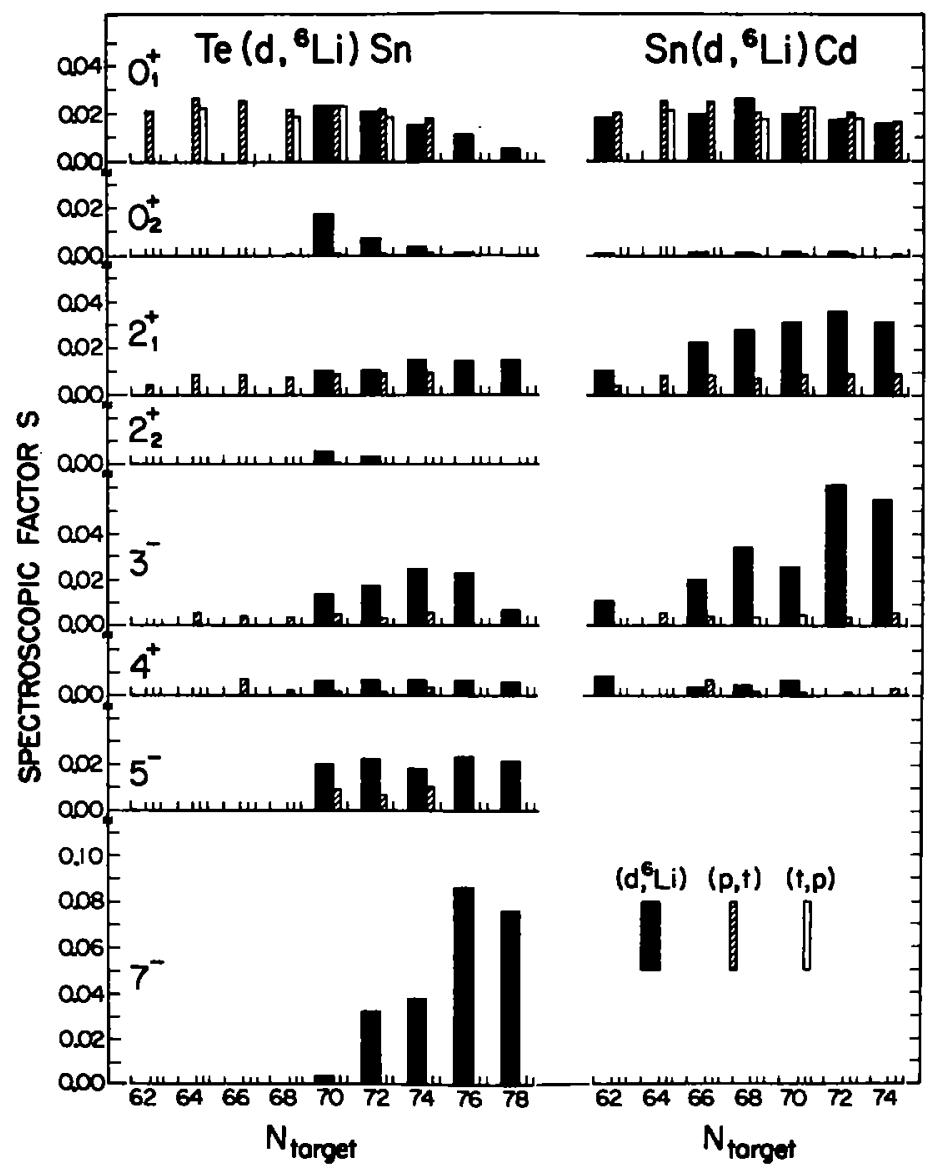

Fig. 8. Absolute $\alpha$-particle spectroscopic factors $S_{\alpha}$ for the $\left(d,{ }^{6} \mathrm{Li}\right)$ reactions on even $\mathrm{Te}$ and $\mathrm{Sn}$ isotopes and comparison with relative spectroscopic factors $S_{2 n}$ from $(p, t)$ and $(t, p)$ (ground states only) reactions involving the transfer of the same neutron pairs. The latter are normalized to $S_{a}$ for ${ }^{122} \mathrm{Te}\left(\mathrm{d},{ }^{6} \mathrm{Li}\right)^{118} \mathrm{Sn}$. 
combined with both $\mathrm{Te}\left(\mathrm{d},{ }^{6} \mathrm{Li}\right)$ and $\mathrm{Sn}\left(\mathrm{d},{ }^{6} \mathrm{Li}\right)$ such that they involve the transfer of the same neutron pair. The strengths for ${ }^{120} \mathrm{Sn}(\mathrm{p}, \mathrm{t})^{118} \mathrm{Sn}$ and its inverse reaction have been arbitrarily normalized to that for ${ }^{122} \mathrm{Te}\left(\mathrm{d},{ }^{6} \mathrm{Li}\right){ }^{118} \mathrm{Sn}$. The $(\mathrm{t}, \mathrm{p})$ data of Bjerregaard et al. ${ }^{26.27}$ ) on $\mathrm{Sn}$ targets have been included in the same manner but only for transitions to ground state as detailed balance demands identical results for the corresponding $(p, t)$ and $(t, p)$ transitions.

The dependence on neutron number is practically identical for the ground state. It thus appears that the two protons transferred in $\left(\mathrm{d},{ }^{6} \mathrm{Li}\right)$ act essentially as spectators with no drastic changes in their configuration. Such a close correspondence between $\alpha$-cluster and two-nucleon transfer has been observed experimentally before ${ }^{3-7}$ ). It supports the theory that $\alpha$-particle spectroscopic amplitudes can be expressed ${ }^{9-13}$ ) as a coherent sum of two-neutron and two-proton spectroscopic amplitudes (see eqs. (24)-(27) of appendix A). For nuclei with strong pairing correlations in the ground state the coherent sum can approximately be factorized into neutron and proton components. Betts ${ }^{10,11}$ ) has developed this approximation with particular emphasis on pairing vibration states.

The relative spectroscopic strength for two-nucleon transfer to excited states is always smaller, in most cases considerably smaller, than the respective four-nucleon transfer strength. The transition to the $0_{2}^{+}$state in ${ }^{118} \mathrm{Sn}$, for example, is essentially forbidden. It has a strong proton pairing vibration component as indicated by $\left({ }^{3} \mathrm{He}, \mathrm{n}\right)$. In $\alpha$-transfer, on the other hand, it is possible to populate states with proton excitations, neutron excitations, or both.

Another example is the increased strength seen in $\left(\mathrm{d},{ }^{6} \mathrm{Li}\right)$ for transitions to states $J \neq 0$. This can be explained as resulting from the coherent superposition of contributions where a proton pair is transferred with $J_{n}=0$ or $J_{n}=J$ and a neutron pair with $J_{v}=J$ or $J_{v}=0$. Other combinations may also contribute, but usually much less. Only contributions with $J_{v}=J$ are, of course, allowed in two-neutron transfer. The relative spectroscopic strength of $\left(d,{ }^{6} \mathrm{Li}\right)$ and $(\mathrm{p}, \mathrm{t})$ thus reflects upon the neutron and proton excitations in the respective state. A more detailed discussion of the absolute $\left(\mathrm{d},{ }^{6} \mathrm{Li}\right)$ strengths in terms of microscopic wave functions will be presented in subsects. 6.4 and 6.6 .

It appears that very little or no investigations of $n p$ transfer reactions have been performed in the Sn region. Possible correlations between $\alpha$-transfer and np transfer can therefore not be studied.

It is in principle possible in $\alpha$-cluster transfer to observe states which cannot be seen in both two-neutron and two-proton transfer. For example, neutron and proton pairing vibration states can be excited in $(t, p)$ and $\left({ }^{3} \mathrm{He}, n\right)$, respectively. Both of these can be excited in $\left({ }^{6} \mathrm{Li}, \mathrm{d}\right)$, but only the $\alpha$-transfer may excite additional $4 \mathrm{p}-4 \mathrm{~h}$ states which involve neutron and proton pair excitations simultaneously, often referred to as $\alpha$-cluster or $\alpha$-vibrational states. No such states could be identified in the present work. 


\subsection{SEMI-MICROSCOPIC ANALYSIS WITH BCS WAVE FUNCTIONS}

Fig. 9 and table 15 display the results of semi-microscopic calculations based on $\alpha$-particle spectroscopic amplitudes from shell-model wave functions. The bar diagram fig. 9 represents a comparison between experimental and calculated cross sections for the $\mathrm{Te}$ and $\mathrm{Sn}$ targets. All g.s. transitions are displayed (including $114,126,128 \mathrm{Sn}$ ) as well as the transitions to the $0^{+}$proton pairing vibration states in the $\mathrm{Sn}$ isotopes. Table 15 shows a similar comparison for ${ }^{122} \mathrm{Te}\left(\mathrm{d},{ }^{6} \mathrm{Li}\right)$ and ${ }^{120} \mathrm{Sn}\left(\mathrm{d},{ }^{6} \mathrm{Li}\right)$ including transitions to states in ${ }^{118} \mathrm{Sn}$ with $J^{\pi} \neq 0$ and to another $0^{+}$state at $2057 \mathrm{keV}$, presumably a neutron two-quasiparticle state.

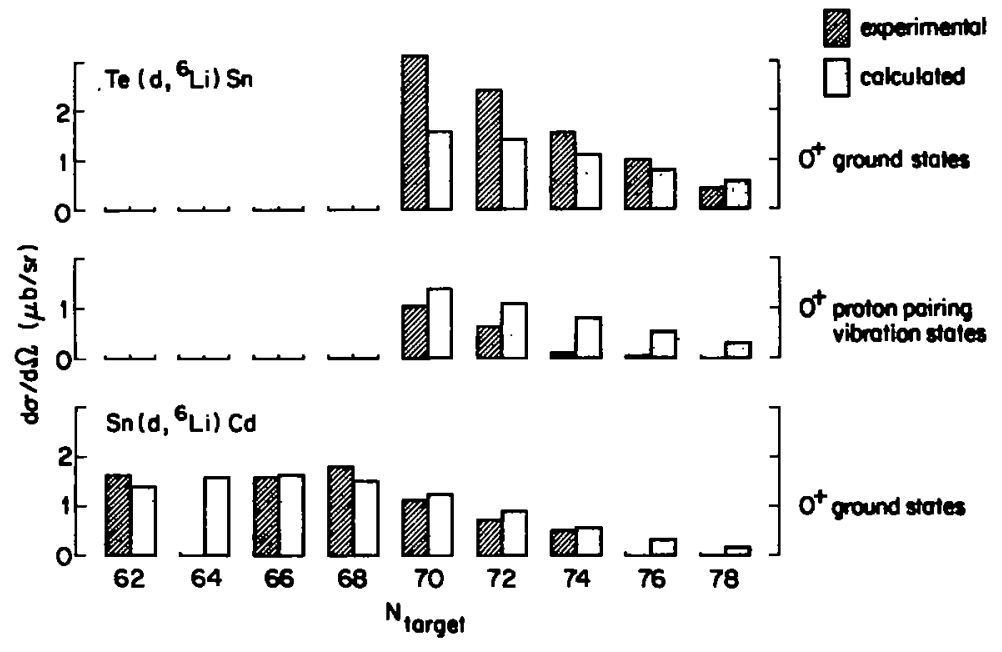

Fig. 9. Comparison of experimental and calculated cross sections for Te(d, $\left.{ }^{6} \mathrm{Li}\right) \mathrm{Sn}$ and $\mathrm{Sn}\left(\mathrm{d},{ }^{6} \mathrm{Li}\right) \mathrm{Cd}$ at $\theta_{\mathrm{lnb}}=16^{\circ}$ and $E_{\mathrm{d}}=33 \mathrm{MeV}$. The calculated values are from semi-microscopic calculations as described in the text normalized to the $\alpha$-decay ${ }^{148} \mathrm{Sm} \rightarrow{ }^{144} \mathrm{Nd}+\alpha$.

TABLE 15

Experimental and calculated corss sections for ${ }^{122} \mathrm{Te}\left(\mathrm{d},{ }^{6} \mathrm{Li}\right){ }^{118} \mathrm{Sn}$ and ${ }^{120} \mathrm{Sn}\left(\mathrm{d},{ }^{6} \mathrm{Li}\right){ }^{116} \mathrm{Cd}$

\begin{tabular}{|c|c|c|c|c|c|c|}
\hline Target & $E_{\mathrm{x}}(\mathrm{keV})$ & $J^{x}$ & $0_{\text {c.m.m. }}$ & $\begin{array}{c}(\mathrm{d} \sigma / \mathrm{d} \Omega)(\exp ) \\
(\mu \mathrm{b} / \mathrm{sr})\end{array}$ & $\begin{array}{c}(\mathrm{d} \sigma / \mathrm{d} \Omega)(\mathrm{calc}) \\
(\mu \mathrm{b} / \mathrm{sr})\end{array}$ & $\varepsilon$ \\
\hline${ }^{122} \mathrm{Te}$ & 0000 & $0^{+}$ & .16 & 3.17 & 1.57 & 2.02 \\
\hline${ }^{122} \mathrm{Te}$ & 1230 & $2^{+}$ & 21 & 0.40 & 0.71 & 0.57 \\
\hline${ }^{122} \mathrm{Te}$ & 1758 & $0^{+}$ & 16 & 1.32 & 1.40 & 0.95 \\
\hline${ }^{122} \mathrm{Te}$ & 2057 & $0^{+}$ & 16 & 0.10 & 0.19 & 0.53 \\
\hline${ }^{122} \mathrm{Te}$ & 2310 & $3^{-}$ & 13 & 0.45 & 2.16 & 0.21 \\
\hline${ }^{120} \mathrm{Sn}$ & 0000 & $0^{+}$ & 16 & 1.13 & 1.24 & 0.91 \\
\hline
\end{tabular}

The calculated values are from microscopic calculations as described in the text normalized to the $\alpha$-decay ${ }^{148} \mathrm{Sm} \rightarrow{ }^{144} \mathrm{Nd}+\alpha$. The enhancement factor $\varepsilon$ is defined as the ratio of experimental and calculated cross section. 
TABLE 16

Alpha-particle spectroscopic amplitudes $A_{1}^{Q L}$ for $\mathrm{B}\left(\mathrm{d},{ }^{6} \mathrm{Li}\right) \mathrm{A}$ reactions leading to ground and excited $0^{+}$ states as a function of the harmonic oscillator quantum number $Q$

\begin{tabular}{|c|c|c|c|c|c|c|c|c|}
\hline \multirow{2}{*}{ Reaction } & \multirow{2}{*}{$E_{\mathrm{x}}(\mathrm{keV})$} & \multirow{2}{*}{$J^{\pi}$} & \multicolumn{6}{|c|}{$100 A_{1}^{Q L}$} \\
\hline & & & $Q=12$ & $Q=14$ & $Q=16$ & $Q=18$ & $Q=20$ & $Q=22$ \\
\hline${ }^{122} \mathrm{Te}\left(\mathrm{d},{ }^{6} \mathrm{Li}\right){ }^{118} \mathrm{Sn}$ & 0000 & $0^{+}$ & 0.000 & 2.505 & 5.498 & 2.950 & 0.157 & 0.004 \\
\hline${ }^{124} \mathrm{Te}\left(\mathrm{d},{ }^{6} \mathrm{Li}\right){ }^{120} \mathrm{Sn}$ & 0000 & $0^{+}$ & 0.000 & 2.367 & 4.901 & 3.140 & 0.170 & 0.005 \\
\hline${ }^{126} \mathrm{Te}\left(\mathrm{d},{ }^{6} \mathrm{Li}\right)^{122} \mathrm{Sn}$ & 0000 & $0^{+}$ & 0.000 & 2.146 & 4.189 & 3.039 & 0.163 & 0.005 \\
\hline${ }^{128} \mathrm{Te}\left(\mathrm{d},{ }^{6} \mathrm{Li}\right){ }^{124} \mathrm{Sn}$ & 0000 & $0^{+}$ & 0.000 & 1.893 & 3.506 & 2.865 & 0.154 & 0.005 \\
\hline${ }^{130} \mathrm{Te}\left(\mathrm{d},{ }^{6} \mathrm{Li}\right)^{126} \mathrm{Sn}$ & 0000 & $0^{+}$ & 0.000 & 1.701 & 3.003 & 2.749 & 0.150 & 0.005 \\
\hline${ }^{122} \mathrm{Te}\left(\mathrm{d},{ }^{6} \mathrm{Li}\right)^{118} \mathrm{Sn}$ & 1758 & $0^{+}$ & 0.761 & 9.233 & 2.656 & 0.613 & 0.026 & 0.000 \\
\hline${ }^{124} \mathrm{Te}\left(\mathrm{d},{ }^{6} \mathrm{Li}\right){ }^{120} \mathrm{Sn}$ & 1875 & $0^{+}$ & 0.743 & 8.502 & 2.701 & 0.668 & 0.028 & 0.000 \\
\hline${ }^{126} \mathrm{Te}\left(\mathrm{d},{ }^{6} \mathrm{Li}\right){ }^{122} \mathrm{Sn}$ & 2090 & $0^{+}$ & 0.732 & 7.924 & 2.741 & 0.708 & 0.029 & 0.000 \\
\hline${ }^{128} \mathrm{Te}\left(\mathrm{d},{ }^{6} \mathrm{Li}\right)^{124} \mathrm{Sn}$ & $\left.2300^{\circ}\right)$ & $0^{+}$ & 0.697 & 7.183 & 2.712 & 0.736 & 0.030 & 0.000 \\
\hline${ }^{130} \mathrm{Te}\left(\mathrm{d},{ }^{6} \mathrm{Li}\right){ }^{126} \mathrm{Sn}$ & $\left.2600^{\circ}\right)$ & $0^{+}$ & 0.654 & 6.434 & 2.640 & 0.746 & 0.030 & 0.000 \\
\hline${ }^{112} \mathrm{Sn}\left(\mathrm{d},{ }^{6} \mathrm{Li}\right)^{108} \mathrm{Cd}$ & 0000 & $0^{+}$ & 0.890 & 9.673 & 2.209 & 0.395 & 0.022 & 0.000 \\
\hline${ }^{114} \mathrm{Sn}\left(\mathrm{d},{ }^{6} \mathrm{Li}\right){ }^{110} \mathrm{Cd}$ & 0000 & $0^{+}$ & 0.849 & 9.993 & 2.307 & 0.429 & 0.023 & 0.000 \\
\hline${ }^{116} \mathrm{Sn}\left(\mathrm{d},{ }^{6} \mathrm{Li}\right)^{112} \mathrm{Cd}$ & 0000 & $0^{+}$ & 0.803 & 10.156 & 2.445 & 0.482 & 0.024 & 0.000 \\
\hline${ }^{118} \mathrm{Sn}\left(\mathrm{d},{ }^{6} \mathrm{Li}\right)^{114} \mathrm{Cd}$ & 0000 & $0^{+}$ & 0.773 & 9.853 & 2.575 & 0.550 & 0.025 & 0.000 \\
\hline${ }^{120} \mathrm{Sn}\left(\mathrm{d},{ }^{6} \mathrm{Li}\right)^{116} \mathrm{Cd}$ & 0000 & $0^{+}$ & 0.764 & 9.269 & 2.668 & 0.616 & 0.027 & 0.000 \\
\hline${ }^{122} \mathrm{Sn}\left(\mathrm{d},{ }^{6} \mathrm{Li}\right)^{118} \mathrm{Cd}$ & 0000 & $0^{+}$ & 0.745 & 8.535 & 2.713 & 0.671 & 0.028 & 0.000 \\
\hline${ }^{124} \mathrm{Sn}\left(\mathrm{d},{ }^{6} \mathrm{Li}\right){ }^{120} \mathrm{Cd}$ & 0000 & $0^{+}$ & 0.735 & 7.953 & 2.752 & 0.711 & 0.029 & 0.000 \\
\hline${ }^{126} \mathrm{Sn}\left(\mathrm{d},{ }^{6} \mathrm{Li}\right){ }^{122} \mathrm{Cd}$ & 0000 & $0^{+}$ & 0.699 & 7.209 & 2.723 & 0.739 & 0.030 & 0.000 \\
\hline${ }^{128} \mathrm{Sn}\left(\mathrm{d},{ }^{6} \mathrm{Li}\right)^{124} \mathrm{Cd}$ & 0000 & $0^{+}$ & 0.656 & 6.457 & 2.651 & 0.749 & 0.030 & 0.000 \\
\hline${ }^{122} \mathrm{Te}\left(\mathrm{d},{ }^{6} \mathrm{Li}\right)^{118} \mathrm{Sn}$ & 2057 & $0^{+}$ & 0.000 & 1.576 & 2.462 & 0.338 & 0.003 & 0.000 \\
\hline
\end{tabular}

Amplitudes are calculated utilizing eq. (27) and the two-neutron and two-proton wave functions of appendix $C$.

") Estimated.

The procedures and approximations used in the calculations are described in subsect. 5.8 and the appendices $A$ to $D$. The two-neutron and two-proton spectroscopic amplitudes needed to calculate the $\alpha$-particle spectroscopic amplitude of eq. (27) were obtained from BCS and pairing wave functions. The calculated $\alpha$-particle spectroscopic amplitudes are listed in table 16 for the $J=0$ states and in table 17 for the $J \neq 0$ states. Zero-range DWBA calculations normalized absolutely to the $\alpha$-decay of ${ }^{148} \mathrm{Sm}$ were again used to calculate absolute cross sections. It should be noted that unlike two-nucleon transfer, the normalization permits prediction of absolute $\alpha$-transfer cross sections.

Noticing here only the rather good agreement between the experimental and calculated values, particularly for the Sn targets, a few general remarks about the spectroscopic and kinematic elements contained in the calculations will be discussed first.

The calculation of $\alpha$-spectroscopic amplitudes of eq. (27) for the given shell-model wave functions involve the summation over about 60 contributions for transitions to $J^{\pi}=0^{+}$states and 200 to 300 contributions for transitions to $J^{\pi} \neq 0^{+}$states. 
TABLE 17

Alpha-particle spectroscopic amplitudes $A_{1}^{Q L}$ for ${ }^{122} \mathrm{Te}\left(\mathrm{d},{ }^{6} \mathrm{Li}\right){ }^{11}{ }^{18} \mathrm{Sn}$ leading to $2^{+}$and $3^{-}$states as a function of the harmonic oscillator quantum number $Q$

\begin{tabular}{|c|c|c|c|c|c|c|c|c|c|}
\hline \multirow{2}{*}{ Reaction } & \multirow{2}{*}{$E_{\mathrm{x}}(\mathrm{keV})$} & \multirow{2}{*}{$J^{x}$} & \multirow{2}{*}{$L_{*}$} & \multirow{2}{*}{4} & \multicolumn{4}{|c|}{$100 A_{1}^{Q L}$} & \multirow[b]{2}{*}{$Q=22$} \\
\hline & & & & & $Q=14$ & $Q=16$ & $Q=18$ & $Q=20$ & \\
\hline \multirow[t]{4}{*}{${ }^{122} \mathrm{Te}\left(\mathrm{d},{ }^{6} \mathrm{Li}\right){ }^{118} \mathrm{Sn}$} & 1230 & $2^{+}$ & 0 & 2 & 0.923 & 4.979 & 0.524 & 0.003 & 0.000 \\
\hline & & & 2 & 0 & 0.985 & 3.306 & 1.087 & 0.088 & 0.002 \\
\hline & & & & & 1.908 & 8.285 & 1.611 & 0.091 & 0.002 \\
\hline & & & & & $Q=13$ & $Q=15$ & $Q=17$ & $Q=19$ & $Q=21$ \\
\hline \multirow[t]{3}{*}{${ }^{122} \mathrm{Te}\left(\mathrm{d},{ }^{6} \mathrm{Li}\right){ }^{118} \mathrm{Sn}$} & 2310 & $3^{-}$ & 0 & 3 & 0.000 & 2.668 & 1.736 & 0.059 & 0.000 \\
\hline & & & 3 & 0 & 2.752 & 13.649 & 5.303 & 0.415 & 0.020 \\
\hline & & & & & 2.752 & 16.317 & 7.039 & 0.474 & 0.020 \\
\hline
\end{tabular}

Amplitudes are calculated utilizing eq. (27) and the two-neutron and two-proton wave functions of appendix $C$.

The relative importance of each contribution is primarily determined by the last two terms in eq. (27) containing the two-nucleon spectroscopic amplitudes and structure amplitudes for the neutron and proton pairs. While the presence of spectroscopic strength is, of course, a necessary condition it is by no means sufficient. The structure factors which represent the overlap between the respective two-nucleon shell-model configuration in the target nucleus and in the $\alpha$-particle change enormously with quantum numbers. An inspection of structure factor tables ${ }^{69}$ ), for example, shows that low-spin contributions such as $\left(2 s_{\frac{1}{2}}^{2}\right)_{J_{\pi(v)}=0}$ up to $\left(1 \mathrm{~d}^{2}\right)_{J_{\pi(v)}=0}$ are strongly favored. In addition, certain high-spin contributions are also favored provided the two nucleons are in a "stretched" configuration with $J_{\pi(v)}=j_{>}+j_{<}$. For example, contributions with $\left(0 h_{\frac{11}{2}}, 1 h_{\frac{g}{z}}\right)_{J_{n(v)}=10^{+}}$are strongly favored, while

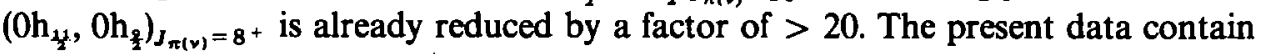
such a case where both spectroscopic and structure amplitudes are large. The heavy $\mathrm{Sn}$ and presumably also $\mathrm{Cd}$ nuclei have low-lying $7^{-}$states with a major $\left(\mathrm{Id}_{3}\right.$, $\left.0 \mathrm{~h}_{\frac{2}{2}}\right)_{J_{v}=7^{-}}$component which has a large structure amplitude.

Additional factors in eq. (27) also effect the magnitude of the $\alpha$-spectroscopic factor, but their dependence on quantum numbers is much weaker (see also Ichimura $e t$ $\left.\left.a l .{ }^{8}\right)\right)$. The factor $(B /(B-4))^{ \pm 2}$ which arises from recoil increases the cross sections with increasing harmonic oscilllator quantum number $Q(Q=14$ to $Q=22)$ by a factor of $\sim 1.3$. The reduced Wigner coefficient which reflects upon the coupling between neutron and proton pairs and the transferred $\alpha$-particle leads to a factor of $\sim 0.7$. (for $L_{\pi}=L_{v}=0$ ). The coefficient containing $Q$ ! leads to a factor of $\sim 0.55$ as an increase in $Q$ decreases the possibilities for combining four nucleons with the same c.m. motion and no internal excitation. The coefficient containing 4 ! has no systematic dependence on $Q$. Instead, it is a statistical factor which favors pickup of 

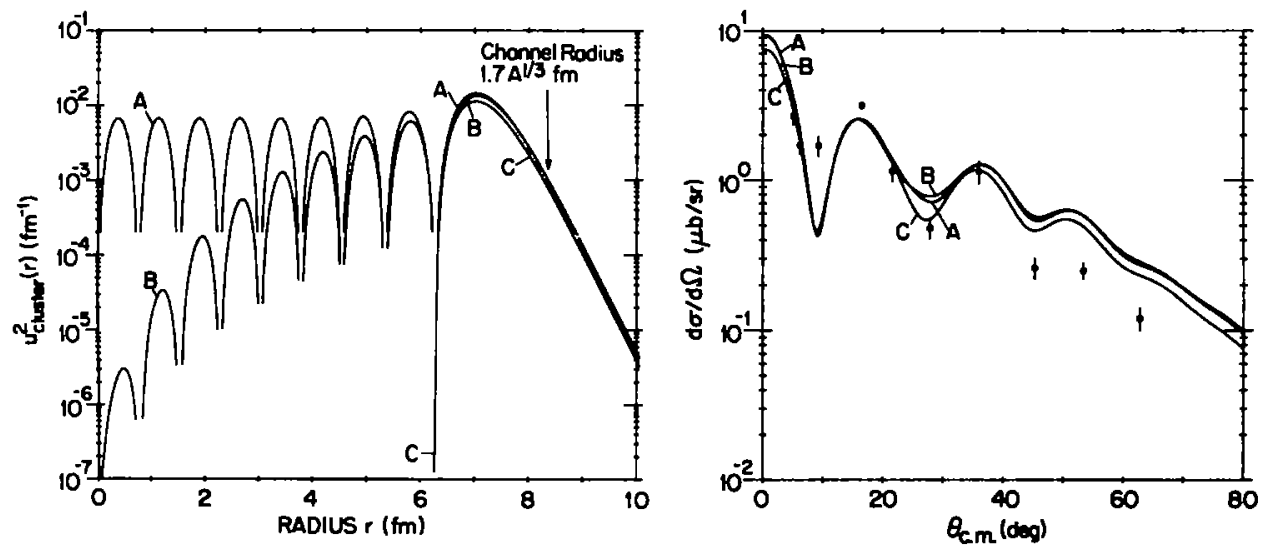

Fig. 10. $\alpha$-cluster wave functions and experimental and calculated angular distributions for ${ }^{122} \mathrm{Te}\left(\mathrm{d},{ }^{6} \mathrm{Li}\right)$ ${ }^{118} \mathrm{Sn}_{\mathrm{g} . \mathrm{f} .}$. The calculated angular distributions were obtained in zero-range DWBA with three $\alpha$-cluster wave functions (A) macroscopic, $u_{\text {clunter }}^{2}(r)=S_{\alpha} u_{\mathrm{DWBA}}^{2}(r)$; (B) microscopic, $u_{\text {clumer }}^{2}(r)=\varepsilon u_{\text {mieroecopic }}^{2}(r)$, see text; (C) macroscopic with lower radial cutoff at the outermost node, $u_{\text {cluuer }}^{2}(r)=0$ for $r<6.3 \mathrm{fm}$, $u_{\text {cluser }}^{2}(r)=0.78 S_{\alpha} u_{\mathrm{DWBA}}^{2}$ for $r>6.3 \mathrm{fm}$. The calculated angular distributions are averaged over $\Delta \theta= \pm 3^{\circ}$ to account for the experimental angular resolution. The values for $S_{a}$ and $\varepsilon$ are from tables 1 and 15.

nucleons from orbitals with different harmonic oscillator quantum numbers $q_{i}$. For proton and neutron pairs with different or identical quantum numbers $q_{i}$, for example, the cross section differs by a factor of six under otherwise similar conditions. The statistical factor is very important as it reduces the relative importance of those contributions which one often naively expects to dominate.

The calculation of the kinematic quantity $\beta_{\mathrm{L} M}^{Q}\left(k_{\mathrm{o}_{\mathrm{Li}}}, k_{\mathrm{d}}\right)$ of eq. (29) is greatly facilitated by the assumption that the form factors $f_{L M}^{Q}\left(r_{6 \mathrm{~L}}, r_{\mathrm{d}}\right)$ depend only on the harmonic oscillator quantum numbers $Q$ of the $\alpha$-cluster. This reduces the number of different form factors to 5 or 6 . The microscopic $\alpha$-cluster wave function for ${ }^{118} \mathrm{Sn}+\alpha$ is included in fig. 10. The figure displays the wave functions $u_{\text {cluater }}^{2}(r)$ with the resulting angular distributions for ${ }^{122} \mathrm{Te}\left(\mathrm{d},{ }^{6} \mathrm{Li}\right){ }^{118} \mathrm{Sn}$ for three assumptions. Case $\mathrm{A}$ is for the macroscopic $\alpha$-cluster wave functions $S_{\alpha} u_{Q=16}^{2}(r)$ where $S_{\alpha}$ of table 1 is the spectroscopic factor and $u_{Q=16}^{2}(r)$ is the normalized wave function with an assumed number of 8 radial nodes. Case B is for the microscopic $\alpha$-cluster wave function (table 17) $\varepsilon\left(\sum A^{Q 0} u_{Q}(r)\right)^{2}$. The enhancement factor $\varepsilon$ of table 15 which accounts for the ratio of experimental to calculated cross section has been included here. As can be seen, the cluster wave functions for case B is strongly suppressed in the nuclear interior while it is practically identical to that for case $A$ in the tail region including the chosen channel radius $s \approx 8.3 \mathrm{fm}$. The insensitivity to the shape of the wave function in the interior is of course taken as partial justification for the simplified procedure for calculating the microscopic form factor. Cross sections are not entirely independent of the interior region though, as can be seen from case $C$. Here, the macro- 
scopic cluster wave function A was used with an inner radial cutoff at the last radial node at $\sim 6.3 \mathrm{fm}$. The calculated cross section is increased by $Z 25 \%$, or alternately a slightly decreased cluster wave function suffices to generate the same cross section. It thus appears that contributions from the nuclear interior (but close to the surface) generate a small amount of destructive interference.

As the $\alpha$-cluster is picked up in the tail region of the $\alpha$-cluster wave function, the kinematic conditions of the reaction lead to another systematic dependence of the individual contributions on the harmonic oscillator quantum numbers $Q$. The wave functions $u_{Q}(r)$ increase at large radii with increasing $Q$ resulting in cross sections larger by a factor 1.5 to 2 for each additional node in the radial part of the $\alpha$-cluster wave function.

\subsection{TRANSITION TO STATES WITH $J^{*}=0^{+}$}

The experimental and calculated cross sections and experimental spectroscopic factors $S_{\alpha}$ for all Te(d, $\left.{ }^{6} \mathrm{Li}\right) \mathrm{Sn}$ and $\mathrm{Sn}\left(\mathrm{d},{ }^{6} \mathrm{Li}\right) \mathrm{Cd}$ ground state transitions and for transitions to certain excited $0^{+}$states are included in figs. 8, 9 and table 15. The cross sections are functions of the spectroscopic (table 16) and kinematic quantities while the phenomenologically defined $S_{\alpha}$ depend only on the former and are in principle independent of binding energies. However, it is the comparison with the absolute cross sections (normalized to $\alpha$-decay) of fig. 9 which provides the most insight.

The overall agreement between the experimental and calculated cross sections is excellent but certain systematic deviations also exist. The decrease in calculated cross section with neutron number $N$ is in part due to kinematic effects as the reaction $Q$-values become more negative. In addition, the $N$-dependence for all three types of states in fig. 9 is affected by the change in the fullness and emptiness of the various neutron orbitals. The maximum near $N=66$ occurs where contributions from $\left(1 \mathrm{~d}_{\frac{1}{q}}^{2}\right)_{L_{v}=0},\left(\mathrm{~s}_{\frac{1}{2}}^{2}\right)_{L_{v}=0}$, and $\left(1 \mathrm{~d}_{\frac{1}{2}}^{2}\right)_{L_{v}=0}$ are about equal. For $N<66,\left(1 \mathrm{~d}_{\frac{2}{2}}^{2}\right)_{L_{v}=0}$ dominantes; for $N>66,\left(1 d_{\frac{1}{2}}^{2}\right)_{L_{v}=0}$ dominantes. The calculated contributions from the proton pairs, mostly $\left(1 \mathrm{~d}_{\frac{1}{2}}^{2}\right)_{L_{\pi}=0}$, decrease with increasing $\mathrm{N}$ for the transitions to the' $\mathrm{Sn}$ ground states (the configuration $\left(\mathrm{gg}_{\frac{2}{2}}^{2}\right)_{L_{\pi}=0}$ is actually calculated to be stronger in the Te ground states but its transfer is inhibited by the structure amplitude); they are practically independent of $N$, mostly $\left(p_{\frac{z}{2}}^{-2}\right)_{L_{\pi}=0}$, for the transitions to the Sn pairing vibration states and the $\mathrm{Cd}$ ground states. Differences between the calculated cross sections for the transitions to the Sn ground states and the corresponding other two transitions is due to kinematic effects and the difference in proton orbitals, while that between the $\mathrm{Sn}$ pairing vibration states and the $\mathrm{Cd}$ ground states results from the dependence on reaction $Q$-values only (transition to pairing vibration states have more positive $Q$-values).

The agreement for the $\mathrm{Cd}$ g.s. transitions is excellent indicating that both neutron and proton orbitals are described correctly. The contributions result from the orbitals mentioned above with a harmonic oscillator quantum number $Q=14$ but other 
contributions are by no means negligible (see table 16), in particular contributions with $Q=16$.

Microscopic calculations for some of our data have been reported recently by Vitturi et al. ${ }^{13}$ ) using more detailed form factors. The results are similar to those reported here, particularly for the transitions to the Cd ground states.

Given the excellent agreement for the $\mathrm{Cd}$ ground states one might have expected equally good agreement for the $\$ n$ pairing vibration states as the reaction presumably involves the same neutron and proton orbitals. This is not the case. Instead, the experimental cross section for ${ }^{122} \mathrm{Te}\left(\mathrm{d},{ }^{6} \mathrm{Li}\right){ }^{118} \mathrm{Sn}$ (p.v.) is only about $75 \%$ of the calculated cross section, and when two or more neutron pairs are added the experimental cross section becomes vanishingly small. There exists a close correlation between this behavior and the excitation energies for these states which have a minimum at $N \approx 66$ (fig. 5). According to the interacting boson model ${ }^{21.24}$ ) the lowering of the excitation energies is due to increased collectivity which leads to static deformation in the middle of the neutron shell. This effect will also lead to a reduction in pairing strength. In the range $N=70$ to 78 and in the extreme rotational limit the calculated cross section should be reduced [eqs. (4) and (7) of ref. ${ }^{35}$ ) or eqs. (6.2) and (6.3) of ref. ${ }^{24}$ )] by factors of 0.25 to 0.40 . The experimental value of 0.75 for $N=70$ is quite reasonable considering the fact (see sect. 4) that the strength $\kappa$ of the boson quadrupole-quadrupole interaction is considerably below that for the rotational limit. However, the decrease in cross sections for the heavier targets is much stronger than calculated on the basis of the dependence on reaction $Q$-value, and must be due to another effect. While the calculated cross sections for the pairing vibration states in the Sn are overestimated, they are underestimated for the ground states. This is the behavior expected if mixing exists between ground and proton pairing vibration states. A similar situation ${ }^{5}$ ) seems to exist for ${ }^{64} \mathrm{Ni}$, which has been described by Broglia et al. ${ }^{12,13}$ ) by introducing an ad hoc mixing ratio of $25 \%$, and also in the $\mathrm{Zr}$ isotopes ${ }^{76}$ ).

The reaction ${ }^{126} \mathrm{Te}\left(\mathrm{d},{ }^{6} \mathrm{Li}\right){ }^{122} \mathrm{Sn}$ was chosen for a more detailed theoretical study. Expressing the ground and excited states as linear superpositions of the unperturbed ground and pairing vibration states (see fig. 11), the ensuing spectroscopic amplitudes can easily be calculated from the respective values of table 16 . The results for $\theta=16^{\circ}$ are displayed in fig. 11 together with the experimental cross sections for the ground and excited $0^{+}$states in ${ }^{126} \mathrm{Te}\left(\mathrm{d},{ }^{6} \mathrm{Li}\right)^{122} \mathrm{Sn}$. Small admixtures of a few percent have a pronounced effect on the calculated cross sections; admixtures of $30 \%$ change the cross sections by factors of $\sim 2$ and $\sim \frac{1}{10}$, respectively. A comparison between the experimental and calculated values suggests admixtures on the order of $20 \%$. Similarly, small admixtures of at most a few percent may be present in the Sn isotopes in the middle of the neutron shell while almost complete mixing seems to prevail for $N \geqq 76$. The origin of this mixing resulting in increased coherence for the protons in the ground states (in addition to the already existing neutron superfluidity) is unclear. It should be noted, though, that the interacting boson model ${ }^{21-24}$ ) predicts 


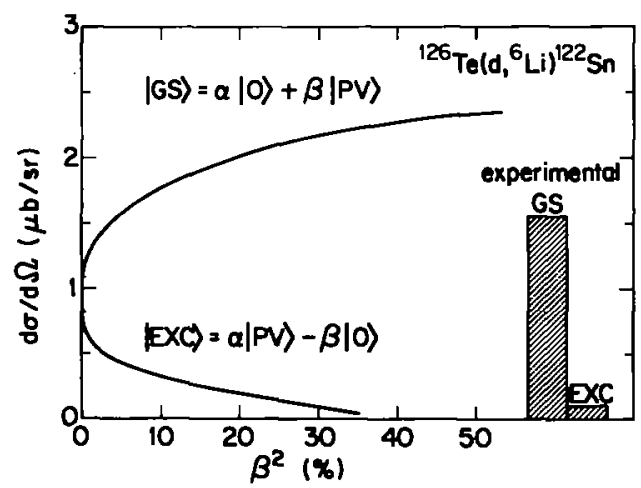

Fig. 11. Cross sections for ${ }^{126} \mathrm{Te}\left(\mathrm{d},{ }^{6} \mathrm{Li}\right){ }^{122} \mathrm{Sn}$ at $\theta=16^{\circ}$ for the $0^{+}$ground and pairing vibration states calculated with microscopic $\alpha$-cluster spectroscopic amplitudes and variable amounts of mixing between the two states. The calculated cross sections are normalized to the $\alpha$-decay ${ }^{148} \mathrm{Sm} \rightarrow{ }^{144} \mathrm{Nd}+\alpha$ (see text).

The respective experimental cross sections are represented by the bar diagram (compare to fig. 9).

increased overlap between the neutron components of the respective states for nuclei away from the middle of the neutron shell. This may be responsible despite the increased energy separation. Similar mixing of the proton pairing vibrational mode into the ground state of the $\mathrm{Ni}$ isotopes has been interpreted by Broglia et al. ${ }^{12}$ ) as the onset of a pairing phase transition towards proton superfluidity.

Some mixing with $0^{+}$states at higher excitation energies must also be present as transition strength to such states has been observed in the $\left({ }^{3} \mathrm{He}, \mathrm{n}\right)$ reaction $\left.{ }^{17}\right)$. These states are probably two-phonon excitations coupled to the proton pairing vibration (four-quasiparticle states). Such mixing is supported by the fact that only the high-spin members from $4^{+}$to $12^{+}$of the rotational bands ${ }^{42}$ ) based on the excited $0^{+}$states in ${ }^{112,114,116,118} \mathrm{Sn}$ are well described by a $J(J+1)$ dependence, with only $\sim 0.1 \%$ of $[J(J+1)]^{2}$, whereas the $2^{+}$and $0^{+}$members are depressed in energy by $\sim 100 \mathrm{keV}$ and $\sim 200 \mathrm{keV}$, respectively.

The experimentally observed decrease in cross section with neutron number for the Sn ground states is not fully reproduced by the calculations, and the disagreement is enhanced if mixing with the respective pairing vibration state is included. The reasons are unclear but may be due to proton core excitations in the ground states. The main component in the pickup from the Te ground states involves a $\left(1 \mathrm{~d}_{i}\right)^{2}$ neutron pair and a $\left(1 \mathrm{~d}_{\frac{5}{5}}\right)^{2}$ proton pair. Unlike the other types of transitions, these components have the same harmonic oscillator quantum numbers $Q_{v}=Q_{\pi}=8$ and are therefore not enhanced by the statistical factor in eq. (27). Therefore, pickup of $\left(0 \mathrm{~h}_{\frac{1}{2}}\right)^{2}$ and $\left(1 \mathrm{f}_{\frac{3}{3}}\right)^{2}$ neutron pairs with $Q_{v}=10$ which is practically independent of neutron numbers becomes important, and for ${ }^{130} \mathrm{Te}$ the calculated contributions with $Q=18$ even exceed those with $Q=16$. Proton pair excitations in the Sn ground states will shift the $Q_{\pi}=8$ component to $Q_{\pi}=6$ thus increasing the calculated cross section and leading to a stronger decrease with increasing neutron number. (The 
statistical factor gives an enhancement of $\sqrt{ } 6$ in the spectroscopic amplitude and the number of coherent contributions is increased.)

It appears that the effects observed in $\left(\mathrm{d},{ }^{6} \mathrm{Li}\right)$ on $\mathrm{Te}$ and $\mathrm{Sn}$ targets and attributed to mixing should be equally pronounced in two-proton pickup reactions as they seem to involve proton excitations only. The same should be true for the results in the $\mathrm{Ni}$ region $\left.{ }^{5}\right)$ and $\mathrm{Zr}$ region $\left.{ }^{76}\right)$. Indeed, preliminary results for $\left({ }^{6} \mathrm{Li},{ }^{8} \mathrm{~B}\right)$ seem to confirm this ${ }^{77}$ ).

The coherent mixing between ground and pairing vibration states ${ }^{12,13}$ ) has apparently so far only been observed for proton excitations and not for neutron excitations. One reasons could be the fact that proton pairing vibration states are generally energetically lower than neutron pairing vibration states and are therefore more susceptible to mixing.

Table 15 includes results for the $0^{+}$state at $2057 \mathrm{keV}$ in ${ }^{118} \mathrm{Sn}$. The calculated spectroscopic amplitudes are given in table 16. The calculations were performed on the assumption that the state is the lowest two-quasiparticle $0^{+}$state and both experimental and calculated cross sections are indeed quite small. It cannot be excluded though that the state is a coherent four-quasiparticle state (two-phonon vibration state).

The difference in size between the $\alpha$-cluster before and after the transfer results in a reduction in the calculated cross section which is not included in fig. 9 and table 15. The magnitude of the effect has been estimated in appendix $C$ as $0.6 \pm 0.25$ which implies enhancement factors $\varepsilon$ of about 1.5 to 2.0. It thus appears that even for the transitions to the $\mathrm{Cd}$ ground states slightly more pairing correlations, presumably for the protons, are required to account for the observed cross sections.

\subsection{TRANSITIONS TO STATES WITH $J^{\star} \neq 0^{+}$}

The spectroscopic factors for the states with $J_{\pi} \neq 0$ shown in fig. 8 display interesting regularities. Additional information comes from table 17 which gives the calculated $\alpha$-particle transfer amplitudes and table 15 which includes the experimental and calculated cross sections for the transitions to the $2^{+}$and $3^{-}$states in ${ }^{118} \mathrm{Sn}$ at $1230 \mathrm{keV}$ and $2310 \mathrm{keV}$, respectively.

The microscopic calculations were carried out on the assumption that for a given angular momentum transfer $L=J \neq 0$ with $L=L_{\pi}+L_{v}$ the superposition of only two coherent contributions, $\left(L_{\pi}, L_{v}\right)=(0, J)$ and $(J, 0)$, accounts for the observed cross section. A partial justification for this selection rule is included in appendix A. The $L_{\pi}=0$ and $L_{v}=0$ components in the transitions to both positive and negative parity states with $J>0$ in the $\mathrm{Sn}$ and Cd isotopes are presumably identical to those for the respective ground state transitions. This means that the components with $L_{v}=0$ are the same for both isotopes (mostly $\left(1 \mathrm{~d}_{\frac{4}{4}}^{2}+2 \mathrm{~s}_{\frac{1}{2}}^{2} 1 \mathrm{~d}_{\frac{1}{2}}^{2}\right)_{L_{v}=0}$ ) while those for $L_{\pi}=0$ are quite different (mostly $\left(1 d_{\frac{\xi}{2}}^{2}\right)_{L_{\pi}=0}$ for transitions to $\mathrm{Sn}$; mostly $\left(1 \mathrm{p}_{z}^{-2}\right)_{L_{\pi}=0}$ for transitions to $\mathrm{Cd}$ ). This is not expected to result in major differences in strength as can 
be seen from the g.s. transitions. The $L_{v} \neq 0$ components are also not expected to differ considerably for the two isotopes. Major differences, however, are expected for the $L_{\pi} \neq 0$ components as transitions to the $\mathrm{Sn}$ and $\mathrm{Cd}$ isotopes again involve proton pairs from different orbitals.

In the transitions to the even parity states in Sn the dominant components with $L_{\pi} \neq 0$ are calculated to come (neglecting core excitations) from proton pairs with $Z>50$ and $q_{i}=4$ (and $q_{i}=5$ ) while those for the states in Cd involve mostly proton pairs with $Z \leqq 50$ and $q_{i}=3$ as well as $q_{i}=4$ from the intruder $0 g_{q}$. Parity conservation requires $\Delta q_{i}=0$ or 2 for the two respective nucleons in the components with $L_{\pi} \neq 0$ and $L_{v} \neq 0$. Based on these comments and the microscopic analysis for the $2^{+}$state in ${ }^{118} \mathrm{Sn}$, the dominant contributions for the $2^{+}$states can be identified. The are given symbolically by

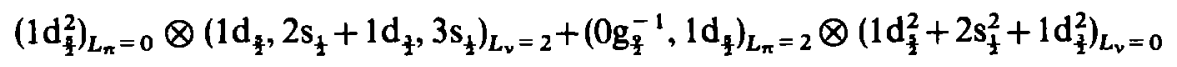

for the $\mathrm{Sn}$ isotopes and by

$$
\begin{aligned}
& \left(1 \mathrm{p}_{\frac{3}{1}}^{-2}\right)_{L_{\pi}=0} \otimes\left(1 \mathrm{~d}_{\frac{1}{3}}, 2 \mathrm{~s}_{\frac{1}{2}}+1 \mathrm{~d}_{\frac{3}{3}}, 2 \mathrm{~s}_{\frac{1}{2}}\right)_{L_{v}=2}+\left(1 \mathrm{p}_{\frac{1}{2}}^{-1}, 1 \mathrm{p}_{\frac{1}{2}}^{-1}+1 \mathrm{p}_{\frac{3}{2}}^{-2}\right)_{L_{\pi}=2} \\
& \otimes\left(1 \mathrm{~d}_{\frac{s}{2}}^{2}+2 \mathrm{~s}_{\frac{1}{2}}^{2}+1 \mathrm{~d}_{\frac{1}{2}}^{2}\right)_{L_{v}=0}
\end{aligned}
$$

for the $\mathrm{Cd}$ isotopes. The respective calculated amplitudes $(Q=16)$ for the transition to the $2^{+}$state in ${ }^{118} \mathrm{Sn}$ are included in table 17. Proton excitations are only moderately weaker than neutron excitations, and enhancement of the $\left(d,{ }^{6} \mathrm{Li}\right)$ over the $(\mathrm{p}, \mathrm{t})$ spectroscopic factors in fig. 8 is therefore expected. This is not observed, and it is concluded that the $\left(0 \mathrm{~g}_{\frac{2}{2}}^{-1}, 1 \mathrm{~d}_{\frac{1}{2}}\right)_{J=2}$ proton excitation in this state is probably weaker than predicted. This agrees also with the fact that the absolute cross section is slightly overestimated. Transitions to the $2^{+}$states in the Cd isotopes presumably include strong components with $\left(1 \mathrm{p}_{\frac{3}{2}}^{-1}, 1 \mathrm{p}_{\frac{f}{2}}^{-1}\right)_{J=2}$ and $\left(1 \mathrm{p}_{\frac{3}{2}}^{-2}\right)_{J=2}$. This should lead to a considerable enhancement of the $\left(d,{ }^{6} \mathrm{Li}\right)$ over the $(\mathrm{p}, \mathrm{t})$ spectroscopic strength which has indeed been observed (fig. 8).

The spectroscopic strength for the $4^{+}$states is greatly reduced due to the fact that the low- $j$ contributions which account for most of the $2^{+}$strength are not allowed. In the $L_{v}=4$ component (which is also responsible for the $(\mathrm{p}, \mathrm{t})$ strength), only weak contributions come from $\left(0 \mathrm{~g}_{\frac{2}{2}}, 1 \mathrm{~d}_{\frac{7}{4}}\right)_{L_{v}=4}$ (note that $\left.0 \mathrm{~g}_{\frac{7}{2}}, 1 \mathrm{~d}_{\frac{4}{4}}\right)_{L_{v}=2}$ contributes only 5 to $10 \%$ of $2^{+}$). Unlike the $2^{+}$strength, no enhanced $4^{+}$strength for the $\mathrm{Cd}$ isotopes is expected nor is it observed as the low- $j$ contributions are not possible. Small $L_{\pi}=4$ components may result from $\left(0 \mathrm{~g}_{\frac{3}{2}}^{-1}, 1 \mathrm{~d}_{\frac{1}{2}}\right)_{L_{\pi}=4}$ for the Sn isotopes and from $\left(0 \mathrm{~g}_{\frac{9}{2}}^{-2}\right)_{L_{\pi}=4}$ for the Cd isotopes.

Fig. 8 includes the strengths for $2_{2}^{+}$states in ${ }^{118,220} \mathrm{Sn}$ at $2043 \mathrm{keV}$ and $2098 \mathrm{keV}$, respectively. These states may carry proton quadrupole pairing vibration strength, and they have also been identified ${ }^{42}$ ) as members of rotational bands based on the $0_{2}^{+}$states at $1758 \mathrm{keV}$ and $1975 \mathrm{keV}$, respectively. The observed strengths are compatible with such assignments. 
The discussion of the transitions to the odd-parity states in the $\mathrm{Sn}$ and $\mathrm{Cd}$ isotopes employs essentially the same arguments as before. A transition to a $3^{-}$state, for example, will again contain a coherent superposition of contributions $\left(L_{*}, L_{v}\right)=(0,3)$ and $(3,0)$ whereby the $L_{\pi}=0$ and $L_{v}=0$ components are those of the respective ground state transitions (the $L_{\pi}=0$ component differs for transitions to $\mathrm{Sn}$ and $\mathrm{Cd}$ ). The relative $L_{v}=3$ components are not likely to differ much for the two isotopes while those with $L_{\pi}=3$ may differ considerably. Unlike transitions to even-parity states, however, parity conservation requires $\Delta q_{i}=1$ for the two respective nucleons in the components with $L_{\pi} \neq 0$ and $L_{v} \neq 0$. Therefore, core excitations and the intruder orbit $\mathrm{Oh}_{\frac{11}{2}}$ are expected to play a more important role.

The transition to the $3^{-}$state in ${ }^{118} \mathrm{Sn}$ which has been calculated microscopically (see tables 15 and 17$)$ has only a weak $L_{v} \neq 0$ component, $\left(1 \mathrm{~d}_{\hat{q}}, 0 \mathrm{~h}_{u_{2}}\right)_{L_{v}=3}$, involving the $0 h_{12}$ intruder level. This explains the relatively weak $(p, t)$ strength. However, core excitation with $L_{\pi}=3$ from $\left(1 \mathrm{p}_{\frac{1}{2}}^{-1}, 1 \mathrm{~d}_{\frac{1}{4}}\right)_{L_{\pi}=3},\left(1 \mathrm{p}_{\frac{3}{3}}^{-1}, 1 \mathrm{~d}_{\frac{1}{2}}\right)_{L_{\pi}=3},\left(1 \mathrm{p}_{\frac{1}{2}}^{-1}, 1 \mathrm{~d}_{\frac{1}{3}}\right)_{L_{\pi}=3}$ and $\left(1 p_{\frac{1}{3}}^{-1}, 0 g_{\frac{z}{3}}\right)_{L_{\pi}=3}$ contributes rather strongly explaining the enhanced $\alpha$-transfer. These transitions should not change strongly with neutron number, and indeed no strong variation in strength is seen. The components with $L_{\pi}=3$ are not possible for the transitions to the $3^{-}$states in Cd unless proton core excitations in the Sn ground states are introduced. However, the $\mathrm{Og}_{\frac{q}{3}}$ orbit should play an important role. The components $\left(0 \mathrm{~g}_{\frac{q}{3}}^{-1}, 0 \mathrm{f}_{\frac{z}{2}}^{-1}\right)_{L_{\pi}=3},\left(0 \mathrm{~g}_{\frac{q}{2}}^{-1}, 1 \mathrm{p}_{\frac{1}{2}}^{-1}\right)_{L_{\pi}=0}$ and $\left(0 \mathrm{~g}_{\frac{q}{2}}^{-1}, 1 \mathrm{p}_{\frac{3}{3}}^{-1}\right)_{L_{\pi}=0}$ seem to account for the even stronger transitions to the $3^{-}$states in Cd. It is not clear though why the strength increases with neutron number.

The number of contributions added coherently to calculate the microscopic cross section to the $3^{-}$state in ${ }^{118} \mathrm{Sn}$ is very large, but many are quite small. Nevertheless, there are about 4 rather strong contributions which involve neutron excitations and about 24 which involve proton excitations. As pointed out by Kurath and Towner ${ }^{9}$ ) it is this rather large number of components in the wave function which is responsible for both strong $\alpha$-transfer and strong inelastic scattering.

The relatively strong transitions to the $5^{-}$states in $\mathrm{Sn}$ are not completely understood. Apparently neutron and proton excitations play a role, and the components

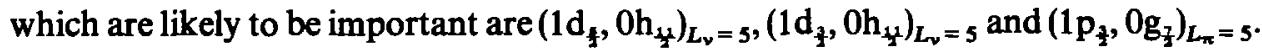
However, none of these are expected to be large nor favored by structure factors.

The transitions to the $7^{-}$states in Sn display a dramatic increase in $\alpha$-spectroscopic strength with increasing neutron number. This favored $\alpha$-transfer is due to a combination of circumstances including the fact that the $7^{-}$states in the heavier $\mathrm{Sn}$ isotopes have an almost pure $\left.{ }^{19}\right)$ neutron configuration of $\left(0 \mathrm{~h}_{4}, 1 \mathrm{~d}_{\frac{1}{2}}\right)_{J=7}$. This is also related to the low excitation energy (see fig. 5) and results in strong two-nucleon spectroscopic amplitudes according to eq. (45) when combined with the fullness parameters of $v\left(1 \mathrm{~d}_{3}\right) \approx 0.9$ and $v\left(0 \mathrm{~h}_{43}\right) \approx 0.8$ for the heavier $\mathrm{Sn}$ isotopes. Finally, the structure amplitude for the above configuration is also large as it is a favored "stretched" configuration with $J=j_{>}+j_{<}$. Contributions from proton excitations are presumably very small as they would require core excitations of the type $\left(0_{\frac{7}{7}}, 0 f_{\frac{7}{3}}^{-1}\right)_{J=7}$. 
It is therefore concluded that the observed $\alpha$-spectroscopic strength results almost entirely from

$$
\left(1 d_{\frac{b}{2}}^{2}\right)_{L_{\pi}=0} \otimes\left(0 h_{\frac{11}{2}}, 1 d_{\frac{3}{3}}\right)_{L_{v}=7}
$$

States with unknown spin-parity assignments at $1269 / 1286 \mathrm{keV}$ in ${ }^{118} \mathrm{Cd}$ and at $1323 \mathrm{keV}$ in ${ }^{120} \mathrm{Cd}$ are strongly excited. These states are likely candidates for $J^{\pi}=7^{-}$ since strong transitions to such states are expected. The excitation energies should indeed be lower than for the respective states in the $\mathrm{Sn}$ isotopes since $\mathrm{Sn}$ is semimagic (see also fig. 5). Neutron excitations from $\left(0 \mathrm{~h}_{\frac{1}{2}}, 1 \mathrm{~d}_{\frac{3}{2}}\right)_{J=7}$ should again be strong, but unlike transitions to the $7^{-}$states in $\mathrm{Sn}$, coherent proton excitations may also contribute significantly possibly accounting for the unusually large cross sections. The observed cross section may thus result from

$$
\left(1 \mathrm{p}_{\frac{3}{2}}^{-2}\right)_{L_{\pi}=0} \otimes\left(0 \mathrm{~h}_{\frac{14}{2}}, 1 \mathrm{~d}_{\frac{1}{3}}\right)_{L_{v}=7}+\left(0 \mathrm{~g}_{\frac{3}{2}}^{-1}, 0 \mathrm{f}_{\frac{3}{2}}^{-1}\right)_{L_{\pi}=7} \otimes\left(1 \mathrm{~d}_{\frac{3}{2}}^{2}\right)_{L_{v}=0} .
$$

Considering two-nucleon spectroscopic and structure amplitudes together with the sequence of shell-model orbits, it appears that transitions to certain high-spin states with odd parity should be favored in $\alpha$-transfer below magic numbers, e.g. $5^{-}$and $7^{-}$below, $Z, N=50$ or $7^{-}$and $9^{-}$below $Z, N=82$.

\section{Summary}

Alpha-cluster pickup via (d, $\left.{ }^{6} \mathrm{Li}\right)$ on most even- $A$ Te and $\mathrm{Sn}$ targets has been studied at $E_{\mathrm{d}}=33 \mathrm{MeV}$. Spectra and angular distributions have been measured. Excitation energies of new states and the mass excess of ${ }^{120} \mathrm{Cd}$ have been determined and spin-parity assignments have been made. DWBA theory has been used to extract $\alpha$-cluster spectroscopic factors and reduced widths from the data. Absolute reduced widths deduced from finite range DWBA analysis of $\alpha$-pickup and from $\alpha$-decay $\left({ }^{148} \mathrm{Sm} \dot{\rightarrow}{ }^{144} \mathrm{Nd}+\alpha\right)$ disagree by a factor of $\sim 16$. The spectroscopic factors for $\left(\mathrm{d},{ }^{6} \mathrm{Li}\right)$ and $(\mathrm{p}, \mathrm{t})$ scale very closely for the ground state transitions but $\alpha$-pickup is enhanced for transitions to most excited states due to coherent contributions from the excitation of proton pairs and neutron pairs. A semi-microscopic analysis has been performed using the formulation of Kurath and Towner ${ }^{9}$ ) with neutron BCS and proton pairing wave functions ${ }^{13,18,20}$ ). The experimental and calculated cross sections for the transitions to the $0^{+}$ground states in $\mathrm{Sn}$ and $\mathrm{Cd}$ agree well but the former seem to require added proton core excitations. The $0^{+}$proton pairing vibrational state in ${ }^{118} \mathrm{Sn}$ is populated with a strength about equal to that seen ${ }^{17}$ ) in $\left({ }^{3} \mathrm{He}, \mathrm{n}\right)$. The strength disappears rapidly with increasing neutron excess. This effect can be explained by introducing mixing between the $0^{+}$ground and pairing vibrational scates ${ }^{12,13}$ ). The excitation energies for the proton pairing vibrational states in Sn exhibit a pronounced minimum in the middle of the neutron shell as a result of increased collectivity ${ }^{42,43}$ ) predicted by the interacting boson approximation (IBA) [refs. ${ }^{21-24)}$ ]. 
We thank the operating and scientific staff of the Brookhaven National Laboratory Tandem Van de Graaff Laboratory for their assistance. We acknowledge the support of $\mathrm{H}$. Song and $\mathrm{D}$. Overway at various stages of the experiment. Stimulating and helpful discussions with K. T. Hecht and F. Iachello are highly appreciated.

\section{Appendix A}

ALPHA-PARTICLE SPECTROSCOPIC AMPLITUDES FROM SHELL-MODEL WAVE FUNCTIONS

Alpha-particle spectroscopic amplitudes can be expressed as a coherent sum of two-proton and two-neutron spectroscopic amplitudes. The equations given below for the pickup reaction $\mathrm{B}\left(\mathrm{d},{ }^{6} \mathrm{Li}\right) \mathrm{A}$ are based on those of Kurath and Towner ${ }^{9}$ ) but $\mathrm{SU}_{3}$ group theory notation is used and several simplifications are introduced. The relevant angular momentum couplings for ${ }^{6} \mathrm{Li}=\mathrm{d}+\alpha$ and $\mathrm{B}=\mathrm{A}+\alpha$ are displayed in fig. 12.

(i) The cluster representation of ${ }^{6} \mathrm{Li}$ is assumed to be based on

$$
j_{\sigma_{\mathrm{Li}}}=j_{\mathrm{d}}+J_{\alpha}=j_{\mathrm{d}}+j \alpha+L
$$

with $j_{\sigma_{\mathrm{Li}}}=j_{\mathrm{d}}=1$ and $j_{\alpha}=L=J_{\alpha}=0$. Thus, the intrinsic spin of the $\alpha$-cluster is taken as $j_{\alpha}=0$ like the $\alpha$-particle ground state and the relative motion between $\alpha$-cluster and d-cluster is approximated by a pure s-state with no d-state admixtures. The harmonic oscillator quantum number $\bar{Q}=2 N+L$ for the relative motion is fixed at $\bar{Q}=2$ (or $\bar{Q}=0$ ) with $L=0$ and one (or zero) radial node outside $r=0$.
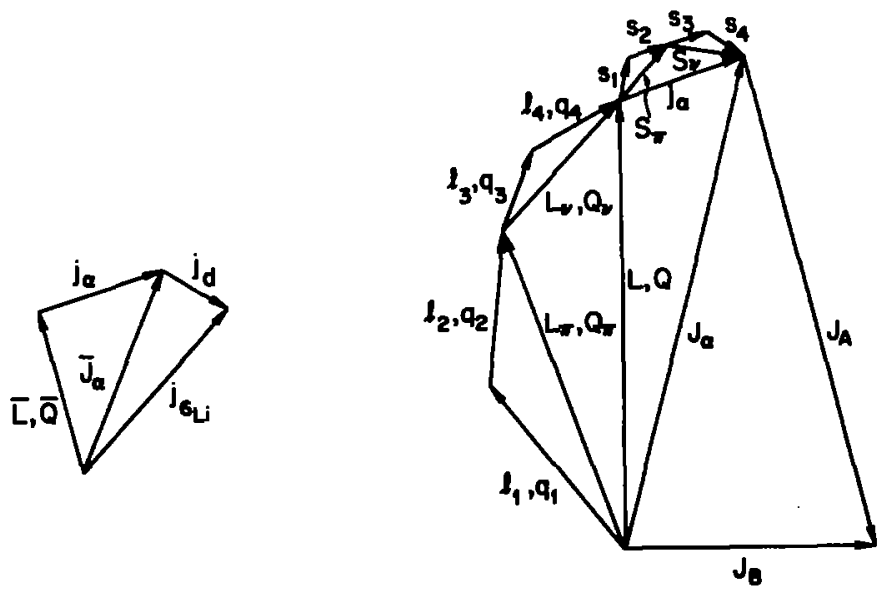

Fig. 12. Angular momentum couplings for the light $\left({ }^{6} \mathrm{Li}=\mathrm{d}+\alpha\right)$ and heavy $(B=A+\alpha)$ particle in the pickup reaction $\mathrm{B}\left(\mathrm{d},{ }^{6} \mathrm{Li}\right) \mathrm{A}$. Orbital angular momenta are indicated together with the respective harmonic oscillator quantum numbers ( $L$ with $Q=2 N+L$, for example). 
(ii) The cluster representation of the heavy nucleus $B$ is based on

$$
J_{\mathrm{B}}=\boldsymbol{J}_{\mathrm{A}}+\boldsymbol{J}_{\alpha}=\boldsymbol{J}_{\mathrm{A}}+\boldsymbol{J}_{\alpha}+\boldsymbol{L}
$$

Cross sections are written for transitions between even-even nuclei, thus $J_{B}=0$ (or $J_{\mathrm{A}}=\mathbf{0}$ for stripping). Furthermore, both neutrons and protons in the target ground state are assumed to be independently coupled to spins of zero, $J_{p}=J_{n}=0$ (or $J_{\mathrm{p}}=J_{\mathrm{n}}=0$ ). With $j_{\alpha}=0$, the angular momentum transfer in the reaction is

$$
L=J_{\alpha}=J_{\mathrm{A}} \text { (or } J_{\mathrm{B}} \text { for stripping), }
$$

with the harmonic oscillator quantum number $Q=2 N+L$ (see subsect. 5.8). Furthermore, since the transfer involves a coherent superposition of proton and neutron pairs in singlet-even states $(S=0, T=1)$ we have

$$
\begin{aligned}
& S_{\pi}=s_{1}+s_{2}=0, \\
& S_{v}=s_{3}+s_{4}=0,
\end{aligned}
$$

and therefore

$$
\boldsymbol{L}=\boldsymbol{L}_{\boldsymbol{\pi}}+\boldsymbol{L}_{\boldsymbol{v}}
$$

with

$$
\begin{aligned}
& L_{\pi}=j_{1}+j_{2}=l_{1}+l_{2}, \\
& L_{v}=j_{3}+j_{4}=l_{3}+l_{4},
\end{aligned}
$$

where $j_{i}, l_{i}$ and $s_{i}$ refer to the individual nucleons.

(iii) The spectroscopic quantity $\beta_{L M}^{Q}$ of eqs. (24) and (29) is assumed to depend only on the angular momentum transfer $L$ with projection $M$ (see fig. 12) and the total number of harmonic oscillator quanta

$$
Q=Q_{\pi}+Q_{v}
$$

with

$$
\begin{aligned}
& Q_{\pi}=q_{1}+q_{2}, \\
& Q_{v}=q_{3}+q_{4} .
\end{aligned}
$$

The quantity $\beta_{q_{M}}$ is assumed to be independent of the quantum numbers of the individual nucleons.

(iv) The size of the $\alpha$-cluster is assumed to be the same before and after the transfer. However, an estimate of $\alpha$-cluster size effects will be presented in appendix C. 
The differential cross section for $\left(\mathrm{d},{ }^{6} \mathrm{Li}\right) \alpha$-cluster pickup reactions can be written as ${ }^{9}$ )

$$
\frac{\mathrm{d} \sigma}{\mathrm{d} \Omega}\left(\mathrm{d},{ }^{6} \mathrm{Li}\right)=\frac{\mu_{6 \mathrm{Li}} \mu_{\mathrm{d}}}{\left(2 \pi \hbar^{2}\right)^{2}} \frac{k_{6 \mathrm{Li}}}{k_{\mathrm{d}}} \frac{1}{3} \sum_{M}\left|\sum_{Q} B_{L}^{Q} \beta_{L M}^{Q}\right|^{2},
$$

where $B_{L}^{Q}$ and $\beta_{L M}^{Q}$ are factors describing the dependence on the spectroscopic and kinematic elements of the reaction. The spectroscopic quantity $B_{L}^{Q}$ can be factorized,

$$
B_{L}^{Q}=i^{L} \sqrt{3} A_{1}^{Q L^{*}} A_{2}^{\bar{Q} \bar{L}}
$$

where $A_{1}$ and $A_{2}$ are the $\alpha$-particle spectroscopic amplitudes for $\mathrm{B}=\mathrm{A}+\alpha$ and ${ }^{6} \mathrm{Li}=\mathrm{d}+\alpha$, respectively. The amplitudes for the heavy particles are given by ${ }^{8,9}$ )

$$
A_{1}^{Q L}=\left(\frac{B}{B-4}\right)^{\frac{1 Q}{L}} \sum_{\Gamma}\left\langle\psi(B) \| \chi^{\Gamma \dagger}|| \psi(A\rangle\left\langle\phi^{\alpha} \phi_{L M}^{Q}\left(r_{a A}\right) \mid \psi_{\text {shell model }}^{\Gamma}\right\rangle\right.
$$

The factor in front of the summation arrives from recoil, and $B$ is the target mass (in pickup). The double-barred matrix element is a coefficient of fractional parentage, and the second factor in the summation, the four-nucleon structure amplitude, is the overlap of the $\alpha$-cluster wave function (internal and relative motion) with the four-nucleon shell-model wave function specified by quantum numbers $\Gamma$. The phenomenologically defined $\alpha$-particle spectroscopic factor is given by $S_{\alpha}=\left|A_{1}^{Q L}\right|^{2}$ provided the spectroscopic amplitude for a particular value of $Q$ dominates.

Introducing the expressions of Kurath and Towner ${ }^{9}$ ) together with the simplifications mentioned above, the $\alpha$-particle spectroscopic amplitudes of eq. (26) can be expressed in terms of two-proton and two-neutron parentage amplitudes as

$$
\begin{aligned}
A_{1}^{Q L} & =\left(\frac{B}{B-4}\right)^{ \pm Q} \sum_{\substack{L_{\pi}+L_{v}=L \\
Q_{\pi}+Q_{v}=Q}} \sum_{j_{1} \geq j_{2}} \sum_{J_{3} \geq j_{4}}\left(\frac{2 L+1}{\left(2 L_{\pi}+1\right)\left(2 L_{v}+1\right)}\right)^{\frac{1}{t}} \\
& \times\left\langle\left(Q_{\pi} 0\right) L_{\pi}\left(Q_{v} 0\right) L_{v} \|(Q 0) L\right\rangle\left(\frac{Q !}{4^{Q} q_{1} ! q_{2} ! q_{3} ! q_{4} !}\right)^{\frac{1}{2}}\left(\frac{4 !}{a ! b ! c ! d !}\right)^{\frac{1}{t}} \\
& \times G\left(l_{1} j_{1} l_{2} j_{2} L_{\pi} q_{1} q_{2} Q_{\pi}\right)\left\langle\psi^{L_{\pi}=0}\left\|\chi^{L_{n}\left(j_{1} j_{2}\right) \dagger}\right\| \psi^{L_{\pi}}\right\rangle \\
& \times G\left(l_{3} j_{3} l_{4} j_{4} L_{v} q_{3} q_{4} Q_{v}\right)\left\langle\psi^{\bar{L}_{v}=0}\left\|\chi^{L_{v}\left(U_{3} j_{4}\right) \dagger}\right\| \psi^{L_{v}}\right\rangle .
\end{aligned}
$$

Eq. (27) makes use of two-nucleon structure amplitudes and angular momentum coupling coefficients in the SU(3) coupling scheme. The last two terms in eq. (27) for the proton and neutron pairs, $G()\langle\|\|\rangle$, represent the well known products of two-nucleon structure amplitude times two-nucleon parentage (or spectroscopic) amplitude. The structure amplitudes are general functions of the various quantum numbers while the spectroscopic amplitudes depend on the individual pair of nuclei. The quantity $\left\langle\left(Q_{\pi} 0\right) L_{\pi}\left(Q_{v} 0\right) L_{v} \|(Q 0) L\right\rangle$ is a $S U(3) \mid R(3)$ reduced Wigner coefficient and it describes the angular momentum coupling $L_{n}+L_{v}=L$ with harmonic 
oscillator quanta $Q_{\pi}+Q_{\nu}=Q$ for neutron and proton pairs and the transferred $\alpha$-particle. The factors containing $Q$ ! and 4 ! account for the number of possible combinations of quantum numbers $q_{l}$ to form $Q$ and of nucleons distributed over the quantum numbers $q_{i}$ to form an entity of four nucleons, the $\alpha$-cluster, respectively. Here, $a+b+c+d=4$, and each of the four quantities gives the number of nucleons with equal harmonic oscillator quantum numbers $q_{i}$ (for example $a=3, b=1$, $c=d=0$ for $\left.q_{1}=q_{2}=q_{3} \neq q_{4}\right)$. The two-nucleon structure amplitudes $G\left(l_{1} j_{1} l_{2} j_{2} L_{\pi} q_{1} q_{2} Q_{\pi}\right)$ and $G\left(l_{3} j_{3} l_{4} j_{4} L_{v} q_{3} q_{4} Q_{v}\right)$ of eq. (27) are intimately related but not identical to the structure amplitudes $G_{N L S J}^{l_{1} j_{1} l_{2} j_{2}}$ and $G_{N L S J}^{l_{3} j_{3} l_{4} j_{4}}$ defined by Glendenning $\left[\right.$ refs. $\left.\left.{ }^{64,66.69}\right)\right]$. Instead (except for phase factors),

$$
G\left(l_{1} j_{1} l_{2} j_{2} L_{\pi} q_{1} q_{2} Q_{\pi}\right)=\left(\frac{q_{1} ! q_{2} ! 2^{Q_{\pi}}}{Q_{\pi} !}\right)^{\frac{1}{t}}\left(\frac{\tilde{a} ! \tilde{b} !}{2 !}\right)^{\frac{1}{2}} G_{N L L}^{l_{1} j_{1} l_{2} j_{2}},
$$

and accordingly for $G\left(l_{3} j_{3} l_{4} j_{4} L_{v} q_{3} q_{4} Q_{v}\right)$. As before we have $(\tilde{a}, \tilde{b})=(2,0)$ for $q_{i}=q_{2}$ and $(\tilde{a}, \tilde{b})=(1,1)$ for $q_{1} \neq q_{2}$. The difference in the two spectroscopic amplitudes results from different normalization. The structure factors of eq. (27) are normalized to unity while those of Glendenning ${ }^{64,69}$ ) and Kurath and Towner ${ }^{9}$ ) are normalized to the SU(3) limit. The structure amplitudes of eq. (28) assume equal size of the twonucleon cluster before and after the transfer. The effect of a difference in size will be discussed in appendix $\mathbf{C}$.

The $\alpha$-particle spectroscopic amplitudes $A_{1}^{Q L}$ of eq. (27) were calculated on the assumption $\left(L_{x}, L_{v}\right)=(0,0)$ for transitions to final states with $J^{\pi}=0^{+}$, and on the assumption of a coherent superposition of the contributions with $\left(L_{\pi}, L_{v}\right)=(0, J)$ and $(J, 0)$ for transitions to final states with $J^{\pi} \neq 0^{+}$(for each value of $Q$ ). Table 18 presents a partial justification for this (weak) selection rule. The table lists the quantity $(2 L+1)^{\frac{1}{2}}\left(2 L_{\pi}+1\right)^{-\frac{1}{2}}\left(2 L_{v}+1\right)^{-\frac{1}{1}}\left\langle\left(Q_{\pi} 0\right) L_{\pi}\left(Q_{v} 0\right) L_{v} \|(Q 0) L\right\rangle$ as a function of $L_{\pi}$ and $L_{v}$ for fixed values of $L, Q_{\pi}$ and $Q_{v}$. It shows that angular momentum coupling in

TABLE 18

Dependence of the quantity $(2 L+1)^{1 / 2}\left(2 L_{\pi}+1\right)^{-1 / 2}\left(2 L_{v}+1\right)^{-1 / 2}\left\langle\left(Q_{\pi} 0\right) L_{\pi}\left(Q_{v} 0\right) L_{v} \|(Q 0) L\right\rangle$ on $L_{\pi}$ and $L_{v}$ for $L=0,2$ and 3 and $Q_{\pi}=8$ or $7, Q_{v}=8$ or $7\left(L_{\pi}+L_{v}=L_{;} Q_{\pi}+Q_{v}=Q\right)$
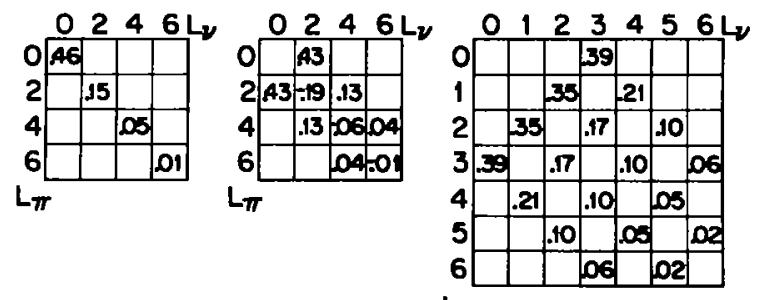

$\begin{array}{lll}O^{+} \rightarrow 0^{+} & O^{+} \rightarrow 2^{+} & O^{+} \rightarrow 3^{-} \\ Q_{\pi}=8 & Q_{\pi}=8 & Q_{\pi}=8(7) \\ Q_{\nu}=8 & Q_{\nu}=8 & Q_{\nu}=7(8)\end{array}$


a $0^{+} \rightarrow 0^{+}$transitions favors $\left(L_{\pi}, L_{v}\right)=(0,0)$ over $\left(L_{\pi}, L_{v}\right)=(2,2)$ by a factor of almost 10 in the squares of the amplitudes. This factor is over and beyond the dependence on the two-proton and two-neutron spectroscopic and structure amplitudes which usually strongly favor $L_{x}=0$ and/or $L_{v}=0$. Similarly, in a $0^{+} \rightarrow 2^{+}$transition $\left(L_{\pi}, L_{v}\right)=(0,2)$ and $(2,0)$ are favored over $\left(L_{\pi}, L_{v}\right)=(2,2)$ by a factor of 5 . In addition, the two-nucleon spectroscopic and structure amplitudes are likely to again favor the transition where one of the pairs is transferred with $L_{\pi(v)}=0$. In transitions to states with $J>2$ angular momentum coupling between the pairs will not favor the transfer with $L_{\pi}=0$ or $L_{v}=0$. If $J^{\pi}=7^{-}$, for example, the transfer with $\left(J_{\pi}, J_{v}\right)=(0,7)$ is actually disfavored by a factor of $\sim 3$ with regard to $\left(J_{\pi}, J_{v}\right)=(4,3)$. However, even here the spectroscopic and structure amplitudes are likely to favor the transfer with $L_{\pi}=0$ or $L_{v}=0$, but it is quite conceivable that the assumed selection rule may not hold for transitions to certain high-spin states.

\section{Appendix B}

FORM FACTORS AND KINEMATIC DEPENDENCE

The kinematic dependence of the differential cross section of eq. (24) is given by

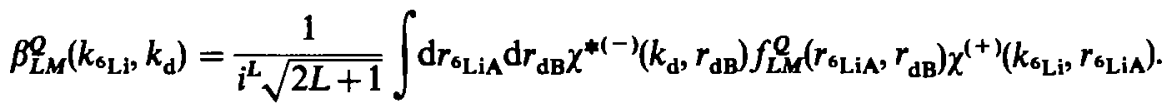

Here, $\chi_{\mathrm{d}}$ and $\chi_{\sigma_{\mathrm{Li}}}$ are the distorted wave generated by the deuteron and ${ }^{6} \mathrm{Li}$ optical potentials. The quantity $f_{L M}^{Q}$ is the form factor in the post representation,

$$
f_{L M}^{Q}\left(r_{\mathrm{L} L \mathrm{~A}}, r_{\mathrm{dB}}\right)=\phi_{L M}^{Q}\left(r_{\alpha \mathrm{A}}\right) V_{\mathrm{d} \alpha}\left(r_{\mathrm{d} \alpha}\right) \phi_{\bar{L} \bar{M}}^{\bar{Q}}\left(r_{\mathrm{d} \alpha}\right) \text {. }
$$

The well known zero-range approximation can be introduced if desired by setting

$$
V_{\mathrm{d} \alpha}\left(r_{\mathrm{d} \alpha}\right) \phi_{\bar{L} \bar{M}}^{\bar{Q}}\left(r_{\mathrm{d} \alpha}\right)=D_{0} \delta\left(r_{\mathrm{d} \alpha}\right)
$$

but requires the use of a normalization constant $D_{0}$ which can in principle be calculated. The dimensionless normalization factor $\mathscr{N}$ is obtained from $\mathcal{N}=\left(D_{0} / 10^{2}\right.$ $\left.\mathrm{MeV} \cdot \mathrm{fm}^{\frac{1}{3}}\right)^{2}$. The functions $\phi_{\bar{L} \bar{M}}^{\bar{Q}}\left(r_{\mathrm{da}}\right)$ and $\phi_{\overline{L M}}^{Q}\left(r_{\alpha \mathrm{A}}\right)$ are the bound state wave functions for the $\alpha$-cluster before and after the transfer. The wave functions $\phi_{\mathrm{LM}}^{Q}$ are generated for each required harmonic oscillator quantum number $Q$ in Woods-Saxon potential wells by adjusting the well depths to fit the $\alpha$-particle binding energy.

\section{Appendix C}

\section{ALPHA-PARTICLE SIZE EFFECTS}

The size of the transferred $\alpha$-cluster is different before and after the reaction. This effect will reduce the calculated cross sections. First- and second-order correction terms for the case where neutron and proton pairs occupy the same oscillator orbit 
have been reported by Hecht ${ }^{78}$ ). These expressions will be applied to obtain an estimate for the magnitude of the effect.

The change in size of the $\alpha$-cluster will affect the four-nucleon structure amplitude $G$ defined by the second term in the sum of eq. (26). If the internal wave function for the $\alpha$-particle is expanded in terms of harmonic oscillator wave functions for the parent (or core) nucleus, the amplitudes $G$ can be expressed in terms of the overlap integral $\Omega_{n 0}=\left\langle\phi_{000}^{\alpha}\left(v_{\sigma_{\mathrm{Li}}}\right) \mid \phi_{n 00}^{\alpha}\left(v_{\text {core }}\right)\right\rangle$, where $v_{\sigma_{\mathrm{Li}}}$ and $v_{\text {core }}$ are the respective harmonic oscillator frequencies. Defining

$$
x=\frac{v_{6 \mathrm{LL}}}{v_{\text {core }}} \approx\left(\frac{1}{6} A_{\text {core }}\right)^{t}
$$

one obtains ${ }^{78}$ )

$$
\begin{gathered}
\Omega_{00}=\left(\frac{2 \sqrt{x}}{1+x}\right)^{3} \\
\frac{\Omega_{10}}{\Omega_{00}}=\sqrt{\frac{3}{2}}\left(\frac{1-x}{1+x}\right) .
\end{gathered}
$$

Structure amplitudes will be reduced in first order by

$$
\frac{G_{N, L}(\text { finite size })}{\left.G_{N, L} \text { (zero size }\right)}=\Omega_{00}^{3} \text {. }
$$

However, part of the resulting decrease in cross section (proportional to $\Omega_{00}^{6}$ ) may be recovered by higher order terms in the expansion. Assuming the transfer of four nucleons from the same oscillator shell, Hecht ${ }^{69}$ ) obtained for SU(3) wave functions the expressions

$$
\frac{G_{N-1, L}(\text { finite size })}{G_{N, L}(\text { finite size })}=-\frac{\Omega_{10}}{\Omega_{00}} \frac{\left[\frac{3}{2}(4 q-L)(4 q+L+1)\right]^{\frac{1}{2}}}{(4 q-1)},
$$

for $(\lambda, \mu)=(Q, 0)$ and

$$
\frac{G_{N-1, L}(\text { finite size) }}{G_{N, L} \text { (finite size) }}=\frac{\Omega_{10}}{\Omega_{00}} \frac{4}{4 q-1}\left[\frac{q(q-1)\left[8(2 q-1)^{2}-L(L+1]\right.}{(4 q-2)(4 q-3)}\right]^{\frac{1}{2}} \delta_{K 0},
$$

for $(\lambda, \mu)=(Q-4,2)$. Here $Q=2 N+L=\sum q_{i}=\sum\left(2 n_{i}+l_{i}\right)$ and $q=q_{i}$.

Applying eqs. (32) $-(37)$ to the example $q_{t}=4, Q=16, A=118$ and $L=0$ one finds

$$
\begin{gathered}
\Omega_{00}=0.84, \quad \frac{\Omega_{10}}{\Omega_{00}}=-0.56, \\
\frac{G_{N, L}(\text { finite size })}{G_{N, L} \text { (zero size) }}=0.59
\end{gathered}
$$




$$
\begin{aligned}
& \frac{G_{N-1, L}(\text { finite size })}{G_{N, L}(\text { finite size })}=0.76, \quad \text { for }(\lambda, \mu)=(16,0), \\
& \frac{G_{N-1, L}(\text { finite size })}{G_{N, L}(\text { finite size })}=-0.76, \quad \text { for }(\lambda, \mu)=(12,2) .
\end{aligned}
$$

Thus, cross sections are in first order reduced by a factor

$$
\frac{\mathrm{d} \sigma / \mathrm{d} \Omega(\text { finite size })}{\mathrm{d} \sigma / \mathrm{d} \Omega(\text { zero size })}=(0.59)^{2}=0.34
$$

The second order corrections are much more difficult to estimate as they depend on the admixtures in the wave functions of four-nucleon $\mathrm{SU}(3)$ representations of lower symmetry such as $(Q-4,2)$, particularly in heavier nuclei. When no such admixtures are present one obtains

$$
\frac{\mathrm{d} \sigma / \mathrm{d} \Omega(\text { finite size) }}{\mathrm{d} \sigma / \mathrm{d} \Omega(\text { zero size })} \approx(0.59)^{2}\{1+0.76 \sqrt{0.65}\}^{2}=0.90 .
$$

It was assumed here that a reduction by one in the number of radial nodes of the cluster wave function will result in a cross section of about $65 \%$ as indicated by sample calculations. If however admixtures of lower symmetry are present, the respective contributions will at least partly cancel each other. In conclusion, a reduction in the calculated $\left(\mathrm{d},{ }^{6} \mathrm{Li}\right)$ cross section for $\mathrm{Te}$ and $\mathrm{Sn}$ targets due to finite size effects by a factor $0.6 \pm 0.25$ seems to be indicated. For more precise estimates knowledge about the admixtures is particularly important for $\alpha$-transfer in heavier nuclei as the cross sections are given by a coherent superposition of amplitudes.

Another plausible method for estimating $\alpha$-cluster size effects appears to underestimate the corrections. Eq. (27) contains two-nucleon structure amplitudes which are also reduced due to the respective overlaps for two-nucleon clusters. Glendenning $\left[\right.$ ref. $\left.\left.{ }^{69}\right)\right]$ has calculated a first order reduction for $(p, t)$ and $(t, p)$ structure amplitudes in the $\mathrm{Sn}$ region of typically $5 \%$. This correction has to be included three times in $\alpha$-cluster transfer, once each for the relative motion between the two protons and the two neutrons and between the neutron and proton pairs. This leads to a first order reduction in cross section by a factor of typically 0.7 . Second order contributions may again increase this value. These corrections appear too small, apparently due to the fact that the size of the two-neutron cluster in the triton is already relatively large and may not be too different from that in heavier nuclei. Different harmonic oscillator frequencies have to be used and the need for reliable estimates of second order corrections is again indicated. 


\section{Appendix D}

TWO-NUCLEON BCS AND PAIRING VIBRATION SPECTROSCOPIC AMPLITUDES IN THE Sn REGION

The two-nucleon parentage amplitudes

$$
\left.\left\langle\psi^{L_{v}=0} \| \chi^{L_{v}\left(J_{3} J_{4}\right.}\right)^{\dagger} \| \psi^{L_{v}}\right\rangle \quad \text { and } \quad\left\langle\psi^{\bar{L}_{\pi}=0}\left\|\chi^{L_{\pi}\left(U_{1} j_{2}\right) \dagger}\right\| \psi^{L_{\pi}}\right\rangle
$$

of eq. (27) provide the basic nuclear structure input information for the microscopic treatment of $\alpha$-particle spectroscopic amplitudes.

Two-nucleon amplitudes were taken from the work of Clement and Baranger ${ }^{18}$ ) who calculated BCS wave functions for the $\mathrm{Sn}$ isotopes from an expanded twoquasiparticle Tamm-Dankoff approximation utilizing the Tabakin interaction based on 12 neutron and 12 proton orbitals. Proton excitations were restricted to particlehole excitations. The calculated excitation energies from this work are displayed in fig. 5a (open circles).

The parentage amplitudes for zero-quasiparticle to zero-quasiparticle neutron transitions (transitions to ground states and proton pairing vibration states) are given by ${ }^{79}$ )

$$
\left\langle\psi^{L_{\nu}=0}\left\|\chi^{L_{\nu}=0(j) \dagger}\right\| \psi^{L_{\nu}=0}\right\rangle=\sqrt{j+\frac{1}{2} u_{N-2}^{j}} v_{N}^{j}
$$

where $v_{N}$ is the fullness of the target nucleus and $u_{N-2}$ the emptiness of the residual nucleus. The parentage amplitudes for zero-quasiparticle to two-quasiparticle transitions (transitions to all other excited states) are given by ${ }^{79}$ )

$$
\left\langle\psi^{\bar{L}_{v}=0} \| \chi^{L_{v}\left(j_{3} j_{4}\right) \dagger}|| \psi^{L_{v}}\right\rangle=c_{j_{3} j_{4}}\left(-\sqrt{2 J+1} v_{N}^{J_{3}} v_{N}^{j_{4}}\right),
$$

where $v_{N}^{j_{3}}$ and $v_{N}^{j_{4}}$ are the respective fullnesses of the target nuclei, $J=L_{v}$, and $c_{j_{3} j_{4}}$ is the $\left(j_{3} j_{4}\right)_{J}$ amplitude in the wave function of the residual two-quasiparticle state. Eq. (45) is valid for $j_{3}=j_{4}$ and $j_{3} \neq j_{4}$.

The two-proton amplitudes for the g.s. transitions between Te, $\mathrm{Sn}$ and $\mathrm{Cd}$ isotopes $(Z=52,50$ and 48$)$ were calculated with the ground states of the $\mathrm{Sn}$ isotopes as closed shells. The addition and removal phonon amplitudes were obtained by Vitturi et al. ${ }^{13,20}$ ) in the Tamm-Dankoff approximation by fitting the binding energy differences of the ground states in the $Z=50 \pm 2$ and $Z=50$ nuclei. The resulting wave functions are

$$
\begin{gathered}
|\mathrm{Te}\rangle=a_{1}\left|\lg _{\frac{3}{2}}^{2}\right\rangle+a_{2}\left|1 \mathrm{~d}_{\frac{3}{3}}^{2}\right\rangle+a_{3}\left|1 \mathrm{~d}_{\frac{1}{2}}^{2}\right\rangle+a_{4}\left|2 \mathrm{~s}_{\frac{1}{2}}^{2}\right\rangle+a_{5}\left|0 \mathrm{~h}_{\frac{1}{2}}^{2}\right\rangle, \\
|\mathrm{Cd}\rangle=b_{1}\left|\mathrm{~g}_{\frac{3}{2}}^{-2}\right\rangle+b_{2}\left|1 p_{\frac{1}{2}}^{-2}\right\rangle+b_{3}\left|1 p_{\frac{3}{2}}^{-2}\right\rangle,
\end{gathered}
$$

with coefficients $a_{i}$ and $b_{i}$ in good agreement with earlier work ${ }^{80-82}$ ). The coefficients $a_{i}$ and $b_{i}$ represent, of course, the required two-proton parentage amplitudes. 
If the collective coupling between proton and neutron pairs (sect. 4) is disregarded, the wave functions for the proton pairing vibration states in the $\mathrm{Sn}$ isotopes can be expressed with the usual additional/removal phonon amplitudes. The coefficients $b_{i}$ will then represent the two-proton parentage amplitudes needed for the calculations of the pickup reaction leading to these excited $0^{+}$states.

\section{References}

1) R. A. Broglia, O. Hansen and C. Riedel, Two neutron transfer reactions and the pairing model, in Advances in nuclear physics, vol. 6, ed. M. Baranger and E. Vogt (Plenum Press, New York, 1973) p. 287

2) A. Bohr, Symp. on nuclear structure, Dubna (IAEA, Vienna, 1968) p. 179

3) F. D. Becchetti, L. T. Chua, J. Jänecke and A. M. Vander Molen, Phys. Rev. Lett. 34 (1975) 225

4) F. D. Becchetti and J. Jănecke, Phys. Rev. Lett. 35 (1975) 268

5) O. Hansen. J. V. Maher, J. C. Vermeulen, L. W. Put, R. H. Siemssen and A. van der Woude, Nucl. Phys. A292 (1977) 253

6) F. L. Milder, J. Jänecke and F. D. Becchetti, Nucl. Phys. A276 (1977) 72

7) N. Stein, J. W. Sunier and C. W. Woods, Phys. Rev. Lett. 38 (1977) 587

8) M. lchimura, A. Arima, E. C. Halbert and T. Terasawa, Nucl. Phys. A204 (1973) 225

9) D. Kurath and 1. S. Towner, Nucl. Phys. A222 (1974) 1

10) R. R. Betts, in Clustering phenomena in nuclei II, ed. D. A. Goldberg et al., USERDA report ORO-4856-26 (1975) p. 458

11) R. R. Betts, Phys. Rev. C16 (1977) 1617

12) R. A. Broglia, L. Ferreira, P. D. Kunz, H. Sofia and A. Vitturi, preprint

13) A. Vitturi, L. Ferreira, P. D. Kunz, H. M. Sofia, P. F. Bortignon and R. A. Broglia, Phys. Lett. 79 B (1978) 351

14) D. Overway, F. D. Becchetti. J. Jănecke and C. E. Thorn, Bull. Am. Phys. Soc. 24 (1979) 600

15) J. Jänecke, F. D. Becchetti, D. Overway, J. D. Cossairt and R. L. Spross, Bull. Am. Phys. Soc. 24 (1979) 600

16) D. G. Fleming, M. Blann, H. W. Fulbrigh and J. A. Robbins, Nucl. Phys. A157 (1970) 1

17) H. W. Fielding, R. E. Anderson, C. D. Zafiratos, D. A. Lind, F. E. Cecil, H. H. Wieman and W. P. Alford, Nucl. Phys. A281 (1977) 389

18) D. M. Clement and E. U. Baranger, Nucl. Phys. A120 (1968) 25:

E. U. Baranger, private communication

19) R. Arvieu, E. Baranger, M. Veneroni, M. Baranger and V. Gillet, Phys. Lett. 4 (1963) 119;

R. Arvieu and E. Salusti, Nucl. Phys. 66 (1965) 305

20) A. Vitturi, private communication

21) A. Arima and F. Iachello, Phys. Rev. Lett. 35 (1975) 1069

22) A. Arima and F. lachello, Ann. of Phys. 99 (1976) 253

23) A. Arima and F. lachello, Ann. of Phys. 111 (1977) 201

24) O. Scholten, F. lachello and A. Arima, Ann. of Phys., to be published; KVI report 126 (1978)

25) J. Jänecke, F. D. Becchetti, H. Song and C. E. Thorn, Int. Conf. on nuclear structure, Tokyo, Japan (1977) p. 358;

J. Jänecke, F. D. Becchetti and C. E. Thorn, in Clustering aspects of nuclear structure (Winnipeg, 1978), American Institute of Physics Conf. Proc. Number 47, ed. W. T. H. Van Oers et al. (1978) p. 714;

F. D. Becchetti, ibid. p. 308

26) J. H. Bjerregaard, O. Hansen, O. Nathan, L. Vistisen, R. Chapman and S. Hinds, Nucl. Phys. A110 (1968) 1

27) J. H. Bjerregaard, O. Hansen, O. Nathan, R. Chapman and S. Hinds, Nucl. Phys. A131 (1969) 481

28) E. R. Flynn, J. G. Beery and A. G. Blair, Nucl. Phys. A154 (1979) 225

29) D. G. Fleming, M. Blann and H. W. Fulbright, Nucl. Phys. A163 (1971) 401

30) O. Beer, A. El Behay, P. Lopato, Y. Terrien, G. Vallois and K. K. Seth, Nucl. Phys. A147 (1970) 326 
31) W. F. Steele, P. A. Smith, J. E. Finck and G. M. Crawley, Nucl. Phys. A266 (1976) 424

32) 1975 Mass Predictions, S. Maripuu, Special Editor, Atomic Data and Nuclear Data Tables 17 (1976) 477-608

33) 1. Talmi, Nucl. Phys. A172 (1971) 1;

S. Shlomo and I. Talmi, Nucl. Phys. A198 (1972) 8 I

34) A. Arima, T. Ohtsuka, F. lachello and I. Talmi, Phys. Lett. 668 (1977) 205

35) A. Arima and F. Iachello, Phys. Rev. C16 (1977) 2085

36) T. Ohtsuka, A. Arima, F. lachello and I. Talmi, Phys. Lett. 76B (1978) 139

37) A. Arima and F. lachello, Phys. Rev. Lett. 40 (1978) 385

38) A. Bohr and B. Mottelson, Nuclear structure, vol. 11 (Benjamin, Reading, Mass., 1975) p. 646

39) J. Blomquist, Phys. Lett. 33B (1970) 541

40) E. R. Flynn and P. D. Kunz, Phys. Lett. 68B (1977) 40

41) A. H. Wapstra and K. Bos, Atomic Data and Nucl. Data Tables 19 (1977) 175

42) J. Bron, W. H. A. Wesselink, L. K. Peker, A. van Poelgeest, J. Vitzinger, H. Verheul and J. Zalmstra, J. Phys. Soc. Japan 44 (1978) Suppl. S13;

J. Bron, W. H. A. Hesselink, A. van Poelgeest, J. J. A. Zalma, M. J. Vitzinger, H. Verheul, K. Heyde, M. Waroquier, H. Vincx and P. van lsacker, Nucl. Phys. A318 (1979) 335

43) U. Garg, T. P. Sjoreen and D. P. Fossan, Phys. Rev. Lett. 40 (1978) 831 and refs. therein

44) P. D. Kunz, University of Colorado, DWUCK4 and DWUCK5, unpublished

45) A. M. Lane and R. G. Thomas, Rev. Mod. Phys. 72 (1947) 24

46) J. B. Marion and F. C. Young, Nuclear reaction analysis (American Elsevier, New York, 1968) p. 86

47) A. Bohr and B. R. Mottelson, Nuclear structure, vol. 1 (Benjamin, New York, 1970) p. 441

48) B. Buck, C. B. Dover and J. P. Vary, Phys. Rev. 11 (1975) 1803

49) K. I. Kubo and M. Hirata, Nucl. Phys. A187 (1972) 186

50) J. D. Childs, Ph.D. thesis, University of Pittsburgh (1976), unpublished;

W. W. Daehnick, private communication

51) L. T. Chua, F. D. Becchetti, J. Jänecke and F. L. Milder, Nucl. Phys. A273 (1976) 243

52) J. D. Sherman, D. L. Hendrie and M. S. Zisman, Phys. Rev. C13 (1976) 20

53) D. F. Jackson and M. Rhoades-Brown, Ann. of Phys. 105 (1977) 151

54) D. F. Jackson and M. Rhoades-Brown, Nucl. Phys. A286 (1977) 354

55) D. Kurath, Phys. Rev. C7 (1973) 1390

56) J. W. Watson, H. G. Pugh, P. G. Roos, D. A. Goldberg, R. A. J. Riddle and D. I. Bonbright, Nucl. Phys. A172 (1971) 513

57) K. Wildermuth and Y. C. Yang, A unified theory of the nucleus (Vieweg, Braunschweig, 1977) p. 64 ff

58) R. Broglia, T. Kammuri, R. Liotta, A. Winther and B. Nilsson, J. de Phys. 32, Suppl. 11-12 (1971) 151

59) H. J. Baltz and S. Kahana, Phys. Rev. Lett. 29 (1972) 1267

60) A. Roberts, Nucl. Phys. A196 (1972) 465

61) R. Broglia, R. Liotta, A. Winther, B. Nilsson and T. Kammuri, Kernforschungs analoge Jülich Internal report (1973), unpublished

62) B. F. Bayman, Phys. Rev. Lett. 32 (1974) 71

63) S. Kahana and A. J. Baltz, One- and two-nucleon transfer reactions with heavy ions, in Advances in Nuclear Physics, vol. 9, ed. M. Baranger and E. Vogt (Plenum Press, New York, 1977) p. 1

64) N. K. Glendenning, Phys. Rev. 137 (1965) B102

65) B. Bayman and A. Kallio, Phys. Rev. 156 (1967) 1121

66) N. K. Glendenning, Phys. Rev. 156 (1967) 1344

67) R. J. Ascuitto and N. K. Glendenning, Phys. Rev. 181 (1969) 1396

68) I. S. Towner and J. C. Hardy, Adv. in Phys. 18 (1969) 401

69) N. K. Glendenning, Atomic Data and Nucl. Data Tables 16 (1975) 1

70) V. A. Korolev, G. D. Alkhazov, A. A. Vorobyev, A. K. Egorov and L. M. Vasilyeva, Sov. J. Nucl. Phys. 8 (1969) 131 ;

M. C. Gupta and R. D. MacFarlaine, J. lnorg. Nucl. Chem. 32 (1970) 3425

71) F. D. Becchetti, J. Jänecke, D. Overway, J. D. Cossairt and R. L. Spross, Phys. Rev. C19 (1979) 1775

72) N. J. A. Rust, M. R. Clover, R. M. DeVries, R. Ost, R. N. Cherry and H. E. Gove, in Clustering aspects of nuclear structure and nuclear reactions (Winnipeg, 1978), American Institute of Physics Conf. Proc. Number 47, ed. W. T. H. Van Oers et al. (1978) p. 718

73) N. Hashimoto and M. Kawei, Phys. Lett. 59B (1975) 223 
74) W. W. Daehnick, M. J. Spisak, J. R. Comfort, H. Hafner and H. H. Duhm, Phys. Rev. Lett. 41 (1978) 639

75) M. A. Franey, B. F. Bayman, J. S. Lilley and W. R. Phillips, Phys. Rev. Lett. 41 (1978) 837

76) A. Saha, G. D. Jones, L. W. Put and R. H. Siemssen, preprint

77) R. S. Tickle and W. S. Gray, private communication

78) K. T. Hecht and H. Sato, in Clustering phenomena in nuclei Il, ed. D. A. Goldberg et al., USERDA report ORO-4856-26 (1975) p. 454

79) S. Yoshida, Nucl. Phys. 33 (1962) 685

80) S. Havar and R. N. Horoshko, Nucl. Phys. A183 (1972) 161

81) E. Degrieck and G. Vanden Berghe, Nucl. Phys. A231 (1974) 141

82) A. Lepine, C. Volant, M. Conjeaud, S. Harar and E. F. DaSilveira, Nucl. Phys. A289 (1977) 187

83) L. F. Hansen, F. S. Dietrich and R. P. Koopman, Phys. Rev. C18 (1978) 1582 